\title{
The heavy top: a geometric treatment
}

\author{
D Lewis $\nmid \|$, T Ratiu†ๆ, J C Simo $\neq *$ and J E Marsden $\S * *$ \\ $\dagger$ Department of Mathematics, University of California, Santa Cruz, CA 95064, USA \\ ¥ Division of Applied Mechanics, Stanford University, Stanford, CA 94305, USA \\ $\S$ Department of Mathematics, University of California, Berkeley, CA 94720, USA
}

\author{
Received 6 June 1991 \\ Accepted by J D Gíbòōn
}

\begin{abstract}
We consider the steady group motions of a rigid body with a fixed point moving in a gravitational field. For an asymmetric top, rotation about the axis of gravity is the only permissible group motion; for a Lagrange top, simultaneous rotation about the axis of gravity and spin about the axis of symmetry of the top is permissible. Our analysis of the heavy top follows the reduced energy momentum method of Simo et al , which is applicable to a wide range of conservative systems with symmetry. Steady group motions are characterized as solutions of a variational problem on the configuration space; local minima of the amended potential correspond to nonlinearly orbitally stable steady motions. The combination of a low-dimensional configuration space and a relatively large number of parameters that produce substantial qualitative changes in the dynamics makes possible a thorough, detailed analysis, which not only reproduces the classical results for this well known system, but leads to some results which we believe are new.

We determine general equilibrium and nonlinear stability conditions for steady group motions of a heavy top with a fixed point. We rederive the classical equilibrium and stability conditions for sleeping tops and precessing Lagrange tops, analyse in detail the stability of a family of steady rotations of tilted tops which bifurcate from the branch of sleeping tops parametrized by angular velocity, and classify the possible stability transitions of an arbitrary top as its angular velocity is increased. We obtain a simple, general expression for the characteristic polynomial of the linearized equations of motion and analyse the linear stability of both sleeping tops and the family of tilted top motions previously mentioned. Finally, we demonstrate the coexistence of stable branches of steadily precessing tops that bifurcate from the branch of sleeping Lagrange tops throughout the range of angular velocities for which the sleeping top is stable.
\end{abstract}

AMS classification scheme numbers: $70 \mathrm{E} 15,70 \mathrm{~K} 20,58 \mathrm{~F} 05,58 \mathrm{~F} 14,70 \mathrm{H} 30$

\section{Introduction}

The heavy top is one of the most familiar classical mechanical systems. The system, which consists of a rigid body with a fixed point moving in a gravitational field, is of sufficiently low dimension that a reasonably thorough analysis is tractable, yet rich

|| University of California Postdoctoral Fellow, partially supported by the Institute for Mathematics and its Applications and the Army Research Office.

1 Partially supported by NSF Grant DMS 9142613.

- Partially supported by AFOSR Grants 2-DJA-544 and 2-DJA-771.

** Partially supported by NSF Grant DMS-8922704 and DOE Contract DE-FGO3-88ER25064. 
enough to possess many interesting features in common with other, more complicated systems. The literature on the heavy top is substantial; Klein and Sommerfeld (1910) is an exhaustive study. Routh $(1882,1884)$ contains an elegant and thorough analysis of the stability of simple motions of heavy tops with a fixed point, as well as more complicated rigid body systems. Modern, topological treatments of the heavy top in the spirit of Smale (1970a, b) have been carried out by Iacob (1971), Katok (1972) and Tatarinov (1974); see Arnold (1989) for a summary (in English) of Tatarinov's results.

Our goal in this paper is the derivation of old, as well as new, results by means of energy-momentum techniques. Part of our goal is the demonstration of the effectiveness of these techniques in a classical setting. As shown in Simo et al (1990, 1991a, b), Lewis and Simo (1990), and Simo et al (1991), these methods are applicable to a wide variety of mechanical systems. We believe that some of our results, including a thorough bifurcation analysis of the linear stability of sleeping tops and a classification of the possible stability transitions of a broad class of steady motions, are new. The focus of our analysis is steady group motion of a heavy top. By a group motion, we mean a motion that is compatible with the symmetries of the top. For an asymmetric top, rotation about the axis of gravity is the only permissible group motion; for a Lagrange top, i.e., a heavy top whose centre of mass lies along its axis of symmetry, simultaneous rotation about the axis of gravity and spin about the axis of symmetry of the top is permissible. For these classes of motions, it is possible to characterize configurations that will maintain steady motion with a specified velocity as solutions of a variational problem. In fact, configurations that are constrained minima of the appropriate functional determine nonlinearly stable (modulo symmetries) motions. There are a number of variational analyses of steady motions of conservative systems in general and the heavy top in particular. Routh (1884) makes frequent use of variational methods; for a more recent treatment, see Maddocks (1989).

The variational technique used in the present work is the reduced energy momentum (REM) method of Simo et al (1991). This method is a synthesis of techniques developed by Riemann (1860), Routh (1882, 1884), Smale (1970a, b), Arnold (1966), Poincaré (1892) and the authors. One of the advantages of the REM method over other variational formulations is the conversion of the original constrained variational problem on phase space into an unconstrained variational problem on the configuration space. This reduced problem may be further simplified at the linear level by decomposing variations into 'rigid' and 'internal' modes with respect to which the second variation of the associated functional block diagonalizes. In consequence, the equilibrium and stability conditions can be easily derived as simple, explicit algebraic conditions.

The variational characterization of steady motion is also useful for the detection of bifurcations. When the second variation of the functional to be minimized changes signature, a new branch of configurations in steady motion may bifurcate. These branches can be detected and described in increasing detail by computing increasingly high-order variations of the functional. (See Golubitsky et al $(1985,1988)$ for a detailed discussion of singularity theory approaches to bifurcation theory.) We show here that the REM technique can greatly simplify the search for bifurcations. In particular, it sets a readily verifiable bifurcation condition on degenerate variations.

Given that a possible bifurcation of relative equilibria is signalled by a zero eigenvalue of the second variation and that the second variation is central to the stability analysis, it is natural to consider the relationship between bifurcation and stability. Typically, one expects a 'transfer of stability' at a bifurcation point: the original branch of solutions loses stability and a new stable branch of solutions appears. 
However, this need not be the case for Hamiltonian systems with symmetry. In fact, we show that under certain conditions a family of solutions parametrized by precession and spin bifurcates from a branch of sleeping Lagrange tops, while the sleeping solution branch retains stability throughout the entire range in which bifurcations occur. The Lagrange top provides an example of this phenomenon. Associated to each angular velocity for which the sleeping Lagrange top is stable, there are two one-parameter families of precessing top motions that limit to the sleeping top with the specified velocity.

\subsection{Basic notation: canonical phase space}

We consider a heavy top, i.e. a rigid body acted upon by a gravitational force, with a fixed point. Once we have specified the shape of the top and the point of the top to be fixed, the configuration space $Q$ is the set of all permissible orientations of the top. If we choose a reference configuration $\mathscr{B}$, we can identify the current configuration $\mathscr{B}_{\mathrm{t}}$ with the element $\Lambda_{\mathrm{t}}$ of the rotation group $\mathrm{SO}(3)$ taking $\mathscr{B}$ to $\mathscr{B}_{\mathrm{t}} ; \Lambda_{\mathrm{t}}$ is uniquely determined by the relation $\Lambda_{\mathrm{t}} \mathscr{B}=\mathscr{B}_{\mathrm{t}}$. Hence we set $Q=\mathrm{SO}(3)$. The tangent bundle $T Q$ consists of pairs $(\Lambda, \delta \Lambda)$, where $\Lambda \in \mathrm{SO}(3)$ and the matrix $\delta \Lambda$ satisfies the linearized orthogonality constraint

$$
\delta \Lambda^{T} \Lambda+\Lambda^{T} \delta \Lambda=\mathbf{0}
$$

i.e. $\Lambda^{T} \delta \Lambda$ is a skew-symmetric matrix:

$$
\operatorname{sym}\left[\Lambda^{T} \delta \Lambda\right]=\operatorname{sym}\left[\delta \Lambda \Lambda^{T}\right]=0
$$

Let ${ }^{-}$denote the map from $\mathbb{R}^{3}$ to the space of skew-symmetric matrices determined by

$$
\widehat{\xi} \mathbf{x}=\xi \times \mathbf{x} \quad \forall \mathbf{x} \in \mathbb{R}^{3}
$$

The condition (1.2) implies that the pair $(\Lambda, \delta \Lambda)$ is an element of $T Q$ if and only if $\Lambda \epsilon$ $\mathrm{SO}(3)$ and

$$
\Lambda \widehat{\delta}=\delta \Lambda=\widehat{\delta \theta} \Lambda
$$

for some vectors $\delta \Theta$ and $\delta \theta \in \mathbb{R}^{3}$. Thus there are two distinct identifications of the tangent bundle $T Q$ with the product $S O(3) \times \mathbb{R}^{3}$. The body variables $(\Lambda, \delta \Theta)$ correspond to the left trivialization of $T Q$; the spatial variables $(\Lambda, \delta \theta)$ correspond to the right trivialization.

The canonical phase space for the top is the cotangent bundle $T^{*} Q=T^{*} \mathrm{SO}(3)$ consisting of pairs $\left(\Lambda, \Pi_{\Lambda}\right)$ of configurations and conjugate momenta. $T^{*} Q$ also has a body and a spatial representation: $\left(\Lambda, \Pi_{\Lambda}\right) \in T^{*} Q$ if and only if there exist vectors $\Pi$ and $\pi \in \mathbb{R}^{3}$ such that

$$
\Lambda \widehat{\Pi}=\Pi_{\Lambda}=\widehat{\pi} \Lambda
$$

The covector-vector pairings in the body and spatial variables are 


$$
\left\langle\Pi_{\Lambda}, \delta \Lambda\right\rangle=\frac{1}{2} \operatorname{tr}\left((\Lambda \widehat{\Pi})^{T}(\Lambda \widehat{\delta \Theta})\right)=\Pi \cdot \delta \Theta
$$

and

$$
\left\langle\Pi_{\Lambda}, \delta \Lambda\right\rangle=\frac{1}{2} \operatorname{tr}\left((\widehat{\pi} \Lambda)^{T}(\widehat{\delta \theta} \Lambda)\right)=\pi \cdot \delta \theta .
$$

The identity

$$
\widehat{\Lambda \xi}=\Lambda \widehat{\xi} \Lambda^{T}
$$

which holds for any vector $\xi \in \mathbb{R}^{3}$ and any matrix $\Lambda \in \mathrm{SO}(3)$, implies that the body and spatial representations are related by the equalities

$$
\delta \theta=\Lambda \delta \Theta \quad \text { and } \quad \pi=\Lambda \Pi
$$

The kinetic energy of a top moving with velocity $\dot{\Lambda}$ is

$$
\frac{1}{2} \int_{\mathscr{B}} \rho_{\text {ref }}(X)(\dot{\Lambda} X) \cdot(\dot{\Lambda} X) \mathrm{d}^{3} X
$$

where $\rho_{\text {ref }}$ is the reference density. If we define the energy inner product $\langle,\rangle_{\mathbf{I}}$ on $T Q$ by

$$
\begin{aligned}
\langle U, V\rangle_{\mathbf{I}} & :=\int_{\mathbb{B}} \rho_{\text {ref }}(X)(U X) \cdot(V X) \mathrm{d}^{3} X \\
& =\operatorname{tr}\left(U^{T} V \mathbf{I}\right)
\end{aligned}
$$

where

$$
\mathbf{I}:=\int_{\mathscr{B}} \rho_{\mathrm{ref}}(X) X \otimes X \mathrm{~d}^{3} X
$$

then the kinetic energy equals $\frac{1}{2}\langle\dot{\Lambda}, \dot{\Lambda}\rangle_{\mathbf{r}}$. The gravitational potential energy is $\mathbf{g} \cdot \Lambda \mathbf{M}$, where $\mathbf{g}=\mathbf{g} \mathbf{e}_{3}$ denotes the gravity vector and $\mathbf{M}:=\int_{\mathscr{T}} \rho_{\text {ref }}(X) X \mathrm{~d}^{3} X$. If $m:=\int_{\mathscr{O}} \rho_{\text {ref }}(X) \mathrm{d}^{3} X$ is the total mass of the body, then the centre of mass of the reference body is given by $\mathbf{M} / m$. Thus $|\mathbf{M}|=m \ell$, where $\ell$ is the distance from the fixed point of the body to the centre of mass.

The inner product $\langle$,$\rangle , given by (1.11) can be expressed in terms of the pairing$ (1.6) in body representation in the following fashion. Define the reference inertia tensor $\mathbb{I}_{\text {ref }}$ by

$$
\begin{aligned}
\mathbb{I}_{\text {ref }} & =\int_{S} \rho_{\text {ref }}(X)\left(|X|^{2} \mathbf{1}_{3}-X \otimes X\right) \mathrm{d}^{3} X \\
& =(\operatorname{tr} \mathbf{I}) \mathbf{1}_{3}-\mathbf{I}
\end{aligned}
$$


where $\mathbf{I}_{3}$ denotes the three by three identity matrix. Then a straightforward computation shows that

$$
\left\langle\Lambda \widehat{\delta \Theta}_{1}, \Lambda \widehat{\delta \Theta}_{2}\right\rangle_{\mathbf{I}}=\delta \Theta_{1} \cdot \mathbb{I}_{\mathrm{ref}} \delta \Theta_{2}
$$

for any $\Lambda \in \mathrm{SO}(3)$. Note that (1.13) implies that the eigenvalues $I_{1}, I_{2}$, and $I_{3}$ of $I_{\text {ref }}$ satisfy $I_{j}=\operatorname{tr} I-\lambda_{i}$ for $i \in\{1,2,3\}$, where the $\lambda_{i}$ 's are the (positive) eigenvalues of $I$. Thus $\mathrm{I}_{j}+\mathrm{I}_{k}-\mathrm{I}_{i}=2 \lambda_{i}>0$ implies that the inequalities $\mathrm{I}_{i}<\mathrm{I}_{j}+\mathrm{I}_{k}$ hold for all $i, j$, $k \in\{1,2,3\}, j \neq k$.

The analogous expression for the inner product $\langle,\rangle_{\mathbf{I}}$ in terms of the spatial variables involves the current spatial inertia tensor $\mathbb{I}_{\Lambda}$ given by

$$
\mathbb{I}_{\Lambda}:=\Lambda \mathbb{I}_{\mathrm{ref}} \Lambda^{T}
$$

Using (1.9) and (1.14), we determine the relationship

$$
\left\langle\widehat{\delta \theta}_{1} \Lambda, \widehat{\delta \theta}_{2} \Lambda\right\rangle_{\mathbf{I}}=\left\langle\Lambda\left(\widehat{\Lambda}^{T \delta \theta_{1}}\right) \quad \Lambda\left(\widehat{\Lambda}^{T} \delta \theta_{2}\right)\right\rangle_{\mathbf{I}}=\left(\Lambda^{T} \delta \theta_{1}\right) \cdot \mathbb{I}_{\mathrm{ref}}\left(\Lambda^{T} \delta \theta_{2}\right)=\delta \theta_{1} \cdot \mathbb{I}_{\Lambda} \delta \theta_{2}
$$

for any $\Lambda \in \mathrm{SO}(3)$. Note that (1.14) shows that the metric $\langle,\rangle_{\mathbf{I}}$ on $T Q$ is left invariant, whereas $(1.16)$ shows that the metric is typically not right invariant.

\subsection{Lagrangian and Hamiltonian structures}

The Lagrangian $L$ for the heavy top is the difference between the kinetic and potential energies, i.e.,

$$
L(\Lambda, \dot{\Lambda})=\frac{1}{2}\langle\dot{\Lambda}, \dot{\Lambda}\rangle_{\mathbf{1}}-\mathbf{g} \cdot \Lambda \mathbf{M}
$$

The Legendre transformation $F L$ associated to a Lagrangian $L$, defined by

$$
F L(\Lambda, \dot{\Lambda}) \cdot(\Lambda, \delta \Lambda)=\left.\frac{\mathrm{d}}{\mathrm{d} \epsilon}\right|_{\epsilon=0} L(\Lambda, \dot{\Lambda}+\epsilon \delta \Lambda)
$$

for $\dot{\Lambda}, \delta \Lambda \in T_{\Lambda} Q$, maps velocity fields to momentum fields. In the case of the heavy top, the Legendre transformation $F L: T_{\Lambda} Q \rightarrow T_{\Lambda}^{*} Q$ at a configuration $\Lambda$ is

$$
F L(\Lambda, \Lambda \widehat{\delta \Theta})=\left(\Lambda,\langle\Lambda \widehat{\delta \Theta},\rangle_{\mathbf{l}}\right)=\left(\Lambda, \Lambda\left(\widehat{\mathbf{I}_{\mathrm{ref}} \delta \Theta}\right)\right)
$$

or, equivalently,

$$
F L(\Lambda, \widehat{\delta \theta} \Lambda)=\left(\Lambda,\langle\widehat{\partial \theta} \Lambda, \cdot\rangle_{1}\right)=\left(\Lambda,\left(\widehat{\mathbb{I}_{\Lambda} 8 \theta}\right) \Lambda\right)
$$

Formulae (1.19) and (1.20) are a direct consequence of (1.14) and (1.16) and the definition of the pairing between $T^{*} Q$ and $T Q$ given by (1.6) and (1.7). The total energy associated to the configuration-velocity pair $(\Lambda, \dot{\Lambda})$ is

$$
E(\Lambda, \dot{\Lambda})=F L(\dot{\Lambda}) \cdot \dot{\Lambda}-L(\Lambda, \dot{\Lambda})=\frac{1}{2}\langle\dot{\Lambda}, \dot{\Lambda}\rangle_{\mathbf{I}}+\mathbf{g} \cdot \Lambda \mathbf{M}
$$


To express the Lagrangian in terms of the spatial variables, define the spatial angular velocity by

$$
\widehat{\omega}:=\dot{\Lambda} \Lambda^{T} .
$$

Then (1.16) gives the expression of the Lagrangian in spatial variables as

$L(\Lambda, \omega):=L(\Lambda, \widehat{\omega} \Lambda)=\frac{1}{2}\langle\widehat{\omega} \Lambda, \widehat{\omega} \Lambda\rangle_{\mathbf{I}}-\mathbf{g} \cdot \Lambda \mathbf{M}=\frac{1}{2} \omega \cdot \mathbb{I}_{\Lambda} \omega=\mathbf{g} \cdot \mathbf{\Lambda} \mathbf{M}$

The Legendre transformation $F L: S O(3) \times \mathbb{R}^{3} \rightarrow \mathrm{SO}(3) \times \mathbb{R}^{3}$ has the spatial expression

$$
F L(\Lambda, \omega)=\left(\Lambda, \mathbb{I}_{\Lambda} \omega\right)
$$

which follows directly from (1.20). Note that we use the same letter $\underline{L}$ to denote the Lagrangian with respect to both material and spatial variables; in general, we shall use the same notation to denote a mapping with respect to material, spatial or body coordinates.

Next, we determine the Hamiltonian structure of the heavy top. The canonical phase space is the cotangent bundle $T^{*} Q$ consisting of pairs $\left(\Lambda, \Pi_{\Lambda}\right)$ of configurations and conjugate momenta. Given the Lagrangian $L$, the cotangent bundle $T^{*} Q$ is the image of the tangent bundle $T Q$ under the Legendre transformation. The Hamiltonian on the phase space $T^{*} Q$ is defined by

$$
H\left(\Lambda, \Pi_{\Lambda}\right):=E\left(\Lambda, F L^{-1}\left(\Pi_{\Lambda}\right)\right)=\mathbf{g} \cdot \Lambda \mathbf{M}+\frac{1}{2}\left\langle\Pi_{\Lambda}, \Pi_{\Lambda}\right\rangle_{\mathbf{I}^{-1}}
$$

where $\langle,\rangle_{\mathbf{1}^{-1}}$ is the metric on covectors induced by $\boldsymbol{F} L$, i.e.

$$
\left\langle\Pi_{\Lambda}, \Pi_{\Lambda}^{\prime}\right\rangle_{\mathbf{1}^{-1}}=\left\langle F L^{-1}\left(\Pi_{\Lambda}\right), F L^{-1}\left(\Pi_{\Lambda}^{\prime}\right)\right\rangle_{\mathbf{I}}
$$

In spatial representation, (1.17), (1.16) and (1.20) imply that

$$
H(\Lambda, \pi):=\mathbf{m} \cdot \mathbf{g}+\frac{1}{2} \pi \cdot \Pi_{\Lambda}^{-1} \pi
$$

where $\mathbf{m}:=\Lambda \mathbf{M}$ is the spatial representation of the mass vector. In the body representation (1.17), (1.14), and (1.19) imply that

$$
H(\Lambda, \Pi):=g \gamma \cdot \mathbf{M}+\frac{1}{2} \Pi \cdot \Pi_{\mathrm{ref}}^{-1} \Pi
$$

where $y:=\hat{\Lambda}^{T} \mathbf{e}_{3}$ is the direction of gravity in the body frame.

\subsection{Equations of motion}

Recall from section 1.2 that the spatial angular velocity $\omega$ defined by (1.22) satisfies $\widehat{\omega}=\dot{\Lambda} \Lambda^{T}$. Similarily, the body angular velocity $\Omega$ is given by

$$
\widehat{\Omega}=\Lambda^{T} \dot{\Lambda}
$$

Note that (1.9) implies that

$$
\omega=\Lambda \Omega \quad \text { and } \quad \pi=\Lambda \Pi
$$


where the body and spatial momenta

$$
\Pi=\mathbb{I}_{\mathrm{ref}} \Omega \quad \text { and } \quad \pi=\mathbb{I}_{\Lambda} \omega
$$

are given by the Legendre transformation according to (1.19) and (1.20).

The following expressions are the well-known body and spatial forms of the equations of motion; see e.g. section $(6.1)$ or Arnold $(1966,1989)$ for the derivation of these equations.

The body form of the equations of motion is

$$
\dot{\Lambda}=\Lambda \widehat{\Omega} \quad \text { and } \quad \dot{\Pi}=\Pi \times \Omega+g \gamma \times \mathbf{M} .
$$

The spatial form of the equations of motion is

$$
\dot{\Lambda}=\widehat{\omega} \Lambda \quad \text { and } \quad \dot{\pi}=\mathbf{g} \times \mathbf{m} .
$$

Note that the second equation in (1.33) is the Euler equation for a rigid body under the influence of a gravitational potential.

\subsection{Symmetries}

The symmetries of the Hamiltonian are central to the stability analysis. To obtain optimal results, it is typically necessary to work with the largest available symmetry group; failure to incorporate all symmetries can result in overly restrictive stability conditions.

We first consider the spatial symmetries of the heavy top. Material frame indifference (i.e., left $\mathrm{SO}(3)$ invariance) of the free rigid body is broken by the gravitational potential. While the kinetic energy is invariant under the full group of spatial rotations, the potential energy is invariant only under spatial rotations that preserve the gravity vector $g$. The gravitational potential is invariant not only with respect to spatial (left) rotations about $\mathrm{g}$, but also with respect to body (right) rotations about the vector passing through the centre of mass $\mathbf{M}$. If the top possesses an axis of symmetry, then the kinetic energy is right invariant under rotations about the axis of symmetry. If the top has a symmetric moment of inertia tensor and its centre of mass lies on the axis of symmetry, i.e. it is a Lagrange top, then the Hamiltonian is invariant under the group $G=S^{1} \times S^{1}$ consisting of spatial (left) rotations about the direction of gravity and body (right) rotations about the axis of symmetry.

We consider three distinct cases of steady motion, each determined by the symmetries of the top and its motion: first, the 'asymmetric' top (i.e., not a Lagrange top); second, a 'tilted' Lagrange top, for which the axis of symmetry is not parallel to the gravity vector $\mathbf{g}$; finally, the sleeping Lagrange top, for which the axis of symmetry and the gravity vector $\mathbf{g}$ are parallel. In the case of an asymmetric top, the appropriate symmetry group is $G=S^{1}$, which acts freely by spatial rotations about g. The appropriate symmetry group in the case of a Lagrange top is $G=S^{1} \times S^{1}$, which acts by rotations about $\mathbf{g}$ and the axis of symmetry. Tilted configurations have trivial isotropy, i.e. there are no elements of the group $G$ that fix the configuration. On the other hand, a sleeping Lagrange top is fixed by the one-dimensional subgroup of $G$ consisting of a rotation about $\mathbf{g}$ paired with a reverse rotation of equal magnitude about the axis of symmetry. 


\section{The asymmetric top}

We briefly outline some of the essential constructions of the reduced energy momentum (REM) method here, using the asymmetric top to illustrate the definitions, and refer the reader to Simo et al (1991) and Lewis (1991a) for the general statement of the method. A glossary of the essential constructions and a very brief summary of the method are given in the appendix. An essential feature of the analysis is the incorporation of the constant momentum constraint given by Noether's theorem. In the case of the asymmetric top, where the symmetry group is $S^{1}$, the momentum in question is simply the $\mathbf{e}_{3}$ component of the angular momentum. (In the case of the Lagrange top, which possesses a body symmetry, an additional scalar 'material momentum' is conserved.) For the asymmetric top the symmetry group $G$ is

$$
G:=\left\{\exp _{\mathrm{SO}(3)}\left[\hat{\mathbf{e}}_{3}\right]: s \in \mathbb{R}\right\} \approx S^{\mathbf{1}}
$$

with Lie algebra $g=\left\{s \hat{\mathbf{e}}_{3}: s \in \mathbb{R}\right\} \approx \mathbb{R}$. The dual $g^{*}$ to $g$ is also isomorphic to $\mathbb{R}$, with pairing given by multiplication. $G$ acts on $Q$ and $T^{*} Q$ by matrix multiplication on the left.

The infinitesimal generator associated to the $G$ action on $Q$ is

$$
\xi_{Q}(\Lambda)=\left(\Lambda, \xi \hat{\mathbf{e}}_{3} \Lambda\right)
$$

i.e., an infinitesimal rotation about the axis $\mathbf{e}_{3}$ with angular velocity $\xi$. The infinitesimal generator in the spatial representation is $\xi_{Q}(\Lambda)=\left(\Lambda, \xi \mathbf{e}_{3}\right)$. The locked inertia tensor II $(\Lambda): g \rightarrow g^{*}$ measures the momentum associated to the specified group motion; e.g., $\mathbb{I}(\hat{\Lambda}) \xi$ is the $\mathbf{e}_{3}$ component of the angular momentum of the top in orientation $\Lambda$ rotating with angular velocity $\xi$ about the $\mathbf{e}_{3}$ axis. Specifically, the locked inertia tensor associated to the $S^{1}$ action is defined by the relationship

$$
\eta \mathbb{I}(\Lambda) \xi:=\left\langle F L\left(\xi_{Q}(\Lambda)\right), \eta_{Q}(\Lambda)\right\rangle=\left(\eta \mathbf{e}_{3}\right) \cdot \mathbb{I}_{\Lambda}\left(\xi \mathbf{e}_{3}\right)=\eta \xi \mathbf{e}_{3} \cdot \mathbb{I}_{\Lambda} \mathbf{e}_{3}
$$

for all $\xi$ and $\eta \in \mathbb{R}$, where the second equality follows from (1.20) and (2.2). Hence $\mathbb{I}(\Lambda)=\mathbf{e}_{3} \cdot \mathbb{I}_{\Lambda} \mathbf{e}_{3}$.

There are two functionals that play a role in the variational problem. The first, the augmented potential, is constructed by evaluating the negative of the Lagrangian at a specified infinitesimal generator. Given $\xi \in g \approx \mathbb{R}$, the augmented potential for the asymmetric heavy top is defined by

$$
V_{\xi}(\Lambda)=-L\left(\xi_{Q}(\Lambda)\right)=\mathbf{m} \cdot \mathbf{g}-\frac{1}{2} \xi^{2} \mathbf{I}(\Lambda)
$$

A point $\Lambda_{e}$ is a critical point of $V_{\xi}$ if and only if $F L\left(\xi_{Q}\left(\Lambda_{e}\right)\right)$ is a relative equilibrium, i.e. if $\Lambda_{e}$ can be maintained in steady motion with angular velocity $\xi$. (This result is proved for general simple mechanical systems in Simo et al (1991).) However, the second variation of $V_{\xi}$ does not provide sharp stability conditions. Hence we introduce the amended potential, which is constructed by evaluating the energy at an infinitesimal generator obtained via the locked inertia tensor from a specified value of the momentum. Specifically, given $\mu \in g^{*} \approx \mathbb{R}$, the amended potential for the asymmetric heavy top is

$$
V_{\mu}(\Lambda)=E\left(\left(\mathbb{I}(\Lambda)^{-1} \mu\right)_{Q}(\Lambda)\right)=\mathbf{m} \cdot \mathbf{g}+\frac{1}{\underline{2}} \mathbb{I}(\Lambda)^{-1} \mu^{2}
$$


Critical points $\Lambda_{e}$ of $V_{\mu}$ are also relative equilibria and remain in steady rotation with the specified momentum $\mu$. Definiteness of the second variation $D^{2} V_{\mu}\left(\Lambda_{e}\right)$ on some complement to the tangent space $g \cdot \Lambda_{e}$ to the group orbit is sufficient to guarantee nonlinear stability of the motion modulo rotations about $\mathbf{e}_{3}$. The functional $V_{\mu}$ is often less convenient to work with than $V_{\xi}$; these inconveniences can be avoided to a great extent by noting that the second variation $D^{2} V_{\mu}\left(\Lambda_{e}\right)$ of the amended potential can be constructed by adding a positive semi-definite bilinear form to the second variation $D^{2} V_{\xi}\left(\Lambda_{e}\right)$ of the augmented potential. Define the locked momentum map $\mathbb{I}_{\xi}: Q \rightarrow g^{*}$ by

$$
\mathbb{I}_{\xi}(\Lambda):=\mathbb{I}(\Lambda) \xi
$$

Then the symmetric bilinear form $\mathscr{B}_{e}:=D^{2} V_{\mu}\left(\Lambda_{e}\right)$ satisfies

$\mathscr{B}_{e}(\delta \Lambda, \Delta \Lambda)=D^{2} V_{\xi}\left(\Lambda_{e}\right)(\delta \Lambda, \Delta \Lambda)+\left(D \mathbb{I}_{\xi}\left(\Lambda_{e}\right) \cdot \delta \Lambda\right) \cdot \mathbb{I}\left(\Lambda_{e}\right)^{-1}\left(D \mathbb{I}_{\xi}\left(\Lambda_{e}\right) \cdot \Delta \Lambda\right)$

for all variations $\delta \Lambda, \Delta \Lambda \in T_{\Lambda_{e}} Q$. (See Simo et al (1991) for the general statement of this result for simple mechanical systems.)

\subsection{Relative equilibria-the first variation}

We use the augmented potential $V_{\xi}$ to determine relative equilibria. Using standard vector product identities, the first variation of the locked inertia tensor is computed as follows:

$$
\begin{aligned}
D \mathbb{I}(\Lambda) \cdot \delta \Lambda & =\mathbf{e}_{3} \cdot\left(\delta \Lambda \mathbb{I}_{\mathrm{ref}} \Lambda^{T}+\Lambda \mathbb{I}_{\mathrm{ref}} \delta \Lambda^{T}\right) \mathbf{e}_{3} \\
& =\mathbf{e}_{3} \cdot\left(\widehat{\delta \theta} \mathbb{I}_{\Lambda}-\mathbb{I}_{\Lambda} \widehat{\delta \theta}\right) \mathbf{e}_{3} \\
& =\mathbf{e}_{3} \cdot\left(\delta \theta \times \mathbb{I}_{\Lambda} \mathbf{e}_{3}\right)-\mathbb{I}_{\Lambda} \mathbf{e}_{3} \cdot\left(\delta \theta \times \mathbf{e}_{3}\right) \\
& =2 \delta \theta \cdot\left(\left(\mathbf{I}_{\Lambda} \mathbf{e}_{3}\right) \times \mathbf{e}_{3}\right)
\end{aligned}
$$

where $\hat{\delta \theta}=\delta \Lambda \Lambda^{T}$. The first variation of the gravitational potential in the direction of the variation $\delta \Lambda$ is

$$
g \mathbf{e}_{3} \cdot \delta \Lambda \mathbf{M}=g \delta \theta \cdot\left(\mathbf{m} \times \mathbf{e}_{3}\right) .
$$

Thus relative equilibria are determined by the condition

$$
0=D V_{\xi}\left(\Lambda_{e}\right) \cdot \delta \Lambda=\delta \theta \cdot\left(\left(g \mathbf{m}_{e}-\xi^{2} \mathbb{I}_{\Lambda_{e}} \mathbf{e}_{3}\right) \times \mathbf{e}_{3}\right)
$$

for all $\delta \Lambda \in T_{\Lambda_{e}} Q$, i.e.,

$$
\left(g \mathbf{m}_{e}-\xi^{2} \mathbf{I}_{\Lambda_{e}} \mathbf{e}_{3}\right) \times \mathbf{e}_{3}=\mathbf{0} .
$$

The relative equilibria determined by (2.11) are traditionally referred to as the 'axes of Staude' (see, e.g., Maddocks (1989)). When performing the stability analysis, we shall find it convenient to express the equilibrium condition in the form

$$
g \mathbf{m}_{e}-\xi^{2} \mathbf{I I}_{\Lambda_{e}} \mathbf{e}_{3}=\Gamma_{e} \mathbf{e}_{3}
$$

where

$$
\Gamma_{e}:=\mathbf{m}_{e} \cdot \mathbf{g}-\xi^{2} \Pi\left(\Lambda_{e}\right)=\left(g \mathbf{m}_{e}-\xi^{2} \mathbb{I}_{\Lambda_{e}} \mathbf{e}_{3}\right) \cdot \mathbf{e}_{3}
$$




\subsection{Stability-the second variation}

Let $\delta \Lambda=\widehat{\delta \theta} \Lambda_{e}$ and $\Delta \Lambda=\widehat{\Delta \theta} \Lambda_{e}$ denote two elements of $T_{\Lambda_{e}} Q$. The second variation of the augmented potential is

$$
\begin{aligned}
D^{2} V_{\xi}\left(\Lambda_{e}\right)(\delta \Lambda, \Delta \Lambda)=\left(\mathbf{e}_{3} \times \delta \theta\right) \cdot\left(\Delta \theta \times g \mathbf{m}_{e}-\xi^{2}\left(\Delta \theta \times \mathbb{I}_{\Lambda_{e}} \mathbf{e}_{3}-\mathbb{I}_{\Lambda_{e}}\left(\Delta \theta \times \mathbf{e}_{3}\right)\right)\right) \\
=-\xi^{2}\left(\mathbf{e}_{3} \times \delta \theta\right) \cdot \mathbb{I}_{\Lambda_{e}}\left(\mathbf{e}_{3} \times \Delta \theta\right) \\
\quad+\left(\mathbf{e}_{3} \times \delta \theta\right) \cdot \Delta \theta \times\left(g \mathbf{m}_{e}-\xi^{2} \mathbb{I}_{\Lambda_{e}} \mathbf{e}_{3}\right) \\
=-\left(\mathbf{e}_{3} \times \delta \theta\right) \cdot\left(\xi^{2} \mathbb{I}_{\Lambda_{e}}+\Gamma_{e} \mathbf{1}_{3}\right)\left(\mathbf{e}_{3} \times \Delta \theta\right) .
\end{aligned}
$$

The momentum correction term in (2.7) is given by

$$
\begin{aligned}
\left(D \mathbb{I}_{\xi}\left(\Lambda_{e}\right) \cdot \delta \Lambda\right) \cdot \mathbb{I}\left(\Lambda_{e}\right)^{-1}\left(D \mathbb{I}_{\xi}\left(\Lambda_{e}\right) \cdot \Delta \Lambda\right) & \\
\quad= & \frac{\xi^{2}}{\mathbb{I}\left(\Lambda_{e}\right)}\left(D \mathbb{I}\left(\Lambda_{e}\right) \cdot \delta \Lambda\right)\left(D \mathbb{I}\left(\Lambda_{e}\right) \cdot \Delta \Lambda\right) \\
& =\frac{4 \xi^{2}}{\mathbb{I}\left(\Lambda_{e}\right)}\left(\mathbf{e}_{3} \times \delta \theta\right) \cdot\left(\mathbb{I}_{\Lambda_{e}} \mathbf{e}_{3} \otimes \mathbb{I}_{\Lambda_{e}} \mathbf{e}_{3}\right)\left(\mathbf{e}_{3} \times \Delta \theta\right) .
\end{aligned}
$$

Substitution of expressions (2.14) and (2.15) into (2.7) yields

$\mathscr{B}_{e}(\delta \Lambda, \Delta \Lambda)=\left(\mathbf{e}_{3} \times \delta \theta\right) \cdot\left(\frac{4 \xi^{2}}{\mathbb{I}\left(\Lambda_{e}\right)} \mathbb{I}_{\Lambda_{e}} \mathbf{e}_{3} \otimes \mathbb{I}_{\Lambda_{e}} \mathbf{e}_{3}-\xi^{2} \mathbb{I}_{\Lambda_{e}}-\Gamma_{e} \mathbf{1}_{3}\right)\left(\mathbf{e}_{3} \times \Delta \theta\right)$.

The equilibrium is orbitally nonlinearly stable if $\mathscr{B}_{e}$ is positive semi-definite, with kernel spanned by $\hat{\mathbf{e}}_{3} \Lambda_{e}$, i.e., if the restriction $\widetilde{\mathscr{B}}_{e}$ of the form

$$
\frac{4 \xi^{2}}{\mathbb{I}\left(\Lambda_{e}\right)} \mathbb{I}_{\Lambda_{e}} \mathbf{e}_{3} \otimes \mathbb{I}_{\Lambda_{e}} \mathbf{e}_{3}-\xi^{2} \mathbf{I}_{\Lambda_{e}}-\Gamma_{e} \mathbf{1}_{3}
$$

to $\operatorname{span}\left\{\mathbf{e}_{1}, \mathbf{e}_{2}\right\}$ is positive definite. The equilibrium condition (2.12) implies that the restriction of (2.17) to $\operatorname{span}\left\{\mathbf{e}_{1}, \mathbf{e}_{2}\right\}$ equals

$$
\frac{4 g^{2}}{\xi^{2} \mathbb{I}\left(\Lambda_{e}\right)} \mathbf{m}_{e} \otimes \mathbf{m}_{e}-\xi^{2} \mathbb{I}_{\Lambda_{e}}-\Gamma_{e} \mathbf{1}_{3}
$$

The stability conditions can easily be formulated in terms of the body vectors $\mathbf{M}$ and $\gamma$. The matrix (2.17) can be written in the form

$$
\Lambda_{e}\left(\frac{4 \xi^{2}}{\left(\gamma_{e} \cdot \mathbb{I}_{\mathrm{ref}} \gamma_{e}\right)} \mathbb{I}_{\mathrm{ref}} \gamma_{e} \otimes \mathbb{I}_{\mathrm{ref}} \gamma_{e}-\xi^{2} \mathbb{I}_{\mathrm{ref}}-\Gamma_{e} \mathbf{1}_{\mathfrak{3}}\right) \Lambda_{e}^{T}
$$

Hence $\widetilde{\mathscr{B}}_{e}$ is positive definite if and only if the restriction of

$$
\frac{4 \xi^{2}}{I_{\gamma_{e}}} \mathbb{I}_{\mathrm{ref}} \gamma_{e} \otimes \mathbb{I}_{\mathrm{ref}} \gamma_{e}-\xi^{2} \mathbb{I}_{\mathrm{ref}}-\Gamma_{e} \mathbf{1}_{3}
$$

or, equivalently,

$$
\frac{4 g^{2}}{\xi^{2} I_{\gamma_{e}}} \mathbf{M} \otimes \mathbf{M}-\xi^{2} I_{\mathrm{ref}}-\Gamma_{e} \mathbf{1}_{3}
$$


to the plane orthogonal to $\gamma_{e}$ is positive definite.

In many cases, the test for positive definiteness of $\widetilde{\mathscr{B}}_{e}$ is most easily implemented by testing for positivity of the trace and determinant of $\widetilde{\mathscr{B}}_{e}$. Hence, we derive below some simple expressions for these invariants. If we define

$$
\mathscr{C}_{e}:=\left.\xi^{2} \mathrm{I}_{\Lambda_{e}}\right|_{\mathbf{e}_{1}-\mathbf{e}_{2}}+\Gamma_{e} \mathbf{1}_{2}
$$

then

$$
\widetilde{\mathscr{B}}_{e}=\frac{4 \xi^{2}}{\mathbb{I}\left(\Lambda_{e}\right)}\left(\mathbb{I}_{\Lambda_{e}} \mathbf{e}_{3}-\mathbb{I}\left(\Lambda_{e}\right) \mathbf{e}_{3}\right) \otimes\left(\mathbb{I}_{\Lambda_{e}} \mathbf{e}_{3}-\mathbf{I}\left(\Lambda_{e}\right) \mathbf{e}_{3}\right)-\mathscr{C}_{e}
$$

Using the identities

$$
\operatorname{tr}(\mathbf{x} \otimes \mathbf{x}-A)=|\mathbf{x}|^{2}-\operatorname{tr} A
$$

and

$$
\operatorname{det}(\mathbf{x} \otimes \mathbf{x}-A)=\operatorname{det} A-\left(\mathbf{e}_{3} \times \mathbf{x}\right) \cdot A\left(\mathbf{e}_{3} \times \mathbf{x}\right)
$$

for all two by two symmetric matrices $A$ and all $\mathbf{x} \in \operatorname{span}\left\{\mathbf{e}_{1}, \mathbf{e}_{2}\right\}$, we see that

$$
\operatorname{tr} \widetilde{\mathscr{B}}_{e}=\frac{4 \xi^{2}}{\mathbb{I}\left(\Lambda_{e}\right)}\left|\mathbf{e}_{3} \times \mathbb{I}_{\Lambda_{e}} \mathbf{e}_{3}\right|^{2}-\operatorname{tr} \mathscr{C}_{e}
$$

and

$$
\operatorname{det} \widetilde{\mathscr{B}}_{e}=\operatorname{det} \mathscr{C}_{e}-\frac{4 \xi^{2}}{\mathbb{I}\left(\Lambda_{e}\right)}\left(\mathbf{e}_{3} \times \mathbb{I}_{\Lambda_{e}} \mathbf{e}_{3}\right) \cdot \mathscr{C}_{e}\left(\mathbf{e}_{3} \times \mathbb{I}_{\Lambda_{e}} \mathbf{e}_{3}\right)
$$

Hence $\widetilde{\mathscr{B}}_{e}$ is positive definite if and only if

$$
\frac{4 \xi^{2}}{\mathbb{I}\left(\Lambda_{e}\right)}\left|\mathbf{e}_{3} \times \mathbb{I}_{\Lambda_{e}} \mathbf{e}_{3}\right|^{2}>\operatorname{tr} \mathscr{C}_{e}
$$

and

$$
\operatorname{det} \mathscr{C}_{e}>\frac{4 \xi^{2}}{\mathbb{I}\left(\Lambda_{e}\right)}\left(\mathbf{e}_{3} \times \mathbb{I}_{\Lambda_{e}} \mathbf{e}_{3}\right) \cdot \mathscr{C}_{e}\left(\mathbf{e}_{3} \times \mathbf{I}_{\Lambda_{e}} \mathbf{e}_{3}\right)
$$

Making use of the equilibrium condition (2.11), i.e.

$$
\mathbf{e}_{3} \times \mathbb{I}_{\Lambda_{e}} \mathbf{e}_{3}=\frac{g}{\xi^{2}} \mathbf{e}_{3} \times \mathbf{m}_{e}
$$

we can express the stability conditions in the form

$$
\frac{4 g^{2}}{\xi^{2} \mathbb{I}\left(\Lambda_{e}\right)}\left|\mathbf{e}_{3} \times \mathbf{m}_{e}\right|^{2}>\operatorname{tr} \mathscr{C}_{e}
$$


and

$$
\operatorname{det} \mathscr{C}_{e}>\frac{4 g^{2}}{\xi^{2} \Pi\left(\Lambda_{e}\right)}\left(\mathbf{e}_{3} \times \mathbf{m}_{e}\right) \cdot \mathscr{C}_{e}\left(\mathbf{e}_{3} \times \mathbf{m}_{e}\right)
$$

In summary, we have derived the following equilibrium and stability conditions:

An asymmetric top with spatial inertia tensor $\mathbb{I}_{\Lambda_{e}}$ and mass vector $\mathbf{m}_{e}$ can be maintained in steady rotation about the axis of gravity $\mathbf{g}=\mathrm{ge}_{3}$ with angular velocity $\xi$ if and only if

$$
\mathbf{g} \mathbf{m}_{e}-\xi^{2} \mathbf{I}_{\Lambda_{e}} \mathbf{e}_{3}=\Gamma_{e} \mathbf{e}_{3}
$$

equivalently, if and only if

$$
g \mathbf{M}-\xi^{2} \Pi_{\mathrm{ref}} \gamma_{e}=\Gamma_{e} \gamma_{e}
$$

where $\gamma_{e}:=\Lambda_{e}^{T} \mathbf{e}_{3}$ is the direction of gravity with respect to the body frame and

$$
\Gamma_{e}=\mathbf{m}_{e} \cdot \mathbf{g}-\xi^{2} \mathbb{I}\left(\Lambda_{e}\right)=g \gamma_{e} \cdot \mathbf{M}-\xi^{2} \gamma_{e} \cdot \mathbb{I}_{\mathrm{ref}} \gamma_{e} .
$$

This steady rotation is nonlinearly orbitally stable if the restriction $\widetilde{\mathscr{B}}_{e}$ of the symmetric matrix

$$
\frac{4 \xi^{2}}{\mathbf{I}\left(\Lambda_{e}\right)} \mathbf{I}_{\Lambda_{e}} \mathbf{e}_{3} \otimes \mathbb{I}_{\Lambda_{e}} \mathbf{e}_{3}-\xi^{2} \mathbf{I}_{\Lambda_{e}}-\Gamma_{e} \mathbf{1}_{3}
$$

to the $\hat{\mathbf{e}}_{1}-\mathbf{e}_{2}$ plane is positive definite.

\subsection{Stability of special classes of relative equilibria}

In this section, we explicitly parametrize some special classes of steady motions of heavy tops that are characterized by conditions on the position of the axis of gravity relative to the eigenvectors of the inertia tensor and present the equilibrium and stability conditions in terms of these parameters. The Hamiltonian is determined by the eigenvalues of the reference inertia tensor $\mathbb{I}_{\text {ref }}$, the mass of the top, the coordinates of the centre of mass with respect to the principal axes of the reference inertia tensor, and the strength of gravity. The quantities characterizing the steady motion of the top are the angle of the cone traced out by the motion of the vector from the fixed point to the centre of mass, and the angular velocity $\xi$.

We first apply the stability criteria to a sleeping asymmetric top, i.e., a top for which the gravity vector $\mathbf{g}=\mathrm{ge}_{3}$ is an eigenvector of the equilibrium inertia tensor $\mathbb{I}_{\Lambda_{e}}$. Taking the remaining eigenvectors of the inertia tensor as our basis vectors, we may assume that $\mathbb{I}_{\Lambda_{\varepsilon}}=\operatorname{diag}\left[\mathrm{I}_{1}, \mathrm{I}_{2}, \mathrm{I}_{3}\right]$ for some positive constants $\mathrm{I}_{i}$ satisfying $\mathrm{I}_{1}>\mathrm{I}_{2}$. The equilibrium equations (2.12) imply that $\mathbf{m}_{e}= \pm m \ell \mathbf{e}_{3}$. A positive sign implies that the top is upright; a negative sign implies that the top is hanging straight down. Conversely, if $\mathbf{m}_{e}$ and $\mathbf{e}_{3}$ are proportional, then $\mathbf{e}_{3}$ must be an eigenvector of $\mathbb{I}_{\Lambda_{e}}$ in the case of steady motion, i.e., sleeping tops are characterized by the condition that the mass vector $\mathbf{m}_{e}$ is parallel to the gravity vector $\mathbf{g}$. 
A sleeping top $\Lambda_{e}$ can be kept in steady rotation at any angular velocity, i.e. $D V_{\xi}\left(\Lambda_{e}\right)=\mathbf{0}$ for any $\xi \in \mathbb{R}$. The stability matrix $\widetilde{\mathscr{B}}_{e}$ for an upright top is given by

$$
\widetilde{\mathscr{B}}_{e}=\operatorname{diag}\left[\xi^{2}\left(\mathrm{I}_{3}-\mathrm{I}_{1}\right)-m g \ell, \xi^{2}\left(\mathrm{I}_{3}-\mathrm{I}_{2}\right)-m g \ell\right] .
$$

Hence an upright sleeping top, i.e. a top for which $\ell$ is positive, rotating at angular velocity $\xi$ is nonlinearly stable modulo rotations about $\mathbf{e}_{3}$ if it is in rotation about the axis of maximal inertia, i.e. if $I_{3}>I_{1}>I_{2}$, and

$$
\xi^{2}>\frac{m g \ell}{\mathrm{I}_{3}-\mathrm{I}_{1}} .
$$

A hanging sleeping top has stability matrix $\widetilde{\mathscr{B}}_{e}=\operatorname{diag}\left[\xi^{2}\left(\mathrm{I}_{3}-\mathrm{I}_{1}\right)+m g \ell, \xi^{2}\left(\mathrm{I}_{3}-\mathrm{I}_{2}\right)+m g \ell\right]$. Hence a hanging top in rotation about the axis of maximal inertia is nonlinearly stable at any velocity. A hanging sleeping top for which either $I_{1}>I_{3}>I_{2}$ or $I_{1}>I_{2}>I_{3}$ is stable for angular velocities $\xi$ satisfying

$$
\xi^{2}<\frac{m g \ell}{\mathrm{I}_{1}-\mathrm{I}_{3}}
$$

These stability conditions for sleeping tops can be found in Routh (1884, section 211).

A tilted top is a top for which the gravity vector $\mathbf{g}=g_{3}$ is not an eigenvector of the inertia tensor $\mathbb{I}_{\Lambda_{e}}$. If it can maintain a steady rotation about the axis of gravity without spin, then it is a relative equilibrium. There is a special family of tilted relative equilibria that generalizes the family of sleeping tops analysed above; specifically, we consider tops for which the axis of gravity lies in a plane determined by two of the principal axes of the inertia tensor. To simplify the general calculations carried out in sections 2.1 and 2.2 , we shall construct a coordinate system tailored to the candidate relative equilibrium. By assumption, there is an eigenvector $\mathbf{e}_{1}$ of $\mathbb{I}_{\Lambda_{e}}$ such that $\mathbf{e}_{1} \cdot \mathbf{g}=0$. Hence we can set $\mathbf{e}_{2}:=\mathbf{e}_{3} \times \mathbf{e}_{1}$. In this coordinate system, the spatial inertia tensor $\mathbb{I}_{\Lambda_{e}}$ is given by

$$
\mathrm{I}_{\Lambda_{e}}=R_{\theta} \operatorname{diag}\left[\mathrm{I}_{1}, \mathrm{I}_{2}, \mathrm{I}_{3}\right] R_{\theta}^{T}=\left(\begin{array}{ccc}
\mathrm{I}_{1} & 0 & 0 \\
0 & \mathrm{I}_{2} \cos ^{2} \theta+\mathrm{I}_{3} \sin ^{2} \theta & \left(\mathrm{I}_{2}-\mathrm{I}_{3}\right) \cos \theta \sin \theta \\
0 & \left(\mathrm{I}_{2}-\mathrm{I}_{3}\right) \cos \theta \sin \theta & \mathrm{I}_{2} \sin ^{2} \theta+\mathrm{I}_{3} \cos ^{2} \theta
\end{array}\right)
$$

where

$$
R_{\theta}:=\left(\begin{array}{ccc}
1 & 0 & 0 \\
0 & \cos \theta & -\sin \theta \\
0 & \sin \theta & \cos \theta
\end{array}\right)
$$

for some angle $\theta \neq 0, \pi$. Note that the stability of a specific top is determined by the spatial equilibrium values $\mathbb{I}_{\Lambda_{e}}$ and $\mathbf{m}_{e}$, as well as the equilibrium velocity $\xi$; the stability conditions are independent of the specific choice of a reference orientation of the top. Hence we are free to make any convenient choice of principal axes of the body frame. We set $\mathbb{I}_{\text {ref }}=\operatorname{diag}\left[\mathrm{I}_{1}, \mathrm{I}_{2}, \mathrm{I}_{3}\right]$ and $\Lambda_{e}=R_{\theta}$.

We now determine the equilibrium mass vector $\mathbf{m}_{e}=\Lambda_{e} \mathbf{M}$. Since $\Lambda_{e}$ is a rotation about $\mathbf{e}_{1}$ and hence fixes $\mathbf{e}_{1},(2.36)$ and the equilibrium condition (2.12) imply that

$$
g \mathbf{M} \cdot \mathbf{e}_{1}=g \mathbf{m}_{e} \cdot\left(\Lambda_{e} \mathbf{e}_{1}\right)=g \mathbf{m}_{e} \cdot \mathbf{e}_{1}=\xi^{2} \mathbf{e}_{1} \cdot \mathbb{I}_{\Lambda_{e}} \mathbf{e}_{3}=0
$$


Hence

$$
\mathbf{M}=m \ell(0, \sin \phi, \cos \phi)
$$

and

$$
\mathbf{m}_{e}=\Lambda_{e} \mathbf{M}=m \ell R_{(\theta-\phi)} \mathbf{e}_{3}=(0, m \ell \sin (\phi-\theta), m \ell \cos (\phi-\theta))
$$

for some angle $\phi$. The $\mathbf{e}_{2}$ component of (2.12) yields the equilibrium condition

$$
m g \ell \sin (\phi-\theta)=\xi^{2}\left(\mathrm{I}_{2}-\mathrm{I}_{3}\right) \cos \theta \sin \theta .
$$

Since we assume that the top is not a sleeping top, the right hand side of $(2.41)$ is non-zero. Thus, the equilibrium velocity $\xi$ satisfies

$$
\xi^{2}=\frac{2 m g \ell \sin (\theta-\phi)}{\left(\mathrm{I}_{3}-\mathrm{I}_{2}\right) \sin 2 \theta}
$$

The stability conditions for relative equilibria tilted about $\mathbf{e}_{1}$ are obtained by substituting (2.36) and (2.42) into (2.16). The equilibrium value of the locked inertia tensor is

$\mathbb{I}\left(\Lambda_{e}\right)=\mathbf{e}_{3} \cdot \mathbb{I}_{\Lambda_{e}} \mathbf{e}_{3}=\mathrm{I}_{2} \sin ^{2} \theta+\mathrm{I}_{3} \cos ^{2} \theta=\frac{1}{2}\left(\mathrm{I}_{2}+\mathrm{I}_{3}+\left(\mathrm{I}_{3}-\mathrm{I}_{2}\right) \cos 2 \theta\right)$.

Hence, using (2.42), we see that

$$
\begin{aligned}
\Gamma_{e}=g \mathbf{m}_{e} \cdot & \mathbf{e}_{3}-\xi^{2} \mathbb{I}\left(\Lambda_{e}\right) \\
= & \frac{1}{2} \xi^{2}\left(\left(\mathrm{I}_{3}-\mathrm{I}_{2}\right) \sin 2 \theta \cot (\theta-\phi)-\left(\mathrm{I}_{2}+\mathrm{I}_{3}+\left(\mathrm{I}_{3}-\mathrm{I}_{2}\right) \cos 2 \theta\right)\right) \\
= & -\frac{1}{2} \xi^{2}\left(\mathrm{I}_{2}+\mathrm{I}_{3}-\left(\mathrm{I}_{3}-\mathrm{I}_{2}\right) \rho(\theta, \phi)\right)
\end{aligned}
$$

where

$$
\rho(\theta, \phi):=\frac{\sin (\theta+\phi)}{\sin (\theta-\phi)} .
$$

The stability matrix is $\widetilde{\mathscr{B}}_{e}=\operatorname{diag}\left[b_{1}, b_{2}\right]$, where

$$
b_{1}:=-\xi^{2} \mathbf{e}_{1} \cdot \mathrm{I}_{\Lambda_{e}} \mathbf{e}_{1}-\Gamma_{e}=\frac{1}{2} \xi^{2}\left(\mathrm{I}_{2}+\mathrm{I}_{3}-2 \mathrm{I}_{1}-\left(\mathrm{I}_{3}-\mathrm{I}_{2}\right) \rho(\theta, \phi)\right)
$$

and

$$
\begin{aligned}
& \left.b_{2}:=\frac{4 \xi^{2}}{\mathbf{e}_{3}} \cdot \frac{\mathbb{I}_{\Lambda_{e}} \mathbf{e}_{3}}{\mathbf{e}_{2}} \cdot \mathbb{I}_{\Lambda_{e}} \mathbf{e}_{3}\right)^{2}-\xi^{2} \mathbf{e}_{2} \cdot \mathbb{I}_{\Lambda_{e}} \mathbf{e}_{2}-\Gamma_{e} \\
& =\frac{1}{2} \xi^{2}\left(I_{3}-I_{2}\right)\left(\frac{4\left(I_{3}-I_{2}\right) \sin ^{2} 2 \theta}{I_{2}+I_{3}+\left(I_{3}-I_{2}\right) \cos 2 \theta}+\cos 2 \theta-\rho(\theta, \phi)\right) \text {. }
\end{aligned}
$$

The steady rotation of the top is stable if $b_{1}$ and $b_{2}$ are both positive. Hence, if we define

$\tau_{1}:=\frac{I_{2}+I_{3}-2 I_{1}}{I_{3}-I_{2}} \quad$ and $\quad \tau_{2}:=\frac{4\left(I_{3}-I_{2}\right) \sin ^{2} 2 \theta}{I_{2}+I_{3}+\left(I_{3}-I_{2}\right) \cos 2 \theta}+\cos 2 \theta$ 
then the steady rotation is stable if

$$
\mathrm{I}_{3}>\mathrm{I}_{2} \quad \text { and } \quad \min \left\{\tau_{1}, \tau_{2}\right\}>\rho(\theta, \phi)
$$

or if

$$
\mathrm{I}_{2}>\mathrm{I}_{3} \quad \text { and } \quad \rho(\theta, \phi)>\max \left\{\tau_{1}, \tau_{2}\right\} .
$$

If $\phi=0$, i.e., if the reference centre of mass $\mathbf{M}$ lies on the principal axis of $\mathbb{I}_{\text {ref }}$ with eigenvalue $\mathrm{I}_{3}$ (and hence the spatial centre of mass $\mathbf{m}_{e}$ lies along the principal axis of $\mathbb{I}_{\Lambda_{e}}$ with eigenvalue $\mathbb{I}\left(\Lambda_{e}\right)$ ), then the expressions appearing above are substantially simplified. The equation $(2.42)$ for the equilibrium velocity then takes the form

$$
\xi^{2}=\frac{m g \ell}{\left(\mathrm{I}_{3}-\mathrm{I}_{2}\right) \cos \theta}
$$

Hence, if $\mathrm{I}_{3}>\mathrm{I}_{2}$, then tilted relative equilibria with centre of mass along a principal axis exist only for $\pi / 2>\theta>-\pi / 2$, i.e. the centre of mass is above the fixed point of the top. On the other hand, if $\mathrm{I}_{2}>\mathrm{I}_{3}$, then the centre of mass lies below the fixed point, with $3 \pi / 2>\theta>\pi / 2$. The diagonal entries of the stability matrix $\widetilde{B_{B}}{ }_{e}$ are given by $b_{1}=\xi^{2}\left(\mathrm{I}_{2}-\mathrm{I}_{1}\right)$ and

$$
b_{2}=\frac{\xi^{2}\left(I_{3}-I_{2}\right)}{I_{2}+I_{3} \cot ^{2} \theta}\left[3\left(I_{3}-I_{2}\right) \cos ^{2} \theta-I_{2}\right] .
$$

The condition $b_{1}>0$ yields $\mathrm{I}_{2}>\mathrm{I}_{1}$. To impose the condition $b_{2}>0$, we distinguish two cases. If $I_{3}>I_{2}>I_{1}$, then $b_{2}>0$ implies that $3\left(I_{3}-I_{2}\right) \cos ^{2} \theta>I_{2}$, which implies that $\mathrm{I}_{2}>3 \mathrm{I}_{3} / 4$ and

$$
\cos ^{2} \theta>\frac{I_{2}}{3\left(I_{3}-I_{2}\right)} .
$$

If $I_{2}>I_{1}$ and $I_{2}>I_{3}$, then both $b_{1}$ and $b_{2}$ are positive for any angle $\theta$, i.e. this steady motion of the top is stable.

Remark: The Kovalevskaya top: A special class of Kovalevskaya tops is determined by the conditions $\mathrm{I}_{1}=\mathrm{I}_{3}=2 \mathrm{I}_{2}$ and $\phi=0$. If $\theta=0$, then $\widetilde{\mathscr{B}}_{\ell}=\operatorname{diag}\left[-m g \ell, \xi^{2} \mathrm{I}_{2}-m g \ell\right]$; hence the top is formally unstable. If $\theta=\pi$, then $\widetilde{\mathscr{B}}_{e}=\operatorname{diag}\left[m g \ell, \xi^{2} \mathrm{I}_{2}+m g \ell\right]$ is positive definite; hence the hanging Kovalevskaya top is stable for all velocities. Finally, if $\cos ^{2} \theta \neq 1$, then $b_{1}=\xi^{2}\left(\mathrm{I}_{2}-\mathrm{I}_{1}\right)=-\xi^{2} \mathrm{I}_{2}<0$ implies that the motion is formally unstable. These results are given in Levi-Civita (1901). We shall show in section 4 that for $\theta$ satisfying $0<\cos ^{2} \theta<1 / 3$, these relative equilibria are linearly stable, even though they fail to be formally stable.

We summarize our results as follows:

Consider an asymmetric top, whose inertia tensor has eigenvalues $\mathrm{I}_{1}, \mathrm{I}_{2}$, and $\mathrm{I}_{3}$, and an eigenvector $\mathbf{e}_{1}$ (with eigenvalue $\mathrm{I}_{1}$ ) of $\mathrm{I}_{\Lambda_{e}}$ satisfying $\mathbf{e}_{1} \cdot \mathbf{g}=\mathbf{e}_{1} \cdot \mathbf{m}_{e}=0$. Let $\theta$ denote the angle in the plane orthogonal to $\mathbf{e}_{1}$ between the axis of gravity $\mathbf{g}$ and the eigenvector of 
$\mathbb{I}_{\Lambda_{e}}$ with eigenvalue $\mathrm{I}_{3}$ and let $\phi$ denote the angle in the same plane between $\mathbf{m}_{e}$ and this eigenvector, i.e. $\mathbf{m}_{e} \cdot \mathbf{g}=m g \ell \cos (\phi-\theta)$. The top can be maintained in steady rotation about the axis of gravity $\mathbf{e}_{3}$ with angular velocity $\xi$ if either

$$
\theta=0, \pi \quad \text { or } \quad \xi^{2}=\frac{2 m g \ell \sin (\theta-\phi)}{\left(\mathrm{I}_{3}-\mathrm{I}_{2}\right) \sin 2 \theta} .
$$

The nonlinear orbital stability conditions for steady motion of the asymmetric top are (i) $\theta=\phi=0$. Upright sleeping tops are nonlinearly orbitally stable if $\mathrm{I}_{3}$ is the maximal eigenvalue of $\mathbb{I}_{\mathrm{ref}}$ and

$$
\xi^{2}>\frac{m g \ell}{\mathrm{I}_{3}-\mathrm{I}_{\mathrm{m}}}
$$

where $I_{m}:=\max \left\{I_{1}, I_{2}\right\}$.

(ii) $\theta=\pi$ and $\phi=0$. Hanging sleeping tops are stable if either $\mathrm{I}_{3}$ is the maximal axis of inertia, or

$$
\xi^{2}<\frac{m g \ell}{\mathrm{I}_{\mathrm{m}}-\mathrm{I}_{3}}
$$

(iii) $\theta \neq 0, \pi$ and $\phi=0$. Tilted tops for which the centre of mass lies on the principal axis of inertia with eigenvalue $\mathrm{I}_{3}$ are stable if either $\mathrm{I}_{2}$ is the maximal axis of inertia, or

$$
\mathrm{I}_{3}>\mathrm{I}_{2}>\mathrm{I}_{1} \quad \text { and } \quad 1>\cos ^{2} \theta>\frac{\mathrm{I}_{2}}{3\left(\mathrm{I}_{3}-\mathrm{I}_{2}\right)}
$$

(iv) $\phi \neq 0$. Tilted tops for which the position vector of the centre of mass is orthogonal to the principal axis $\mathbf{e}_{1}$ are stable if

$$
\mathrm{I}_{3}>\mathrm{I}_{2} \quad \text { and } \quad \min \left\{\tau_{1}, \tau_{2}\right\}>\rho(\theta, \phi)
$$

or if

$$
\mathrm{I}_{2}>\mathrm{I}_{3} \quad \text { and } \quad \rho(\theta, \phi)>\max \left\{\tau_{1}, \tau_{2}\right\}
$$

where

$$
\rho(\theta, \phi):=\frac{\sin (\theta+\phi)}{\sin (\theta-\phi)} \quad \tau_{1}:=\frac{\mathrm{I}_{2}+\mathrm{I}_{3}-2 \mathrm{I}_{1}}{\mathrm{I}_{3}-\mathrm{I}_{2}}
$$

and

$$
\tau_{2}:=\frac{4\left(I_{3}-I_{2}\right) \sin ^{2} 2 \theta}{I_{2}+I_{3}+\cos 2 \theta}+\cos 2 \theta .
$$




\subsection{Stability of general relative equilibria}

An arbitrary relative equilibrium can be viewed as a point on a curve of relative equilibria parametrized by angular velocity. We shall characterize below all possible sequences of stability transitions along such curves. We make use of equations (2.27) and (2.26), which can be expressed in the form

$$
\begin{aligned}
\operatorname{tr} \widetilde{\mathscr{B}}_{e} & =\frac{4(g m \ell \sin \theta)^{2}}{\xi^{2} \mathbb{I}\left(\Lambda_{e}\right)}-\xi^{2}\left(\operatorname{tr} \mathbb{I}_{\Lambda_{e}}-3 \mathbb{I}\left(\Lambda_{e}\right)\right)-2 g m \ell \cos \theta \\
& =-\frac{(g m \ell)^{2}}{\kappa}\left(\kappa^{2} \sigma_{1}+2 \kappa \cos \theta-4 \sin ^{2} \theta\right)
\end{aligned}
$$

and

$$
\begin{aligned}
\operatorname{det} \widetilde{\mathscr{B}}_{e}=\frac{\xi^{4}}{\mathbb{I}\left(\Lambda_{e}\right)}\left(4 \operatorname{det} \mathbb{I}_{\Lambda_{e}}-3 \mathbb{I}\left(\Lambda_{e}\right) \operatorname{si} \mathbb{I}_{\Lambda_{e}}+2 \mathbb{I}\left(\Lambda_{e}\right)^{2} \operatorname{tr} \mathbb{I}_{\Lambda_{e}}-\mathbb{I}\left(\Lambda_{e}\right)^{3}\right) \\
\quad+\xi^{2} g m \ell\left(\operatorname{tr} \Pi_{\Lambda_{e}}-3 \Pi\left(\Lambda_{e}\right)\right) \cos \theta+(g m \ell \sin \theta)^{2}\left(1-4 \frac{g m \ell \cos \theta}{\xi^{2} \Pi\left(\Lambda_{e}\right)}\right) \\
=\frac{(g m \ell)^{2}}{\kappa}\left(\kappa^{3}\left(2 \sigma_{1}-3 \sigma_{2}+4 \sigma_{3}\right)+\kappa^{2} \sigma_{1} \cos \theta+\kappa-4 \sin ^{2} \theta \cos \theta\right)
\end{aligned}
$$

where $\operatorname{si} A:=\frac{1}{2}\left((\operatorname{tr} A)^{2}-\operatorname{tr} A^{2}\right)$ for all three by three matrices $M$ and

(i) the $\sigma_{i}$ 's are the scaled invariants of the inertia tensor

$$
\begin{aligned}
& \sigma_{1}:=\frac{\operatorname{tr} I_{\text {ref }}}{\mathbb{I}\left(\Lambda_{e}\right)}-3=\frac{I_{1}+I_{2}+I_{3}}{I I\left(\Lambda_{e}\right)}-3 \\
& \sigma_{2}:=\frac{\operatorname{si~} I_{\mathrm{ref}}}{I\left(\Lambda_{e}\right)^{3}}-3=\frac{I_{1} I_{2}+I_{1} I_{3}+I_{2} I_{3}}{I\left(\Lambda_{e}\right)^{2}}-3 \\
& \sigma_{3}:=\frac{\operatorname{det} I_{\text {ref }}}{I\left(\Lambda_{e}\right)^{3}}-1=\frac{I_{1} I_{2} I_{3}}{I\left(\Lambda_{e}\right)^{3}}-1
\end{aligned}
$$

where the $\mathrm{I}_{i}$ 's are the eigenvalues of $\mathbb{I}_{\text {ref }}$ (and hence of $\mathbb{I}_{\Lambda}$ for all $\Lambda$ )

(ii) $\kappa:=\frac{\xi^{2} \mathbb{I}\left(\Lambda_{e}\right)}{m g \ell}=\frac{\xi^{2} \gamma_{e} \cdot \mathbb{I}_{\mathrm{ref}} \gamma_{e}}{m g \ell}$ is the scaled kinetic energy

(iii) $\theta$ is the angle between $\mathbf{m}_{e}$ and $\mathbf{e}_{3}$, i.e. $\mathbf{m}_{e} \cdot \mathbf{e}_{3}=m \ell \cos \theta=\gamma_{e} \cdot \mathbf{M}$.

Expressions (2.55) and (2.54) are obtained by means of the identities

$$
\begin{aligned}
& \left.\operatorname{tr} S\right|_{\mathbf{v} \perp}=\operatorname{tr} S-(\mathbf{v} \cdot S \mathbf{v}) \\
& \left.\operatorname{det} S\right|_{\mathbf{v} \perp}=\operatorname{si} S-(\mathbf{v} \cdot S \mathbf{v}) \operatorname{tr} S+|S \mathbf{v}|^{2}
\end{aligned}
$$

and

$(\mathbf{v} \times S \mathbf{v}) \cdot S(\mathbf{v} \times S \mathbf{v})=\left.(\mathbf{v} \cdot S \mathbf{v}) \operatorname{det} S\right|_{\mathbf{v}}-\operatorname{det} S$

$$
=(\mathbf{v} \cdot S \mathbf{v})\left(\operatorname{si} S-(\mathbf{v} \cdot S \mathbf{v}) \operatorname{tr} S+|S \mathbf{v}|^{2}\right)-\operatorname{det} S
$$


for any symmetric matrix $S$ and unit vector v. Applying (2.58) and (2.57) to $\left.\mathbb{I}_{\Lambda_{e}}\right|_{\mathbf{e}_{1}-\mathbf{e}_{2}}$ and making use of the equilibrium identity $(2.30)$, we see that

$$
\begin{aligned}
\operatorname{det} \mathscr{C}_{e}=\xi^{4} \operatorname{det} & \left(\left.\mathbb{I}_{\Lambda_{e}}\right|_{\mathbf{e}_{1}-\mathbf{e}_{2}}\right)+\xi^{2} \Gamma_{e} \operatorname{tr}\left(\left.\mathbb{I}_{\Lambda_{e}}\right|_{\mathbf{e}_{1}-\mathrm{e}_{2}}\right)+\Gamma_{e}^{2} \\
= & \xi^{4}\left(\operatorname{si~} \mathbb{I}_{\Lambda_{e}}-\mathbb{I}\left(\Lambda_{e}\right) \operatorname{tr} \mathbb{I}_{\Lambda_{e}}+\mathbb{I}\left(\Lambda_{e}\right)^{2}\right)+(g m \ell \sin \theta)^{2} \\
& +\xi^{2} \Gamma_{e}\left(\operatorname{tr} \mathbb{I}_{\Lambda_{e}}-\mathbb{I}\left(\Lambda_{e}\right)\right)+\Gamma_{e}^{2} \\
= & \xi^{4}\left(\operatorname{si} \mathbb{I}_{\Lambda_{e}}-2 \mathbb{I}\left(\Lambda_{e}\right) \operatorname{tr} \mathbb{I}_{\Lambda_{e}}+3 \mathbb{I}\left(\Lambda_{e}\right)^{2}\right) \\
& +\xi^{2} g \mathbf{e}_{3} \cdot \mathbf{m}_{e}\left(\operatorname{tr} \mathbb{I}_{\Lambda_{e}}+\mathbb{I}\left(\Lambda_{e}\right)\right)+(g m \ell)^{2} .
\end{aligned}
$$

The identity (2.59) implies that

$$
\begin{aligned}
\left(\mathbf{e}_{3} \times \mathbb{I}_{\Lambda_{e}} \mathbf{e}_{3}\right) \cdot \mathscr{C}_{e}\left(\mathbf{e}_{3} \times \mathbb{I}_{\Lambda_{e}} \mathbf{e}_{3}\right) & \\
= & \xi^{2}\left(\mathbb{I}\left(\Lambda_{e}\right) \operatorname{si} \mathbb{I}_{\Lambda_{e}}-\mathbb{I}\left(\Lambda_{e}\right)^{2} \operatorname{tr} \mathbb{I}_{\Lambda_{e}}+\mathbb{I}\left(\Lambda_{e}\right)\left|\mathbb{I}_{\Lambda_{e}} \mathbf{e}_{3}\right|^{2}-\operatorname{det} \mathbb{I}_{\Lambda_{e}}\right) \\
& +\left(g \mathbf{m}_{e} \cdot \mathbf{e}_{3}-\xi^{2} \mathbb{I}\left(\Lambda_{e}\right)\right)\left|\mathbb{I}_{\Lambda_{e}} \mathbf{e}_{3}\right|^{2} \\
= & \xi^{2}\left(\mathbb{I}\left(\Lambda_{e}\right) \operatorname{si} \mathbb{I}_{\Lambda_{e}}-\mathbb{I}\left(\Lambda_{e}\right)^{2} \operatorname{tr} \mathbb{I}_{\Lambda_{e}}-\operatorname{det} \mathbb{I}_{\Lambda_{e}}+\mathbb{I}\left(\Lambda_{e}\right)^{3}\right) \\
& +\frac{(g m \ell)^{3} \cos \theta \sin ^{2} \theta}{\xi^{4}} .
\end{aligned}
$$

Equation (2.55) follows directly from (2.60) and (2.62).

We now use the expressions (2.54) and (2.55) to obtain a rough general classification of the possible behaviours of the system as the scaled kinetic energy $\kappa$ is increased. The classifications are determined by applying the Routh-Hurwitz criteria to the polynomials

$$
P_{\mathrm{t}}(\kappa):=-\sigma_{1} \kappa^{2}-2 \cos \theta \kappa+4 \sin ^{2} \theta
$$

and

$$
P_{\mathrm{d}}(\kappa):=\beta \kappa^{3}+\sigma_{1} \cos \theta \kappa^{2}+\kappa-4 \sin ^{2} \theta \cos \theta
$$

where $\beta:=2 \sigma_{1}-3 \sigma_{2}+4 \sigma_{3}$.

The Routh-Hurwitz criterion states that the number of roots of $P_{\mathrm{t}}$ and $P_{\mathrm{d}}$ in the right half-plane are given by the number of sign changes in the sequences

$\sigma_{1}, \cos \theta,-1 \quad$ and $\quad \beta, \sigma_{1} \cos \theta, \frac{\sigma_{1}+4 \beta \sin ^{2} \theta}{\sigma_{1}},-\cos \theta$

respectively. (For a discussion of the Routh-Hurwitz criterion, see, for example, Gantmacher (1989).)

For $\beta \cos \theta>0$, the Routh-Hurwitz criterion implies that $P_{\mathrm{d}}$ has either one or three positive real roots. For $\beta \cos \theta<0, P_{\mathrm{d}}$ has either zero or two positive real roots. $P_{\mathrm{d}}$ is positive (negative) for sufficiently large $\kappa$ if $\beta$ is positive (negative). For sufficiently small $\kappa, P_{\mathrm{d}}$ is positive (negative) for $\cos \theta$ negative (positive). We next note that the condition that the determinant have a local extremum, namely, that

$$
3 \beta \kappa^{2}+2 \sigma_{1} \kappa \cos \theta+1=0
$$


Table 1. Possible stability transitions for relative equilibria of general tops.

\begin{tabular}{lll}
\hline & $\cos \theta>0$ & $\cos \theta<0$ \\
\hline$\beta<0$ & I, IPI or INI & PI \\
$\beta>0$ and $\sigma_{1}<0$ & IP, IPIP or INIP & P or PIP \\
$\beta>0$ and $\sigma_{1}>0$ & IN & PIN \\
\hline
\end{tabular}

cannot be satisfied by tops for which $\beta$ and $\sigma_{1} \cos \theta$ are both positive, or

$$
\beta>\frac{1}{3} \sigma_{1}^{2} \cos ^{2} \theta .
$$

For such tops, the determinant can change sign at most once.

Ignoring the special cases associated to simultaneous roots of $P_{\mathrm{t}}$ and $P_{\mathrm{d}}$, or their derivatives, we summarize the possible stability transitions in table 1 . The entries of the table are interpreted as follows: $\mathrm{P}$ (respectively N) indicates positive (respectively negative) definiteness of $\widetilde{\mathscr{B}}_{e}$, while I indicates indefiniteness of $\widetilde{\mathscr{B}}_{e}$. The transition sequences read from left to right with increasing $\kappa$.

(i) Negative definiteness of the second variation is possible only if either

$$
\sigma_{1}>0 \quad \text { or } \quad \cos ^{2} \theta>\frac{4 \sigma_{1}}{4 \sigma_{1}-1}>0 .
$$

(ii) Multiple changes of signature are possible only if

$$
\cos ^{2} \theta>\frac{3 \beta}{\sigma_{1}^{2}} \text { and either } \sigma_{1}>0 \text { or } \cos ^{2} \theta>\frac{4 \beta+\sigma_{1}}{4 \beta} \text {. }
$$

For example, for $\beta>\sigma_{1}^{2} / 3, \sigma_{1}<0$, and $\cos \theta>0$, the only possible sequence is IP. The second inequality in equation (2.68) is obtained by requiring that the roots of $P_{\mathrm{t}}$ be real. Condition (2.69) implies that the second sequence in (2.65) has three sign changes and the polynomial (2.66) has real roots, i.e., that the polynomial $P_{\mathrm{d}}$ has two positive critical points.

Some fairly detailed information regarding the linear stability of the tops can be obtained from table 1, making use of the following observations. Positive definiteness of $\widetilde{\mathscr{B}}_{e}$ implies nonlinear, and hence linear, stability of the relative equilibrium. The characteristic polynomial (4.18) for the linearized dynamics, which is derived in section 4 , has both real and imaginary roots if det $\widetilde{\mathscr{B}}_{e}$ is negative; hence indefiniteness of $\widetilde{\mathscr{B}}_{e}$ implies linear instability. Negative definiteness of $\widetilde{\mathscr{B}}_{e}$ can be associated with either stability or instability. Some general conclusions regarding linear stability can be summarized as follows:

(i) $\cos \theta>0$ implies that the top is unstable at sufficiently low velocities

(ii) $\cos \theta<0$ implies that the top is stable at sufficiently low velocities

(iii) $\beta<0$ implies that the top is unstable at sufficiently high velocities

(iv) $\beta>0$ and $\sigma_{1}<0$ implies that the top is stable at sufficiently high velocities.

Remark: Many of the cases determined by the Routh-Hurwitz criterion can be ruled out as possible sequences of behaviour by taking into account the fact that the trace cannot change sign in a region where the determinant is positive; hence the transitions 
PN and NP cannot occur unless the trace and determinant are simultaneously equal to zero. We let the letter $\mathrm{Z}$ denote the state $\widetilde{\mathscr{B}}_{e}=\mathbf{0}$. A further condition on the existence of the transitions PN and NP is that the relevant root of $P_{d}$ also be a critical point of $P_{\mathrm{d}}$. Degenerate cases, in which, for example, a critical point of $P_{\mathrm{d}}$ is simultaneously a root of $P_{\mathrm{d}}$, may result in the following sequences: IZI, for $\beta<0$ and $\cos \theta>0$; IZIP, IPZP, or INZP, for $\beta>0, \sigma_{1}<0$, and $\cos \theta>0$; and $\mathrm{PZN}$, for $\beta<0, \sigma_{1}>0$, and $\cos \theta<0$.

\section{Lagrange tops}

We now consider a Lagrange top, i.e. a top satisfying

$$
R \mathrm{II}_{\mathrm{ref}} R^{T}=\mathbb{I}_{\mathrm{ref}}
$$

for all rotations $R$ about the axis $\mathbf{M}$. Set $R=\exp (\epsilon \widehat{\mathbf{M}})$, where exp denotes the exponential map exp : so(3) $\rightarrow \mathrm{SO}(3)$ given explicitly by Rodrigues' formula

$$
\exp \widehat{\omega}=1_{3}+\frac{\sin (|\omega|)}{|\omega|} \widehat{\omega}+2 \frac{\sin ^{2}(|\omega| / 2)}{|\omega|^{2}} \widehat{\omega}^{2}
$$

Differentiating with respect to $\epsilon$, we see that (3.1) holds if and only if

$$
\operatorname{sym}\left[\mathbb{I}_{\text {ref }} \widehat{\mathbf{M}}\right]=\mathbf{0} .
$$

The symmetry condition (3.1) also implies that $\mathbf{M}$ is an eigenvector of $\boldsymbol{I}_{\mathrm{ref}}$. Indeed, since $\mathbf{M}$ is the axis of rotation of $R$, we have $R \mathbf{M}=\mathbf{M}$; thus (3.1) implies that $R \mathbb{I}_{\text {ref }} \mathbf{M}=\mathbb{I}_{\text {ref }} \mathbf{M}$, i.e. $\mathbb{I}_{\text {ref }} \mathbf{M}$ is also the axis of the rotation $R$. Hence $\mathbb{I}_{\text {ref }} \mathbf{M}=\lambda_{M} \mathbf{M}$ for some $\lambda_{M} \in \mathbb{R}$. Moreover, the remaining two eigenvalues of $\mathbb{I}_{\mathrm{ref}}$ are necessarily equal, which can be shown as follows. If $\mathbb{I}_{\text {ref }}$ has three equal eigenvalues, then there is nothing to prove. If not, let $\lambda \neq \lambda_{M}$ be an eigenvalue of $\mathbb{I}_{\text {ref }}$ with eigenvector $\mathbf{x}$. The vector $\mathbf{x}$ is not parallel to $\mathbf{M}$; hence $\mathbf{x}$ and $R \mathbf{x}$ are linearly independent. The symmetry condition (3.1) implies that $\lambda R \mathbf{x}=R \Pi_{\mathrm{ref}} \mathbf{x}=\mathbb{I}_{\mathrm{ref}} R \mathbf{x}$; hence $R \mathbf{x}$ is a distinct eigenvector with the same eigenvalue $\lambda$. The reference symmetry condition (3.3) leads to the spatial symmetry condition $\operatorname{sym}\left[\mathbb{I}_{\Lambda} \widehat{\mathbf{m}}\right]=\mathbf{0}$. An analogous argument to that used for $\mathbf{M}$ and $\mathbb{I}_{\text {ref }}$ shows that $\mathbf{m}$ is an eigenvector of the current spatial inertia tensor $\mathbb{I}_{\Lambda}$.

The Hamiltonian (1.27) associated to such a top is invariant not only under left, i.e. spatial, rotations about the axis $\mathbf{e}_{3}$, but also under the right $S^{1}$ action of body rotation through the angle $\theta$ about the axis $\mathbf{M}$, given by

$$
\theta \cdot \Lambda:=\Lambda(\exp (\theta \widehat{\Sigma}))^{T}
$$

where $\Sigma:=\frac{1}{|\mathbf{M}|} \mathbf{M}$. Note that the induced action on the spatial representation $(\Lambda, \pi)$ is $\theta \cdot(\Lambda, \pi)=(\theta \cdot \Lambda, \pi)$ and the invariance condition for the Hamiltonian reads $H(\theta \cdot \Lambda, \pi)=H(\Lambda, \pi)$ for any $\theta \in S^{1}$. In this case, the full symmetry group of the Hamiltonian is $G=S^{1} \times S^{1}$, with Lie algebra $g=\mathbb{R}^{2}$ and infinitesimal generator

$$
(\xi, \omega)_{Q}(\Lambda)=\xi \hat{\mathbf{e}}_{3} \Lambda-\omega \Lambda \widehat{\Sigma}
$$


The locked inertia tensor $\mathbb{I}(\Lambda): \mathbb{R}^{2} \rightarrow \mathbb{R}^{2}$ is defined by the relationship

$$
\begin{aligned}
(\eta, \zeta) \cdot \mathbb{I}(\Lambda)(\xi, \omega) & =\left\langle\boldsymbol{F} L\left((\xi, \omega)_{Q}(\Lambda)\right),(\eta, \zeta)_{Q}(\Lambda)\right\rangle \\
& =\left\langle\Lambda\left(\xi \widehat{\Lambda^{T} \mathbf{e}_{3}}-\omega \widehat{\Sigma}\right), \Lambda\left(\eta \widehat{\Lambda}^{T} \mathbf{e}_{3}-\zeta \widehat{\Sigma}\right)\right\rangle_{\mathbf{I}} \\
& =\left(\xi \Lambda^{T} \mathbf{e}_{3}-\omega \Sigma\right) \cdot \mathbb{I}_{\mathrm{ref}}\left(\eta \Lambda^{T} \mathbf{e}_{3}-\zeta \Sigma\right)
\end{aligned}
$$

using the left $\operatorname{SO}(3)$ invariance of the inner product $\langle,\rangle_{\mathrm{I}}$, the formula $\Lambda \widehat{\mathbf{v}} \Lambda^{T}=\widehat{\Lambda \mathbf{v}}$, (1.14), and (1.19). Hence

$$
\mathbb{I}(\Lambda)=\left(\begin{array}{cc}
\mathbf{e}_{3} \cdot \Lambda \mathbb{I}_{\mathrm{ref}} \Lambda^{T} \mathbf{e}_{3} & -\mathbf{e}_{3} \cdot \Lambda \mathbb{I}_{\mathrm{ref}} \Sigma \\
-\mathbf{e}_{3} \cdot \Lambda \mathbb{I}_{\mathrm{ref}} \Sigma & \Sigma \cdot \mathbb{I}_{\mathrm{rer}} \Sigma
\end{array}\right)=\left(\begin{array}{cc}
\mathbf{e}_{3} \cdot \mathbb{I}_{\Lambda} \mathbf{e}_{3} & -\mathbf{e}_{3} \cdot \mathbb{I}_{\Lambda} \mathbf{s} \\
-\mathbf{e}_{3} \cdot \mathbb{I}_{\Lambda} \mathbf{s} & \mathbf{s} \cdot \mathbb{I}_{\Lambda} \mathbf{s}
\end{array}\right)
$$

where $s:=\Lambda \Sigma$.

We are now ready to determine the conditions of relative equilibrium for the Lagrange top. The augmented potential takes the form

$$
V_{(\xi, \omega)}(\Lambda)=\Lambda \mathbf{M} \cdot \mathbf{g}-\frac{1}{2}(\xi, \omega) \cdot \mathbb{I}(\Lambda)(\xi, \omega)
$$

with first variation

$$
D V_{(\xi, \omega)}\left(\Lambda_{e}\right) \cdot \widehat{\delta \theta} \Lambda_{e}=\left(\mathbf{e}_{3} \times \delta \theta\right) \cdot\left(g \mathbf{m}_{e}-\xi \mathbf{I}_{\Lambda_{e}}\left(\xi \mathbf{e}_{3}-\omega \mathbf{s}_{e}\right)\right)
$$

where $\mathrm{s}_{e}:=\Lambda_{e} \Sigma$. Hence the configuration $\Lambda_{e}$ is a relative equilibrium if and only if

$$
g \mathbf{m}_{e}-\xi \mathbf{I}_{\Lambda_{e}}\left(\xi \mathbf{e}_{3}-\omega \mathbf{s}_{e}\right)=\Gamma_{e} \mathbf{e}_{3}
$$

where

$$
\begin{aligned}
\Gamma_{e}: & =\left(g \mathbf{m}_{e}-\xi \mathbb{I}_{\Lambda_{e}}\left(\xi \mathbf{e}_{3}-\omega \mathbf{s}_{e}\right)\right) \cdot \mathbf{e}_{3} \\
& =m g \ell \mathbf{s}_{e} \cdot \mathbf{e}_{3}-(\xi, 0) \cdot \mathbb{I}\left(\Lambda_{e}\right)(\xi, \omega)
\end{aligned}
$$

While the equilibrium conditions (3.10) can be expressed as two scalar equations (namely, that the $e_{1}$ and $e_{2}$ components of the left hand side equal zero), we shall see that, due to the symmetry of the Lagrange top, relative equilibria are determined by a single non-trivial scalar equation.

We now discuss two distinct classes of relative equilibria for Lagrange tops: precessing tops and sleeping tops. The motion of the spatial axis of symmetry of a precessing top traces out a cone about the axis of gravity; the spatial axis of symmetry $s_{e}$ of a sleeping Lagrange top coincides with the axis of gravity $\mathbf{e}_{3}$. It is convenient to distinguish precessing and sleeping tops when considering the equilibrium conditions; it is essential to distinguish them when performing the stability analysis. The crucial distinction is the additional symmetry of the sleeping top: spatial rotations about the axis of gravity are indistinguishable from body rotations about the axis of symmetry. For example, a spatial rotation combined with a reverse body rotation through the same angle fixes the sleeping top. A stability analysis that neglects this additional symmetry may yield overly restrictive stability conditions. We first treat the case of a precessing top, using the standard stability algorithm, and then consider the sleeping Lagrange top. 


\subsection{The precessing Lagrange top}

If the axis of gravity and the axis of spatial symmetry are distinct, i.e., $\mathbf{s}_{e} \neq \pm \mathbf{e}_{3}$, then the group $G$ acts freely in a neighbourhood of $\Lambda_{e}$. The two-dimensional tangent space $g \cdot \Lambda_{e}$ to the group orbit is given by (3.5);

$$
g \cdot \Lambda_{e}=\operatorname{span}\left\{\widehat{\mathbf{e}}_{3} \Lambda_{e}, \widehat{\mathbf{s}}_{e} \Lambda_{e}\right\}
$$

Since the group $G$ is abelian, the augmented and amended potentials are $G$ invariant and $g \cdot \Lambda_{e}$ lies in the kernel of the first and second variations of the modified potentials. Thus the spatial representation of the space of non-trivial variations is spanned by $\delta \Lambda_{v}:=\widehat{\delta \theta}_{v} \Lambda_{e}$, where $\delta \theta_{v}:=\left(\mathbf{e}_{3} \times \mathbf{s}_{e}\right) /\left|\mathbf{e}_{3} \times \mathbf{s}_{e}\right|$, and $\Lambda_{e}$ is a relative equilibrium if and only if the single scalar equation

$$
D V_{(\xi, \omega)}\left(\Lambda_{e}\right) \cdot \delta \Lambda_{v}=0
$$

is satisfied.

We now show that any steadily precessing Lagrange top is orbitally nonlinearly stable. As we did for the tilted asymmetric relative equilibria, we choose a coordinate system that simplifies the necessary calculations. Set

$$
\mathbf{e}_{1}:=\delta \theta_{v}=\frac{\mathbf{e}_{3} \times \mathbf{s}_{e}}{\left|\mathbf{e}_{3} \times \mathbf{s}_{e}\right|} \quad \text { and } \quad \mathbf{e}_{2}:=\mathbf{e}_{3} \times \mathbf{e}_{1} .
$$

Thus $\mathbf{s}_{e}=R_{\theta} \mathbf{e}_{3}$ for some $\theta \neq 0, \pi$, where $R_{\theta}$ is given by (2.37). The symmetry assumptions imply that the reference inertia matrix $\mathbb{I}_{\text {ref }}$ has a double eigenvalue, say $I_{1}$, and a distinct eigenvalue, say $I_{3}$, and that $\operatorname{sym}\left[\mathbb{I}_{\Lambda_{e}} \hat{\mathbf{s}}_{e}\right]=\mathbf{0}$. Hence we set $\mathrm{I}_{\mathrm{ref}}=\operatorname{diag}\left[\mathrm{I}_{1}, \mathrm{I}_{1}, \mathrm{I}_{3}\right]$ and $\Lambda_{e}=R_{\theta}$.

Equation (3.7) implies that the equilibrium locked inertia tensor $\mathbb{I}\left(\Lambda_{e}\right)$ is given by

$$
\mathrm{I}\left(\Lambda_{e}\right)=\left(\begin{array}{cc}
\mathrm{I}_{1} \sin ^{2} \theta+\mathrm{I}_{3} \cos ^{2} \theta & -\mathrm{I}_{3} \cos \theta \\
-\mathrm{I}_{3} \cos \theta & \mathrm{I}_{3}
\end{array}\right) .
$$

The non-trivial equilibrium condition is given by

$D V_{(\xi, \omega)}\left(\Lambda_{e}\right) \cdot \delta \Lambda_{v}=\sin \theta\left(\xi^{2}\left(\mathrm{I}_{3}-\mathrm{I}_{1}\right) \cos \theta-\xi \omega \mathrm{I}_{3}-m g \ell\right)=0$

which is obtained by substituting $\mathbf{m}_{e}=m \ell \mathbf{s}_{e}=m \ell R_{\theta} \mathbf{e}_{3}$ into (3.9). Neglecting for the moment the solutions $\theta=0, \pi$ corresponding to the sleeping Lagrange top, the equilibrium condition (3.16) may be solved for $\omega$ to yield

$$
\omega=\frac{\xi^{2}\left(\mathrm{I}_{3}-\mathrm{I}_{1}\right) \cos \theta-m g \ell}{\xi \mathrm{I}_{3}} .
$$

Hence, $\boldsymbol{F} L\left((\xi, \omega)_{Q}\left(\Lambda_{e}\right)\right)$ is a precessing relative equilibrium if and only if $\omega$ satisfies (3.17). The equilibrium condition (3.16) and the following stability result can be found in Routh (1884, section 207), with the change of variables $\mu=\xi$ and $n=\xi \cos \theta-\omega$.

The relative equilibrium with configuration $\Lambda_{e}$ is orbitally stable if

$$
\begin{aligned}
\mathscr{B}_{e}\left(\delta \Lambda_{v}, \delta \Lambda_{v}\right)= & D^{2} V_{(\xi, \omega)}\left(\Lambda_{e}\right)\left(\delta \Lambda_{v}, \delta \Lambda_{v}\right) \\
& +\left(D \mathbb{I}_{(\xi, \omega)}\left(\Lambda_{e}\right) \cdot \delta \Lambda_{v}\right) \cdot \mathbb{I}\left(\Lambda_{e}\right)^{-1}\left(D \mathbb{I}_{(\xi, \omega)}\left(\Lambda_{e}\right) \cdot \delta \Lambda_{v}\right)>0
\end{aligned}
$$


where $\mathbb{I}_{(\xi, \omega)}(\Lambda):=\mathbb{I}(\Lambda)(\xi, \omega)$ is the locked momentum map for the $G$ action. The second variation of the augmented potential (3.8) takes the form

$D^{2} V_{\langle\xi, \omega)}\left(\Lambda_{e}\right)\left(\widehat{\delta \theta} \Lambda_{e}, \widehat{\Delta \theta} \Lambda_{e}\right)=-\left(\mathbf{e}_{3} \times \delta \theta\right) \cdot\left(\xi^{2} \mathbb{I}_{\Lambda_{e}}+\Gamma_{e} \mathbf{1}_{3}\right)\left(\mathbf{e}_{3} \times \Delta \theta\right)$

where $\Gamma_{e}=-\xi^{2} I_{1}$ is determined using (3.11) and (3.17). Hence (3.19) implies that the second variation of the augmented potential in the direction of the non-trivial mode $\delta \Lambda_{v}=\widehat{\mathbf{s}}_{1} \Lambda_{e}$ is given by

$\left.D^{2} V_{(\xi, \omega)}\left(\Lambda_{e}\right)\left(\delta \Lambda_{v}, \delta \Lambda_{v}\right)\right)=-\mathbf{e}_{2} \cdot\left(\xi^{2} \mathbb{I}_{\Lambda_{e}}+\Gamma_{e} \mathbf{1}_{3}\right) \mathbf{e}_{2}=\xi^{2}\left(\mathrm{I}_{1}-\mathrm{I}_{3}\right) \sin ^{2} \theta$

The locked momentum map $\mathbb{I}_{(\xi, \omega)}$ has first variation

$D \mathbb{I}_{(\tilde{\xi}, \omega)}\left(\Lambda_{e}\right) \cdot \widehat{\delta \theta} \Lambda_{e}=\left(\begin{array}{c}2 \xi\left(\mathbf{e}_{3} \times \delta \theta\right) \cdot \mathbb{I}_{\Lambda_{e}} \mathbf{e}_{3}-\omega\left(\mathbf{e}_{3} \times \delta \theta\right) \cdot \mathbb{I}_{\Lambda_{e}} \mathbf{s}_{e} \\ -\xi\left(\mathbf{e}_{3} \times \delta \theta\right) \cdot \mathbb{I}_{\Lambda_{e}} \mathbf{s}_{e}\end{array}\right)$

In particular, for $\omega$ given by (3.17),

$$
D \mathbb{I}_{(\xi, \omega)}\left(\Lambda_{e}\right) \cdot \delta \Lambda_{v}=\xi \sin \theta\left(\begin{array}{c}
\left(\mathrm{I}_{1}-\mathrm{I}_{3}\right) \cos \theta-\frac{m g \ell}{\xi^{2}} \\
\mathrm{I}_{3}
\end{array}\right)
$$

and hence

$$
\begin{aligned}
\left(D \mathbb{I}_{(\xi, \omega)}\left(\Lambda_{e}\right)\right. & \left.\cdot \delta \Lambda_{v}\right) \cdot \mathbb{I}\left(\Lambda_{e}\right)^{-1}\left(D \mathbb{I}_{(\xi, \omega)}\left(\Lambda_{e}\right) \cdot \delta \Lambda_{v}\right) \\
& =\xi^{2}\left(\mathrm{I}_{1} \cos ^{2} \theta+\mathrm{I}_{3} \sin ^{2} \theta\right)-2 m g \ell \cos \theta+\frac{(m g \ell)^{2}}{\xi^{2} \mathrm{I}_{1}} .
\end{aligned}
$$

Thus, using (3.18), we obtain

$$
\begin{aligned}
\mathscr{B}_{e}\left(\delta \Lambda_{v}, \delta \Lambda_{v}\right) & =\xi^{2} \mathrm{I}_{1}-2 m g \ell \cos \theta+\frac{(m g \ell)^{2}}{\xi^{2} \mathrm{I}_{1}} \\
= & \frac{1}{\xi^{2} \mathrm{I}_{1}}\left(\left(\xi^{2} \mathrm{I}_{1}-m g \ell \cos \theta\right)^{2}+(m g \ell \sin \theta)^{2}\right)>0
\end{aligned}
$$

for all $\theta \neq 0, \pi$. It follows that all tilted Lagrange tops in steady precession are nonlinearly stable modulo rotations about the axis of gravity and the axis of symmetry.

\subsection{The sleeping Lagrange top}

We consider a steady motion in which the axis of symmetry, the centre of mass vector, and the axis of gravity all coincide. The spatial symmetry condition $\operatorname{sym}\left[\mathbb{I}_{\Lambda_{e}} \widehat{\mathbf{s}}_{e}\right]=\mathbf{0}$ implies that $\mathbf{e}_{3}$ is an eigenvector of $\mathbb{I}_{\Lambda_{e}}$. Hence we can assume without loss of generality that $\mathbb{I}_{\Lambda_{e}}$ is diagonal; the symmetry assumption then implies that $\mathbb{I}_{\Lambda_{e}}=\operatorname{diag}\left[\mathrm{I}_{1}, \mathrm{I}_{1}, \mathrm{I}_{3}\right]$ for some constants $I_{1}, I_{3} \in \mathbb{R}$.

As was previously discussed, the group $G=S^{1} \times S^{1}$ fails to act freely when the axis of gravity and the spatial axis of symmetry coincide. In this case, $s_{e}= \pm e_{3}$ and hence $\mathbf{m}_{e}= \pm m \ell \mathbf{e}_{3}$. We shall analyse in detail the case $\mathbf{s}_{e}=\mathbf{e}_{3}$ and simply present the results for the case $s_{e}=-\mathbf{e}_{3}$, which are derived analogously, at the end of the section. If $\mathbf{s}_{e}=\mathbf{e}_{3}$, then

$$
(\xi, \xi)_{Q}\left(\Lambda_{e}\right)=\xi\left(\widehat{\mathbf{e}}_{3}-\widehat{\mathbf{s}}_{e}\right) \Lambda_{e}=\mathbf{0} ;
$$


hence the tangent space $g \cdot \Lambda_{e}$ to the group orbit is only one-dimensional. This has the crucial consequence that the inertia tensor is no longer invertible;

$$
\begin{aligned}
\mathbb{I}\left(\Lambda_{e}\right) & =\left(\begin{array}{cc}
\mathbf{e}_{3} \cdot \mathbb{I}_{\Lambda_{e}} \mathbf{e}_{3} & -\mathbf{e}_{3} \cdot \mathbb{I}_{\Lambda_{e}} \mathbf{s}_{e} \\
-\mathbf{e}_{3} \cdot \Lambda_{e} \mathbb{I}_{\Lambda_{e}} \mathbf{s}_{e} & \mathbf{s}_{e} \cdot \mathbb{I}_{\Lambda_{e}} \mathbf{s}_{e}
\end{array}\right) \\
& =\left(\mathbf{e}_{3} \cdot \mathbb{I}_{\Lambda_{e}} \mathbf{e}_{3}\right)\left(\begin{array}{cc}
1 & -1 \\
-1 & 1
\end{array}\right)
\end{aligned}
$$

has a non-trivial kernel equal to $\operatorname{span}\{(1,1)\}$. Hence we no longer have a one to one correspondence between angular velocities and angular momenta. Given $\lambda \in \mathbb{R}$, define $\mathbf{v}_{\lambda}:=(\lambda, \lambda-1)$; it follows from (3.25) that the motion of the sleeping Lagrange top determined by the infinitesimal generator $\xi \mathbf{v}_{\lambda}$ is the same as that generated by a pure spatial rotation with angular velocity $\xi \mathbf{e}_{3}$, regardless of the choice of $\lambda$. Hence, when considering a sleeping Lagrange top rotating with spatial angular velocity $\xi$, we are free to choose an arbitrary value of $\lambda$ and take $\xi \mathbf{v}_{\lambda}$ as the generator of the relative equilibrium.

The reduced energy momentum method can still be applied to systems for which the group action fails to be free; however, some slight modifications to the method are required. The treatment of symmetric equilibria is described in Lewis (1991a); while the definitions there are given for Lagrangian systems, they are readily translated into the Hamiltonian context. For symmetric equilibria of a system with Hamiltonian of the form 'kinetic plus potential', relative equilibria still correspond to critical points of the augmented potential; the only change in the expression for the stability form $\mathscr{B}_{e}$ is in the momentum correction term. In the case of the sleeping Lagrange top, we shall show that this change is, in fact, transparent.

We consider the one-parameter family of augmented potentials $V_{\xi \mathbf{v}_{*}}$. Any sleeping top is a relative equilibrium, since (3.21) implies that $D \mathbb{I}_{(\xi, \omega)}\left(\Lambda_{e}\right) \equiv \mathbf{0}$ for any $(\xi, \omega) \in \mathbb{R}^{2}$ and $\Lambda_{e} \mathbf{e}_{3}=\mathbf{e}_{3}$. Thus, using (3.9), we obtain

$$
D V_{\xi \mathbf{v}_{\lambda}}\left(\Lambda_{e}\right)=m g \ell\left(\Lambda_{e} \mathbf{e}_{3}\right) \times \mathbf{e}_{3}-\xi^{2} \mathbf{v}_{\lambda} \cdot D \mathbb{I}\left(\Lambda_{e}\right) \mathbf{v}_{\lambda}=\mathbf{0}
$$

We now determine the stability conditions. Since the tangent to the group orbit $g \cdot \Lambda_{e}$ is one-dimensional if $\Lambda_{e}$ corresponds to a sleeping configuration of a Lagrange top, even though $g$ is two-dimensional, the space of non-trivial variations of $V_{\xi}$ is twodimensional for such equilibria. We now briefly discuss the definition of the stability form $\mathscr{B}_{e}$ at a symmetric equilibrium. Since $\mathbb{I}\left(\Lambda_{e}\right)$ is not invertible if the configuration is symmetric, the inverse $\mathbb{I}\left(\Lambda_{e}\right)^{-1}$ appearing in (2.7) must be replaced by a generalized inverse $\widetilde{\mathbb{I}\left(\Lambda_{e}\right)^{-1}}:$ range $\mathbb{I}\left(\Lambda_{e}\right) \rightarrow \mathrm{g} /\left(\operatorname{ker} \mathbb{I}\left(\Lambda_{e}\right)\right)$. However, since $D \mathbb{I}_{\left(\xi \mathrm{v}_{e}\right)}\left(\Lambda_{e}\right) \equiv \mathbf{0}$, the stability form $\mathscr{B}_{e}$ associated to a sleeping Lagrange top is equal to the second variation $D^{2} V_{\xi \mathbf{v}_{i}}\left(\Lambda_{e}\right)$. Using (3.19) and (3.11), we see that

$$
\Gamma_{e}=m g \ell-\xi^{2} \lambda \mathrm{I}_{3}
$$

and hence

$$
\begin{gathered}
D^{2} V_{\xi \mathbf{v},}\left(\Lambda_{e}\right)\left(\widehat{\delta \theta} \Lambda_{e}, \widehat{\Delta \theta} \Lambda_{e}\right)=-\left(\mathbf{e}_{3} \times \delta \theta\right) \cdot\left[\xi^{2} \lambda^{2} \mathbb{I}_{\Lambda_{e}}+\Gamma_{e} \mathbf{1}_{3}\right]\left(\mathbf{e}_{3} \times \Delta \theta\right) \\
=\left[\xi^{2} \lambda\left(\mathrm{I}_{3}-\lambda \mathbf{I}_{1}\right)-m g \ell\right]\left(\mathbf{e}_{3} \times \delta \theta\right) \cdot\left(\mathbf{e}_{3} \times \Delta \theta\right)
\end{gathered}
$$


Hence $D^{2} V_{\xi v_{\lambda}}\left(\Lambda_{e}\right)$ is positive semi-definite, with kernel equal to $\operatorname{span}\left\{\widehat{\mathbf{e}}_{3} \Lambda_{e}\right\}$, for $\lambda_{\bar{\xi}}<\lambda<\lambda_{\xi}^{+}$, where

$$
\lambda_{\xi}^{ \pm}:=\frac{\mathrm{I}_{3} \pm \sqrt{\mathrm{I}_{3}^{2}-4 m g \ell \mathrm{I}_{1} / \xi^{2}}}{2 \mathrm{I}_{1}} .
$$

The relative equilibrium is nonlinearly stable if we can find any real number $\lambda$ such that $D^{2} V_{\xi v_{\lambda}}\left(\Lambda_{e}\right)$ is positive definite; this is clearly possible if $\lambda \frac{F}{\xi}$ are real valued and distinct, i.e., if

$$
\xi^{2}>\frac{4 m g \ell \mathrm{I}_{1}}{\mathrm{I}_{3}^{2}}
$$

This is the well-known linear stability condition for an upright sleeping Lagrange top. Condition (3.31) can also be derived by the energy-Casimir method, as in Holm et al (1985), or by a linear stability analysis, as in section 4 .

Remark: Note that if one ignores the symmetry of the equilibrium and considers only the case $\lambda=1$ corresponding to a pure left rotation, then the apparent condition of stability, associated to positive definiteness of (3.29), is

$$
\xi^{2}>\frac{m g \ell}{\mathrm{I}_{3}-\mathrm{I}_{1}} .
$$

Clearly, failure to utilize all symmetries of the equilibrium can result in an overly restrictive stability condition. (As the top approaches a planar disc $\left(\mathrm{I}_{3}=2 \mathrm{I}_{1}\right)$, the $\xi$ value for loss of stability coincides with the $\xi$ value for a pure left rotation.)

The stability form for a hanging sleeping top is obtained by replacing $\ell$ with $-\ell$ in (3.30). Setting $\lambda=0$, we see that the hanging Lagrange top is always stable.

A Lagrange top with reference inertia tensor diag $\left[\mathrm{I}_{1}, \mathrm{I}_{1}, \mathrm{I}_{3}\right]$ tilted by an angle $\theta \neq 0, \pi$ about the $\mathbf{e}_{1}$ axis with spatial (left) angular velocity $\xi$ and spin (right) angular velocity $\omega$ remains in nonlinearly orbitally stable steady motion if

$$
\omega=\frac{\xi^{2}\left(\mathrm{I}_{3}-\mathrm{I}_{1}\right) \cos \theta-m g \ell}{\xi \mathrm{I}_{3}} .
$$

A sleeping top $(\theta=0, \pi)$ can be maintained in steady rotation at any angular velocity $\xi$. The upright sleeping top, with $\theta=0$, is orbitally stable if

$$
\xi^{2}>\frac{4 m g \ell \mathrm{I}_{1}}{\overline{\mathrm{I}}_{3}^{2}}
$$

The hanging sleeping top, with $\theta=\pi$, is always stable. 


\section{Linearized stability}

The energy analysis provides sufficient conditions for orbital nonlinear stability of relative equilibria. However, these conditions need not be necessary. If the energy analysis fails, i.e., if the stability form $\mathscr{B}_{e}$ fails to be positive definite on some complement to the tangent space $g \cdot \Lambda_{e}$ to the group orbit, then it is necessary to consider other criteria to determine the stability or instability of the relative equilibrium. In this section, we consider the linear stability of relative equilibria. As was shown in section 3.2 , in the case of a sleeping Lagrange top, the nonlinear stability conditions given by the energy analysis coincide with the classical linear stability criteria. However, indefiniteness of $\mathscr{B}_{e}$ is not always a reliable indicator of linear instability. The noncanonical structure of the reduced manifold can introduce 'magnetic' terms that may shift the spectrum.

We do not carry out the linear stability analysis on the canonical equations of motion (1.33) or (1.32); instead, we construct a reduced system of equations on a manifold $\mathscr{P}$ that is isomorphic to the quotient manifold $T^{*} Q / S^{1}$ and linearize this reduced set of equations. In the process of this reduction, we perform a momentum shift; the resulting linearized equations can be directly related to the stability matrix $\widetilde{\mathscr{B}}_{e}$ that appears in the energy stability analysis. The reduced variables are defined with respect to the body frame of reference within a moving frame with total momentum $\mu$. These variables are the unit direction of gravity $\gamma$ and the shifted momentum $\widetilde{\Pi}$, which are related to the canonical variables by the mapping

$$
\Sigma_{\mu}:(\Lambda, \Lambda \widehat{\Pi}) \mapsto(\gamma, \widetilde{\mathbf{\Pi}})=\left(\Lambda^{T} \mathbf{e}_{3}, \Pi-\mu \zeta\left(\Lambda^{T} \mathbf{e}_{3}\right)\right)
$$

where the vector field $\zeta: \mathbb{R}^{3} \rightarrow \mathbb{R}^{3}$ given by

$$
\zeta(\gamma):=\left(\gamma \cdot \mathbb{I}_{\mathrm{ref}} \gamma\right)^{-1} \mathbb{I}_{\mathrm{ref}} \gamma=I_{\gamma}^{-1} \mathbb{I}_{\mathrm{ref}} \gamma
$$

for $I_{\gamma}:=\gamma \cdot \mathbb{I}_{\text {ref }} \gamma$, satisfies

$$
\left(\Lambda, \mu \Lambda \widehat{\zeta}\left(\Lambda^{T} \mathbf{e}_{3}\right)\right)=\boldsymbol{F} L\left(\left(\mathbb{I}(\Lambda)^{-1} \mu\right)_{Q}(\Lambda)\right)
$$

(Note that $I_{\gamma}$ is the body expression of the inertia tensor $\mathbb{I}(\Lambda)$.) The motivation for this change of variables is discussed in section 6.2. The mapping (4.1) is invariant under spatial rotations about the axis of gravity; in fact,

$$
\Sigma_{\mu}\left(\Lambda_{1}, \Lambda_{1} \widehat{\Pi}_{1}\right)=\Sigma_{\mu}\left(\Lambda_{2}, \Lambda_{2} \widehat{\Pi}_{2}\right) \Longleftrightarrow\left(\Lambda_{2}, \Pi_{2}\right)=R_{\theta} \cdot\left(\Lambda_{1}, \Pi_{1}\right)=\left(R_{\theta} \Lambda_{1}, \Pi_{1}\right)
$$

for some rotation $R_{\theta}$ about $\mathbf{e}_{3}$. The set of all pairs $(\gamma, \widetilde{\Pi})$ makes up the Poisson reduced manifold

$$
\mathscr{P}:=\left\{(\gamma, \widetilde{\Pi}):|\gamma|=1, \tilde{\Pi} \in \mathbb{R}^{3}\right\} \approx S^{2} \times \mathbb{R}^{3} .
$$

It follows from (4.4) that the space $\mathscr{P}$ can be identified with the quotient manifold $T^{*} Q / S^{1}$.

The dynamics on $\mathscr{P}$ induced by the dynamics (1.32) on $T^{*} Q$ are given by

$\dot{\gamma}=\gamma \times \widetilde{\mathbf{\Omega}} \quad$ and $\quad \widetilde{\Pi}=\widetilde{\Pi} \times \widetilde{\mathbf{\Omega}}+\gamma \times\left(g \mathbf{M}-\xi^{2} \mathbb{I}_{\text {ref }} \gamma-\xi r_{\gamma} \widetilde{\Omega}\right)$ 
where

(i) $\widetilde{\Omega}:=\mathbb{I}_{\text {ref }}^{-1} \widetilde{\Pi}$ is the shifted angular velocity in the body frame

(ii) $\xi:=I_{\gamma}^{-1} \mu$ is the 'background velocity' associated to the momentum shift, i.e.

$$
\dot{\Lambda}=\Lambda(\widehat{\widetilde{\Omega}}+\xi \widehat{\gamma})
$$

(iii) $\tilde{r}_{\gamma}:=\mathrm{tir} \mathbb{H}_{\text {ref }}-2 I_{\gamma}^{-1}\left|\mathbb{I}_{\text {ref }} \hat{\gamma}\right|^{2}$.

These equations are geometrically derived and motivated in section 6.2 ; this derivation is not central to the remainder of the paper and may be skipped at the reader's discretion.

The linearized equations of motion associated to a relative equilibrium with angular velocity $\xi$ are obtained by linearizing (4.6) at the pair $\left(\gamma_{e}, \mathbf{0}\right)$ associated to a relative equilibrium with spatial coordinates $\left(\Lambda_{e}, \xi \mathbb{I}_{\Lambda_{e}} \mathbf{e}_{3}\right)$ and momentum $\mu=I_{\gamma_{e}} \xi$, yielding

$$
\left(\begin{array}{c}
\delta y \\
\dot{\delta \Pi}
\end{array}\right)=\left(\begin{array}{c}
\gamma_{e} \times \mathbb{I}_{\mathrm{ref}}^{-1} \widetilde{\delta \Pi} \\
\gamma_{e} \times\left(\mathrm{B}_{e} \delta \gamma-\xi r_{\gamma_{e}} \mathbb{I}_{\mathrm{ref}}^{-1} \widetilde{\delta \Pi}\right)
\end{array}\right) .
$$

Ignoring the null vector $\left(0, \mathbb{I}_{\mathrm{ref}} \gamma_{e}\right)$ associated to variations of the 'background velocity', we see that the linearized dynamics are determined by the four by four matrix

$$
L_{H}=\left(\begin{array}{cc}
\chi_{\gamma_{e}} & \mathbf{0} \\
\mathbf{0} & \chi_{\gamma_{e}}
\end{array}\right)\left(\begin{array}{cc}
\mathbf{0} & \mathbb{I}_{\perp}^{-1} \\
\mathrm{~B}_{e} & -\xi r_{\gamma_{e}} \mathbb{I}_{\perp}^{-1}
\end{array}\right)
$$

Here $\mathbb{I}_{\perp}^{-1}, \chi_{\gamma_{e}}$, and $\mathrm{B}_{e}$ denote the restrictions to the orthogonal complement to $\gamma_{e}$ of $\mathbb{I}_{\text {ref }}^{-1}, \widehat{\gamma}_{e}$, and the symmetric matrix

$\xi^{2}\left(4 I_{\gamma_{e}}^{-1}\left(\mathbb{I}_{\mathrm{ref}} \gamma_{e}\right) \otimes\left(\mathbb{I}_{\mathrm{ref}} \gamma_{e}\right)-\mathbb{I}_{\mathrm{ref}}+I_{\gamma_{e}} \mathbf{1}_{3}\right)-\left(g \mathbf{M} \cdot \gamma_{e}\right) \mathbf{1}_{3}=\xi^{2}\left(\mathbb{I}_{\mathrm{ref}}-2 I_{\gamma_{e}} \nabla \zeta\left(\gamma_{e}\right)\right)+\Gamma_{e} \mathbf{1}_{3}$

where the symmetric matrix

$$
\nabla \zeta(\gamma)=I_{\gamma}^{-1} \Pi_{\mathrm{ref}}-2 \zeta(\gamma) \otimes \zeta(\gamma)
$$

is the matrix representation of the first variation of the map $\zeta$ and $\Gamma_{e}$ is given by (2.13). The characteristic polynomial of the matrix $L_{H}$ is

$$
\chi(\lambda)=\frac{I_{\gamma_{e}}}{\operatorname{det} \mathbb{I}_{\mathrm{ref}}}\left(\lambda^{4} I_{\gamma_{e}}^{-1} \operatorname{det} \mathbb{I}_{\mathrm{ref}}+\lambda^{2}\left(\xi^{2} r_{\gamma_{e}}^{2}+\left\langle\mathrm{B}_{e}, \mathrm{~S}_{e}\right\rangle\right)+\operatorname{det} \mathrm{B}_{e}\right)
$$

where $\mathrm{S}_{e}$ is the restriction of the linear mapping

$$
\mathbb{I}_{\mathrm{ref}}-I_{\gamma_{e}}^{-1}\left(\mathbb{I}_{\mathrm{ref}} \gamma_{e}\right) \otimes\left(\mathbb{I}_{\mathrm{ref}} \gamma_{e}\right)
$$

to the orthogonal complement to $\gamma_{e}$. The polynomial $\chi$ has purely imaginary roots if and only if $\mathrm{B}_{e}$ has positive determinant and

$$
\xi^{2} r_{\gamma_{e}}^{2}+\left\langle\mathrm{B}_{e}, \mathrm{~S}_{e}\right\rangle>2 \sqrt{I_{\gamma_{e}}^{-1} \operatorname{det} \mathrm{I}_{\mathrm{ref}} \operatorname{det} \mathrm{B}_{e}}
$$


To relate the linear stability conditions as directly as possible to the energy stability conditions derived in section 2.2 , we can rewrite the characteristic polynomial (4.12) in terms of the spatial variables as follows. Given $\Lambda_{e} \in Q$, let $\mathscr{S}_{e}$ denote the restriction of the mapping

$$
\mathbf{I}_{\Lambda_{e}}-\mathbb{I}\left(\Lambda_{e}\right)^{-1}\left(\mathbb{I}_{\Lambda_{e}} \mathbf{e}_{3}\right) \otimes\left(\mathbf{I}_{\Lambda_{e}} \mathbf{e}_{3}\right)
$$

to the $\mathbf{e}_{1}-\mathbf{e}_{2}$ plane. Positive definiteness of $\mathbb{I}_{\Lambda_{e}}$ implies that

$$
\delta \pi \cdot \mathscr{S}_{e} \delta \pi=\mathbb{I}\left(\Lambda_{e}\right)^{-1} \operatorname{det} \mathbb{I}_{\Lambda_{e}}\left(\mathbf{e}_{3} \times \delta \pi\right) \cdot \mathbb{I}_{\Lambda_{e}}^{-1}\left(\mathbf{e}_{3} \times \delta \pi\right)>0
$$

for all $\delta \pi$ in the $\mathbf{e}_{1}-\mathbf{e}_{2}$ plane; hence $\mathscr{S}_{e}$ is positive definite. Define

$$
\rho_{e}:=\operatorname{tr} \mathbb{I}_{\Lambda_{e}}-2 \mathbb{I}\left(\Lambda_{e}\right)^{-1}\left|\mathbb{I}_{\Lambda_{e}} \mathbf{e}_{3}\right|^{2}
$$

If $\gamma_{e}=\Lambda_{e} \mathbf{e}_{3}$, then $\mathbb{I}\left(\Lambda_{e}\right)=I_{\gamma_{e}}, \rho_{e}=r_{\gamma_{e}}$, and $\mathscr{S}_{e}=\Lambda_{e} S_{e} \Lambda_{e}^{T}$. From (1.15) and (2.17) we obtain $\mathbb{I}_{\Lambda_{e}}=\Lambda_{e} \mathbb{I}_{\mathrm{ref}} \Lambda_{e}^{T}$ and $\widetilde{\mathscr{B}}_{e}=\Lambda_{e} \mathrm{~B}_{e} \Lambda_{e}^{T}$. Hence the characteristic polynomial (4.12) can be expressed in the form

$$
\chi(\lambda)=\frac{\mathbb{I}\left(\Lambda_{e}\right)}{\operatorname{det} \mathbb{I}_{\Lambda_{e}}}\left(\lambda^{4} \mathbb{I}\left(\Lambda_{e}\right)^{-1} \operatorname{det} \mathbb{I}_{\Lambda_{e}}+\lambda^{2}\left(\xi^{2} \rho_{e}^{2}+\left\langle\tilde{\mathscr{B}}_{e}, \mathscr{S}_{e}\right\rangle\right)+\operatorname{det} \widetilde{\mathscr{B}}_{e}\right) .
$$

The energy analysis implies that positive definiteness of $\widetilde{\mathscr{B}}_{e}$ (equivalently, positive semi-definiteness of $\mathscr{B}_{e}$ ) is sufficient to guarantee linear stability of the relative equilibrium, while $(4.18)$ implies that indefiniteness is sufficient to guarantee linear instability. However, negative definiteness of $\widetilde{\mathscr{B}}_{e}$ need not imply instability. For certain classes of tops, linear stability does imply positive semi-definiteness of $\mathscr{B}_{e}$, and hence nonlinear stability. However, there are some classes of tops for which there exists a range of angular velocities at which the top is linearly stable, while $\mathscr{B}_{e}$ is negative semi-definite.

\subsection{Linear stability of sleeping tops}

If the equilibrium configuration $\Lambda_{e}$ is a sleeping top, i.e., if $\mathbf{e}_{3}$ is an eigenvector of $\mathbb{I}_{\Lambda_{e}}$, then the linear stability analysis is substantially simplified. If $\Lambda_{e}$ determines a sleeping top, then $\mathscr{S}_{e}=\mathbb{I}_{2}$, where $\mathbb{I}_{2}$ denotes the restriction of $\mathbb{I}_{\Lambda_{e}}$ to the $\mathbf{e}_{1}-\mathbf{e}_{2}$ plane,

$$
\operatorname{det} \mathbb{I}_{\Lambda_{e}}=\mathbb{I}\left(\Lambda_{e}\right) \operatorname{det} \mathbb{I}_{2}, \quad \text { and } \quad \rho_{e}=\operatorname{tr} \mathbb{I}_{2}-\mathbb{I}\left(\Lambda_{e}\right) \text {. }
$$

In this case, the spatial form (4.18) of the characteristic polynomial simplifies to

$$
\chi(\lambda)=\frac{1}{\operatorname{det} \mathbb{I}_{2}}\left(\lambda^{4} \operatorname{det} \mathbb{I}_{2}+\lambda^{2}\left(\xi^{2} \rho_{e}^{2}+\left\langle\tilde{\mathscr{B}}_{e}, \mathbb{I}_{2}\right\rangle\right)+\operatorname{det} \tilde{\mathscr{B}}_{e}\right)
$$

where $\tilde{\mathscr{B}}_{e}$ is given by (2.17). If $\mathbb{I}_{\Lambda_{e}}=\operatorname{diag}\left[\mathrm{I}_{1}, \mathrm{I}_{2}, \mathbf{I}_{3}\right]$ and $\mathbf{m}_{e}=m \ell \mathbf{e}_{3}$, then (4.20) can be written as

$$
\begin{aligned}
\chi(\lambda)=\frac{1}{\mathrm{I}_{1} \mathrm{I}_{2}}\left(\left(\mathrm{I}_{1}\right.\right. & \left.+\mathrm{I}_{2}-\mathrm{I}_{3}\right)^{2} \xi^{2} \lambda^{2} \\
& \left.+\left(m g \ell-\mathrm{I}_{1} \lambda^{2}+\left(\mathrm{I}_{2}-\mathrm{I}_{3}\right) \xi^{2}\right)\left(m g \ell-\mathrm{I}_{2} \lambda^{2}+\left(\mathrm{I}_{1}-\mathrm{I}_{3}\right) \xi^{2}\right)\right)
\end{aligned}
$$


The characteristic polynomial (4.21) for the linearized equations of motion about an upright sleeping top appears, with slightly different sign conventions, in Routh (1884, section 212).

To simplify the analysis, we introduce the parameters

$$
\begin{aligned}
\delta:=\mathrm{I}_{1}+\mathrm{I}_{2} & \psi:=\frac{2 m g \ell}{\delta} \\
\alpha:=\frac{\mathrm{I}_{1}-\mathrm{I}_{2}}{\delta} & \varphi:=\frac{\delta-\mathrm{I}_{3}}{\delta} .
\end{aligned}
$$

Note that the conditions $0<\mathrm{I}_{i}<\mathrm{I}_{j}+\mathrm{I}_{k}$ for all $i, j$, and $k$ imply $\varphi>0$ and $1-\varphi>|\alpha|$. We first assume that $\psi$ is positive, i.e. that the top is upright; a negative value of $\psi$ indicates that the top is hanging. The characteristic polynomial (4.21) of the linearized equations takes the form

$$
\begin{aligned}
\chi(\lambda)=\frac{4}{1-\alpha^{2}}[ & \varphi^{2} \xi^{2} \lambda^{2}+\frac{1}{4}\left(\psi-(1+\alpha) \lambda^{2}+(2 \varphi-(1+\alpha)) \xi^{2}\right) \\
& \left.\times\left(\psi-(1-\alpha) \lambda^{2}+(2 \varphi-(1-\alpha)) \xi^{2}\right)\right]
\end{aligned}
$$

with respect to these parameters. The eigenvalue transitions are determined by the parameters $\alpha$ and $\varphi$ that characterize the equilibrium inertia tensor. The regions associated to distinct sequences of eigenvalue transitions are indicated in figure 1.

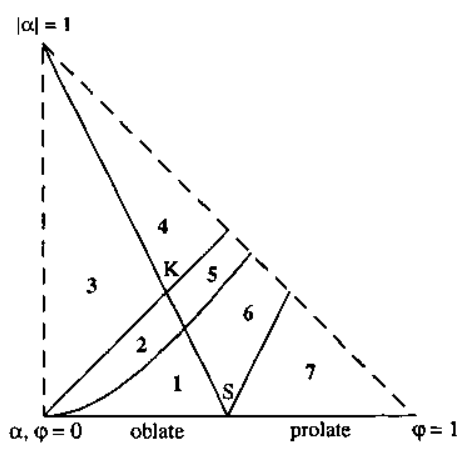

Figure 1. Regions in 'top space' for sleeping tops: the $\varphi-\alpha$ plane.

The broken lines correspond to the limiting configurations in which one of the principal axes has length zero. The vertical broken line represents discs rotating in the plane; the diagonal broken line represents discs rotating about an out-of-plane axis. The points $(1,0)$ and $(0,1)$ correspond to configurations with two principal axes of zero length. The line $\alpha=0$ consists of Lagrange tops; the point $S=(1 / 2,0)$ corresponds to a spherical top and the point $K=(1 / 3,1 / 3)$ is the Kovalevskaya top. Regions 1-3 correspond to tops in rotation about the shortest principal axis (i.e., the axis of greatest moment of inertia). Regions 4-6 correspond to tops rotating about the middle axis and region 7 contains all tops in rotation about the longest axis. Note that only tops in regions 1-3 can ever be stable according to the energy analysis.

Given a top in one of the regions described above, we can consider the possible transitions of the eigenvalues of the linearized system as the angular velocity is 
decreased. The relevant values of $\xi^{2}$ are defined as follows:

$$
\begin{aligned}
\tau_{\mathrm{sf}-\mathrm{f}}^{+} & :=\frac{\psi}{1-2 \varphi+\alpha}=\frac{m g \ell}{\mathrm{I}_{3}-\mathrm{I}_{2}} \\
\tau_{\mathrm{sf}-\mathrm{f}}^{-} & :=\frac{\psi}{1-2 \varphi-\alpha}=\frac{m g \ell}{\mathrm{I}_{3}-\mathrm{I}_{1}} \\
\tau_{\mathrm{f}-\mathrm{s}}^{ \pm} & :=\frac{\psi\left(\varphi-\alpha^{2} \pm \sqrt{\left(1-\alpha^{2}\right)\left(\varphi^{2}-\alpha^{2}\right)}\right)}{\varphi(1-\varphi)^{2}} \\
& =\frac{m g \ell\left(4 \mathrm{I}_{1} \mathrm{I}_{2}-\left(\mathrm{I}_{1}+\mathrm{I}_{2}\right) \mathrm{I}_{3} \pm 2 \sqrt{\mathrm{I}_{1} \mathrm{I}_{2}\left(2 \mathrm{I}_{1}-\mathrm{I}_{3}\right)\left(2 \mathrm{I}_{2}-\mathrm{I}_{3}\right)}\right)}{\mathrm{I}_{3}^{2}\left(\mathrm{I}_{1}+\mathrm{I}_{2}-\mathrm{I}_{3}\right)} .
\end{aligned}
$$

The subscripts sf-f and $\mathrm{f}-\mathrm{s}$ stand for 'superfast-fast' and 'fast-slow'; the terminology 'fast tops' and 'slow tops' is traditional. The values $\tau_{\mathrm{sf}-\mathrm{f}}^{\frac{t}{4}}$ correspond to values of $\xi^{2}$ at which zero double eigenvalues occur. We shall show in section 5 that bifurcations to tilted relative equilibria (moving without spin) occur when $\xi^{2}=\tau_{\mathrm{sf}-\mathrm{f}}^{t}$; tops for which $\tau_{\mathrm{sf}-\mathrm{f}}^{+}$and $\tau_{\mathrm{sf}-\mathrm{f}}^{-}$take on distinct real values undergo a loss of stability when $\xi^{2}=\tau_{\mathrm{sf}-\mathrm{f}}^{+}$ and regain linear stability when $\xi^{2}=\tau_{\mathrm{s} \hat{-}-\mathrm{f}}$. The values $\tau_{\bar{f}-\mathrm{s}}^{ \pm}$correspond to values of $\xi^{2}$ at which non-zero eigenvalues (necessarily double) occur. All upright sleeping tops are linearly unstable for $\xi^{2}<\tau_{\mathrm{f}-\mathrm{s}^{*}}^{+}$

The values $\tau_{\mathrm{s} \tilde{f}-\mathrm{f}}^{t}$ and $\tau_{\tilde{f}-\mathrm{s}}^{ \pm}$are determined as follows: The eigenvalues of the linearized system satisfy

$$
\lambda^{2}=\frac{\varpi \pm 2 \sqrt{\sigma}}{1-\alpha^{2}}
$$

where

$$
\varpi=\psi-\left[(1-\varphi)^{2}+\varphi^{2}-\alpha^{2}\right] \xi^{2}
$$

and

$$
\begin{aligned}
\sigma & =\frac{1}{4} \alpha^{2} w^{2}+\varphi\left(\alpha^{2}-\varphi\right) \psi \xi^{2}+\varphi^{2}(1-\varphi)^{2} \xi^{4} \\
& =\left(\varphi(1-\varphi) \xi^{2}+\frac{1}{2} \alpha \psi\right)^{2}-(1-\alpha)(\varphi+\alpha) \varphi \psi \xi^{2}
\end{aligned}
$$

Non-zero double eigenvalues occur when $\sigma=0, w \neq 0$; zero eigenvalues occur when $\varpi^{2}=4 \sigma$, equivalently when

$$
\operatorname{det} \mathscr{B}_{e}=\delta^{2}\left((1-2 \varphi-\alpha) \xi^{2}-\psi\right)\left((1-2 \varphi+\alpha) \xi^{2}-\psi\right)=0
$$

i.e., when the stability form $\mathscr{B}_{e}$ is singular. The values $\tau_{\mathrm{f}-\mathrm{s}}^{ \pm}$are the roots of the equation $\sigma=0 ; \tau_{\mathrm{sf}-\mathrm{f}}^{+}$are the roots of the equation $\varpi^{2}=4 \sigma$.

For tops lying in region 1 and 2 , all of the above values are positive, and are hence attained by $\xi^{2}$. For tops in regions 3 and 4 , the values $\tau_{f-s}^{ \pm}$are imaginary. We note that $\sigma=(\alpha \psi / 2)^{2} \geqslant 0$ when $\xi=0$ and that

$$
\left|\varphi-\alpha^{2}\right| \geqslant \sqrt{\left(1-\alpha^{2}\right)\left(\varphi^{2}-\alpha^{2}\right)}
$$

implies that both roots of $\sigma=0$ must have the same sign. Hence $\sigma$ is negative, if ever, only for $\xi^{2}$ between the two positive roots. In regions 4,5 and 6 , where $I_{3}$ is neither 
the minimum nor the maximum eigenvalue of $\mathbb{I}_{\Lambda_{e}}, \tau_{\mathrm{sf}-\mathrm{f}}<0$; in these regions $\xi^{2}$ cannot attain the value $\tau_{\mathrm{sf}-f}^{-}$, hence the eigenvalues pass through zero only once, at $\tau_{\mathrm{sf}-\mathrm{f}}^{+}$. In region $7\left(\mathrm{I}_{3}<\mathrm{I}_{2}<\mathrm{I}_{1}\right)$, both $\tau_{\mathrm{sf}-\mathrm{f}}^{+}$and $\tau_{\mathrm{sf}-\mathrm{f}}^{-}$are negative, hence the eigenvalues never pass through the origin. Along the straight line boundaries of the regions $1-7$, the behaviour resembles that of the region to the right of the boundary. Along the curve

$$
\alpha^{2}=\frac{1}{2}\left(1+4 \varphi^{2}-\sqrt{8 \varphi^{2}+1}\right)
$$

where $w$ and $\sigma$ vanish simultaneously for $\xi^{2}=\tau_{f-s}^{-}=\tau_{\mathrm{sf}-\mathrm{f}}^{+}$, an entire window of eigenvalue behaviour shown in figure 2 collapses to a quadruple zero eigenvalue. Note that this curve separates the region of parameter space for which the energy stability criteria and the linear stability criteria agree from the region for which "linear restabilization' can occur. In regions $\mathbf{1 , 6}$, and 7 , the magnetic terms may dominate the second variation $\mathscr{B}_{e}$ for a range of angular velocities for which $\mathscr{B}_{e}$ is negative definite, i.e. it is possible that

$$
\xi^{2} \rho_{e}^{2}>-\left\langle\widetilde{\mathscr{B}}_{e}, \mathbb{I}_{2}\right\rangle>0
$$

Looking at figure 1 , we see that this is possible for relatively oblate or nearly symmetric tops, i.e., tops for which $\varphi$ is sufficiently greater than $|\alpha|$.

The Lagrange tops, which lie along the line $\alpha=0$, are distinct from the asymmetric tops in the following respects: first, $\tau_{\mathrm{sf}-\mathrm{f}}^{+}=\tau_{\mathrm{sf}-\mathrm{f}}^{-}$, hence the 'window of instability' disappears and the eigenvalues of Lagrange tops in region 1 pass through zero only once, remaining on the imaginary axis; second, the transition values $\tau_{f-s}^{+}$simplify to $\tau_{\mathrm{f}-\mathrm{s}}^{+}=\psi /(1-\varphi)^{2}$ and $\tau_{\mathrm{f}-\mathrm{s}}^{-}=0$, hence the collapse of the four distinct imaginary eigenvalues to two double real eigenvalues takes place at $\xi^{2}=0$ and the split to four distinct real eigenvalues does not occur. The difference in behaviour between Lagrange and asymmetric tops is an example of system symmetry breaking.

The eigenvalue transitions associated to each region in figure 1 are given in figures 2-4. The eigenvalue portraits are displayed from left to right in order of decreasing angular velocity. We assume here that $I_{1}>I_{2}$ and, hence, $\tau_{\mathrm{sf}-\mathrm{f}}^{+}>\tau_{\mathrm{sf}-\mathrm{f}}^{-}$; if $I_{2}>I_{1}$, then the appropriate portraits can be obtained by exchanging the labels $\tau_{\mathrm{sf}-\mathrm{f}}^{+}$and $\tau_{\mathrm{sf}}^{-}-\mathrm{f}$. The numbers of the portrait sequences are associated to the numbers of the regions in figure 1; the sequences labeled $\mathbf{L ~} \mathbf{1}$ and $\mathbf{L} 7$ are associated to the Lagrange tops lying on the intersection of the line $\alpha=0$ with regions 1 and 7. The portrait sequence labelled 1-2, respectively 5-6, describes the eigenvalue behaviour of tops lying on the boundary of regions 1 and 2 , respectively 5 and 6 .

The eigenvalue behaviour along the boundaries of the regions is characterized as follows: On the boundaries between regions 1 and 2 and regions 5 and 6, the lower 'superfast-fast' and upper 'fast-slow' transitions collide, as indicated in the eigenvalue transition diagrams. Along the boundary of the regions 2 and 3 , and regions 4 and 5 , i.e. where $|\alpha|=\varphi$, the eigenvalues have the form

$$
\lambda^{2}=\frac{\psi+\xi^{2}(3 \varphi-1)}{1+\varphi} \quad \text { and } \quad \lambda^{2}=\frac{\psi}{1-\varphi}-\xi^{2}
$$

Hence no 'fast-slow' transition occurs along these boundaries and the eigenvalues portraits along the 2-3 boundary (respectively $4-5$ boundary) resemble the portraits for region 3 (respectively 4). Along the 1-6, 2-5, and 3-4 boundaries, the upper 
1.

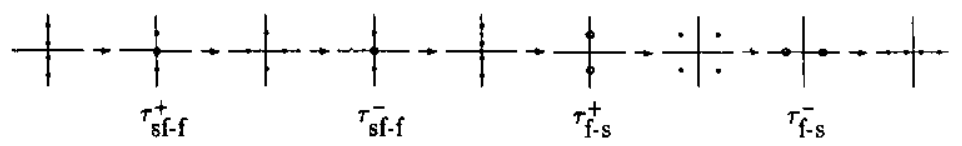

1-2.

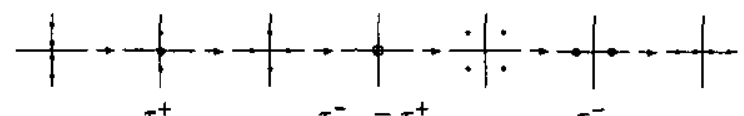

$$
\tau_{\mathrm{sf} f \mathrm{f}}^{+} \quad \tau_{\mathrm{sf}-\mathrm{f}}^{-}=\tau_{\mathrm{f}-\mathrm{s}}^{+} \quad \tau_{\mathrm{f}-\mathrm{s}}^{-}
$$

2.

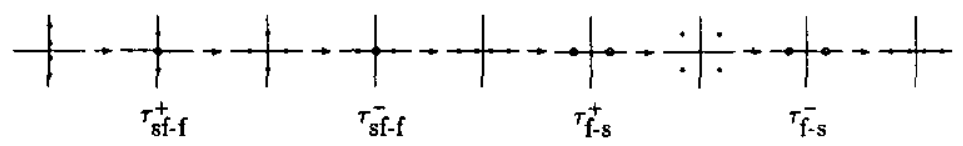

3.

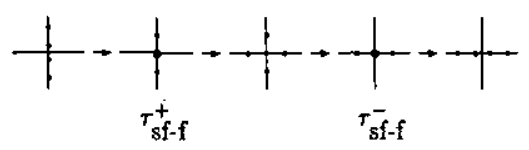

4.

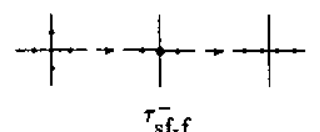

5.

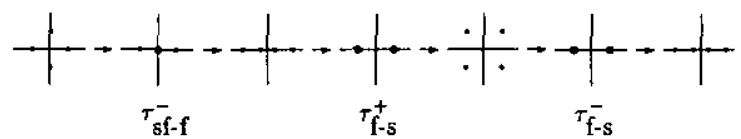

5-6.

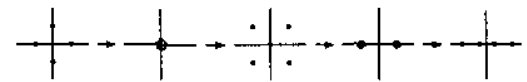

$\tau_{\mathrm{sf}-\mathrm{f}}^{-}=\tau_{\mathrm{f}-\mathrm{s}}^{+} \quad \tau_{\mathrm{f}-\mathrm{s}}^{-}$

6.

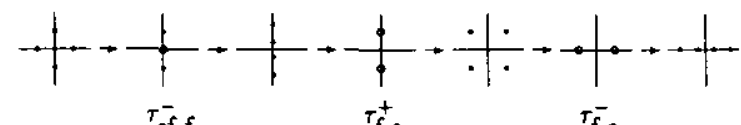

7 .

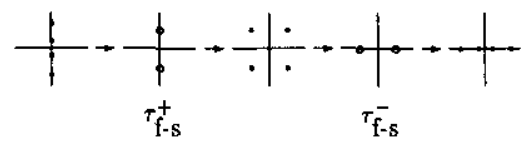

Figure 2. Eigenvalue transitions for upright sleeping tops.

'superfast-fast' transition point $\tau_{\mathrm{sf}-\mathrm{f}}^{+}$occurs at infinite angular velocity; along the 6-7 boundary, the lower 'superfast-fast' transition point $\tau_{\mathrm{sf}-\mathrm{f}}^{-}$occurs at infinite velocity.

Remark: A Hamiltonian-Hopf bifurcation occurs at the $\tau_{f-s}^{+}$transition for tops in regions 1,6 and 7 and for all Lagrange tops. The transition of double conjugate purely imaginary eigenvalues to a conjugate quadruple of eigenvalues with non-zero real part is a characteristic feature of Hamiltonian-Hopf bifurcation, however, some non-degeneracy conditions must be checked to establish the actual occurence of a bifurcation. (See Van der Meer $(1985,1990)$.) We do not carry out that analysis here.

The eigenvalue behaviour of the hanging sleeping top is far simpler than that of the upright top. If $\psi$ is negative, $\sigma$ is positive for all values of $\alpha, \varphi$ and $\xi$. Hence the 'fast-slow' transitions never occur for hanging tops; the eigenvalues always consist of purely real or purely imaginary pairs. Hence the detailed structure of the 'top space' given in figure 1 is no longer relevant; the sequences of eigenvalue transitions are 
L 1 .

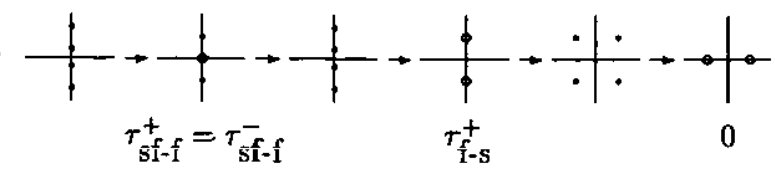

L 7.

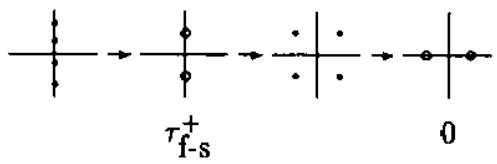

Figure 3. Eigenvalue transitions for upright sleeping Lagrange tops.

determined solely by the relative magnitudes of the principal axes of the inertia tensor. As indicated by the energy analysis of section 2 , hanging sleeping tops for which the axis of rotation $e_{3}$ is the axis of maximal inertia, i.e., for which $I_{3}>I_{1}$ and $I_{3}>I_{2}$, are always stable, with purely imaginary eigenvalues. Tops for which $I_{3}$ is the middle axis, i.e. for which $I_{2}>I_{3}>I_{1}$ or $I_{1}>I_{3}>I_{2}$, are stable only for sufficiently small angular velocities; the eigenvalues undergo a single 'superfast-fast' transition. Finally, if $I_{3}$ is the smallest axis of inertia, then the top is stable for sufficiently large or sufficiently small angular velocities; both of the 'superfast-fast' transitions occur. The eigenvalue transitions are given below; the eigenvalue portraits are displayed from left to right in order of decreasing angular velocity.

$1,2,3, \mathrm{~L} 1$.

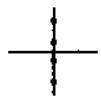

$4,5,6$.

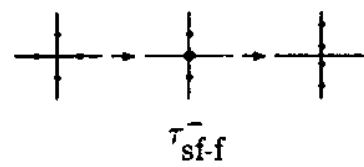

7.

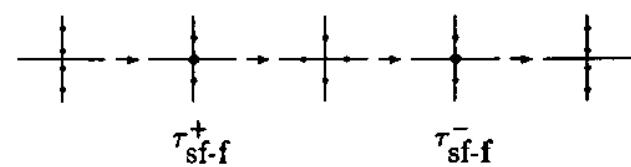

L 7 .

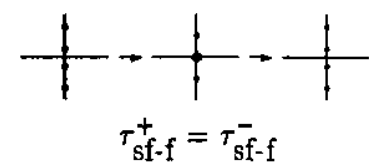

Figure 4. Eigenvalue transitions for hanging sleeping tops.

The possible sequences of eigenvalue behaviour for sleeping tops are summarized in tables 2 and 3. The characteristics of the eigenvalues are indicated by the following conventions, where $\kappa$ and $v$ are distinct non-zero real numbers:

$$
\begin{array}{lll}
\mathrm{II}: \pm \kappa i, \pm v i & \mathrm{DI}: \pm \kappa i \text { (double) } & \mathrm{IZ}: \pm \kappa i, 0 \text { (double) } \\
\mathrm{RR}: \pm \kappa, \pm v & \mathrm{DR}: \pm \kappa \text { (double) } & \mathrm{ZR}: \pm \kappa, 0 \text { (double) } \\
\mathrm{IR}: \pm \kappa i, \pm v & \mathrm{Q}: \pm \kappa \pm v i & \mathrm{ZZ}: 0 \text { (quadruple). }
\end{array}
$$

For example, II indicates two distinct pairs of conjugate imaginary eigenvalues, while DI indicates a conjugate pair of double imaginary eigenvalues, $Q$ indicates a quartet. 
As in the preceding tables, the information is displayed from left to right in order of decreasing velocity; the notation ' $>>$ ' denotes the range of angular velocities between the specific left and right hand values; e.g., the third column describes the eigenvalue behaviour of the top for angular velocities $\xi$ satisfying $\tau_{\mathrm{sf}-\mathrm{f}}^{+}>\xi^{2}>\tau_{\mathrm{sf}-\mathrm{f}^{-}}^{-}$. The first column describes the behaviour of tops for which $\xi^{2}>\tau_{\mathrm{sf}-\mathrm{f}}^{+}$, while the last column decribes tops for which $\tau_{\tilde{f}-s}^{-}>\xi^{2}$. A dash indicates that the eigenvalue behaviour in that velocity range is the same as that in the ajoining ranges.

Table 2. Eigenvalue sequences for an upright sleeping top.

\begin{tabular}{llllllllll}
\hline Region $\backslash \xi^{2}$ & $>$ & $\tau_{\mathrm{sf}-\mathrm{f}}^{+}$ & $>>$ & $\tau_{\mathrm{sf}-\mathrm{f}}^{-}$ & $>>$ & $\tau_{\mathrm{f}-\mathrm{s}}^{+}$ & $>>$ & $\tau_{\mathrm{f}-\mathrm{s}}^{-}$ & $>$ \\
\hline 1 & II & IZ & IR & IZ & II & DI & Q & DR & RR \\
$1-2$ & II & IZ & IR & ZZ & - & ZZ & Q & DR & RR \\
2 & II & IZ & IR & ZR & RR & DR & Q & DR & RR \\
3 & II & IZ & IR & ZR & RR & - & - & - & - \\
4 & - & - & IR & ZR & RR & - & - & - & - \\
5 & - & - & IR & ZR & RR & DR & Q & DR & RR \\
$5-6$ & - & - & IR & ZZ & - & ZZ & Q & DR & RR \\
6 & - & - & IR & IZ & II & DI & Q & DR & RR \\
7 & - & - & - & - & II & DI & Q & DR & RR \\
L 1 & II & IZ & - & IZ & II & DI & Q & DR & - \\
L 7 & - & - & - & - & II & DI & Q & DR & - \\
\hline
\end{tabular}

Table 3. Eigenvalue sequences for a hanging sleeping top.

\begin{tabular}{llllll}
\hline Region $\backslash \zeta^{2}$ & $>$ & $\tau_{\mathrm{sf}-\mathrm{f}}^{+}$ & $>>$ & $\tau_{\mathrm{sf}-\mathrm{f}}^{-}$ & $>$ \\
\hline $1,2,3$, L 1 & - & - & - & - & II \\
$4,5,6$ & - & - & IR & IZ & II \\
7 & II & IZ & IR & IZ & II \\
L 7 & II & IZ & - & IZ & II \\
\hline
\end{tabular}

\subsection{Linear stability of a special class of tilted equilibria}

We can express the characteristic polynomial (4.18) for the simple family of tilted relative equilibria introduced in section 2.3 in terms of the parametrization given in that section. Recall that these are relative equilibria for which the axis of gravity lies in the plane determined by two of the principal axes of the inertia tensor; we restrict our attention to the relative equilibria that satisfy the additional condition that the centre of mass lies along one of these axes, specifically, that the angle $\phi$ determined by (2.39) equals zero. If $\mathbb{I}_{\Lambda_{e}}$ is given by (2.36), then $\mathscr{S}_{e}=\operatorname{diag}\left[\mathrm{I}_{1}, \tau_{s}\right]$, where

$$
\tau_{s}:=\frac{\mathrm{I}_{2} \mathrm{I}_{3}}{\mathrm{I}\left(\Lambda_{e}\right)}=\frac{\mathrm{I}_{2} \mathrm{I}_{3}}{\mathrm{I}_{2} \sin ^{2} \theta+\mathrm{I}_{3} \cos ^{2} \theta}
$$

and $\rho_{e}=\mathrm{I}_{1}-\mathrm{I}_{2}-\mathrm{I}_{3}+2 \tau_{s}$. Since $\widetilde{\mathscr{B}}_{e}=\operatorname{diag}\left[b_{1}, b_{2}\right]$, where $b_{1}$ and $b_{2}$ are given by (2.46) and (2.47), the characteristic polynomial $\chi(\lambda)$ satisfies

$\chi(\lambda)=\frac{1}{\mathrm{I}_{1} \tau_{s}}\left(\lambda^{4} \mathrm{I}_{1} \tau_{s}+\lambda^{2}\left(\xi^{2}\left(\mathrm{I}_{1}-\mathrm{I}_{2}-\mathrm{I}_{3}+2 \tau_{s}\right)^{2}+\mathrm{I}_{1} b_{1}+\tau_{s} b_{2}\right)+b_{1} b_{2}\right)$ 


$$
=\frac{\xi^{4}}{\mathrm{I}_{1} \tau_{s}}\left(\left(\frac{\lambda}{\xi}\right)^{4} \mathrm{I}_{1} \tau_{s}+\left(\frac{\lambda}{\xi}\right)^{2}\left(\left(\mathrm{I}_{1}-\mathrm{I}_{2}-\mathrm{I}_{3}+2 \tau_{s}\right)^{2}+\mathrm{I}_{1} c_{1}+\tau_{s} c_{2}\right)+c_{1} c_{2}\right)
$$

where $c_{i}:=b_{i} / \xi^{2}$. Hence the relative equilibrium is linearly stable if $c_{1} c_{2}>0$ and

$$
\left(\mathrm{I}_{1}-\mathrm{I}_{2}-\mathrm{I}_{3}+2 \tau_{s}\right)^{2}+\mathrm{I}_{1} c_{1}+\tau_{s} c_{2}>2 \sqrt{\tau_{s} \mathrm{I}_{1} c_{1} c_{2}}
$$

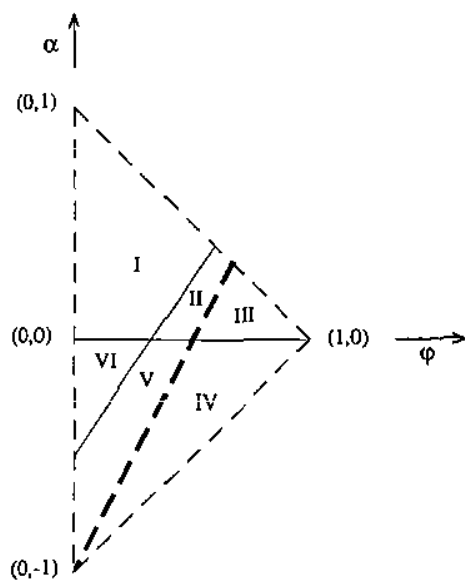

Figure 5. Regions in 'top space' for tilted relative equilibria: the $\varphi-\alpha$ plane.

The bifurcation diagram for these tops is given in figure 5. The boundaries of the regions are determined by the lines $I_{1}=I_{2}, I_{2}=3 I_{3} / 4$, and $I_{2}=I_{3}$. Note that relative equilibria of this class do not exist if $\mathrm{I}_{2}=\mathrm{I}_{3}$. The centre of mass of a relative equilibrium satisfying $I_{3}>I_{2}$ sits above the fixed point of the top, i.e. satisfies $\cos \theta>0$, while the centre of mass of a relative equilibrium satisfying $I_{2}>I_{3}$ sits below the fixed point. Unlike sleeping tops, the stability behaviout of the tilted tops depends on the sign of $\alpha$. As before, the eigenvalue diagrams in figure 6 are displayed form left to right in order of decreasing angular velocity.

I.

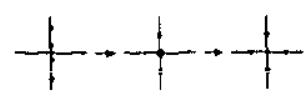

II, IV.

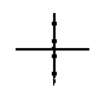

$\alpha=0$.

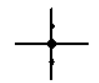

III, V.

VI.

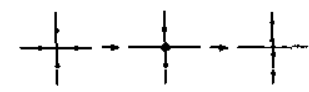

Figure 6. Eigenvalue transitions for tilted tops. 
The relationship between formal and linear stability is much simpler for these tilted tops than for the sleeping tops. We shall show that linear stability corresponds to definiteness (either positive or negative) of the stability matrix $\widetilde{\mathscr{B}}_{e}$, while linear instability corresponds to indefiniteness of $\widetilde{\mathscr{B}}_{e}$. In regions III-VI, $\widetilde{\mathscr{B}}_{e}$ is either positive definite or indefinite; hence our earlier discussion of the characteristic polynomial (4.12) shows that formal stability and linear stability coincide in these regions. In region I, $\widetilde{\mathscr{B}}{ }_{e}$ is negative definite for sufficiently high velocities, as determined by (2.51) and (2.52), and indefinite for lower velocities. The stability form $\widetilde{\mathscr{B}}_{e}$ is negative definite for all relative equilibria with top shape parameters in region II.

In general, negative definiteness of $\widetilde{\mathscr{B}}_{e}$ can correspond to either linear stability or instability. However, we shall show that for this family of relative equilibria, negative definiteness implies linear stability. If we define

$\tau_{\mathrm{t}}:=\left(\mathrm{I}_{1}-\mathrm{I}_{2}-\mathrm{I}_{3}+2 \tau_{\mathrm{s}}\right)^{2}+\mathrm{I}_{1} c_{1}+\tau_{\mathrm{s}} c_{2} \quad$ and $\quad \tau_{\mathrm{d}}:=\tau_{\mathrm{t}}^{2}-4 \mathrm{I}_{1} \tau_{\mathrm{s}} c_{1} c_{2}$

then the linear stability condition is equivalent to the condition that both $\tau_{\mathrm{t}}$ and $\tau_{\mathrm{d}}$ be positive. We shall derive parametrizations of $\cos ^{2} \theta$ and $\mathrm{I}_{i}, i=1,2,3$, which hold whenever $\widetilde{\mathscr{B}}_{e}$ is negative definite and use these parametrizations to show that $\tau_{\mathrm{t}}$ and $\tau_{\mathrm{d}}$ are positive.

The equations $c_{1}=\mathrm{I}_{2}-\mathrm{I}_{1}$ and (2.52) imply that negative definiteness of $\widetilde{\mathscr{B}}_{e}$ holds for relative equilibria with 'top shape parameters' in region I of figure 5 and sufficiently high velocity, i.e. sufficiently small value of $\cos ^{2} \theta$, and for all relative equilibria with 'top shape parameters' in region II of figure 5. We shall derive a parametrization of $\cos ^{2} \theta$ that holds for tops having negative definite second variation; we shall derive distinct parametrizations of the eigenvalues $I_{i}$ of $\mathbb{I}_{\Lambda_{e}}$ for such relative equilibria in regions I and II. Using (2.52), we see that $\mathrm{I}_{3}>\mathrm{I}_{2}$ and $0>b_{2}=\xi^{2} c_{2}$ imply that $\mathrm{I}_{3}>\mathrm{I}_{2}>3\left(\mathrm{I}_{3}-\mathrm{I}_{2}\right) \cos ^{2} \theta$. Hence there exists $\varepsilon_{\mathrm{c}}>0$ such that

$$
\cos ^{2} \theta<\frac{\mathrm{I}_{2}}{3\left(\mathrm{I}_{3}-\mathrm{I}_{2}\right)\left(1+\varepsilon_{\mathrm{c}}\right)} .
$$

In region $I$, the eigenvalues $I_{2}$ and $I_{3}$ satisfy the relationship $3 I_{3} / 4>I_{2}$; in terms of the parameters $\varphi$ and $\alpha$ introduced in section 4.1 , this inequality takes the form $\alpha>(3 \varphi-1) / 2$. Combining this with the general constraint $1-\varphi>\alpha$ derived in section 4.1 , we see that

$$
\alpha=\frac{1}{2}\left(3 \varphi-1+\frac{3-5 \varphi}{1+\varepsilon_{\alpha}}\right)
$$

for some $\varepsilon_{\alpha}>0$. The parameter $\varphi$ attains its maximum value in region $\mathrm{I}$ when $1-\varphi=(3 \varphi-1) / 2$, i.e. when $\varphi=3 / 5$. Since $\varphi$ is always positive, we see that $(\varphi, \alpha) \in$ region I implies that $\varphi=3 /\left(5\left(1+\varepsilon_{\varphi}\right)\right)$ for some $\varepsilon_{\varphi}>0$. Substituting these values into the formulas for $\tau_{\mathrm{t}}$ and $\tau_{\mathrm{d}}$ yields rational functions with positive coefficients; hence, since $\delta, \varepsilon_{\mathrm{c}}, \varepsilon_{\alpha}$, and $\varepsilon_{\varphi}$ are all positive, $\tau_{\mathrm{t}}$ and $\tau_{\mathrm{d}}$ are positive. These substitutions are too unwieldy to be readily carried out by hand; however, they can easily be performed using a symbolic computation language, such as Mathematica $^{\mathrm{TM}}$.

An analogous argument can be applied to region II. The constraint $\mathrm{I}_{3}>\mathrm{I}_{2}>3 \mathrm{I}_{3} / 4$ implies that $(3 \varphi-1) / 2>\alpha>2 \varphi-1$. The parameter $\varphi$ satisfies $2 / 3>\varphi>1 / 3$ in region II. Hence $(\varphi, \alpha) \in$ region II implies that

$$
\varphi=\frac{1}{3}\left(1+\frac{1}{\left(1+\varepsilon_{\varphi}\right)}\right) \quad \text { and } \quad \alpha=2 \varphi-1+\frac{1-\varphi}{2\left(1+\varepsilon_{\alpha}\right)}
$$


for some $\varepsilon_{\varphi}>0, \varepsilon_{\alpha}>0$. Once again, substituting these values into the expressions for $\tau_{\mathrm{t}}$ and $\tau_{\mathrm{d}}$ yields rational functions in $\delta, \varepsilon_{\mathrm{c}}, \varepsilon_{\alpha}$, and $\varepsilon_{\varphi}$ with positive coefficients.

Remark: The Kovalevskaya top: Note that the special class of tilted Kovalevskaya tops discussed in section 2.3, which satisfy $I_{1}=I_{3}=2 I_{2}$ and $I_{r e f} \mathbf{M}=I_{3} \mathbf{M}$, have shape parameters $(\varphi, \alpha)=(1 / 3,1 / 3)$ and hence lie in region $\mathrm{I}$. The analysis given in section 2.3 shows that the stability matrix $\widetilde{\mathscr{B}}_{e}$ is indefinite, and hence the motion is unstable, for $\xi^{2}<2 \sqrt{3} m g \ell / \mathrm{I}_{3}$. For $\xi^{2}>2 \sqrt{3} m g \ell / \mathrm{I}_{3}, \tilde{\mathscr{B}}_{e}$ is negative definite and thus the motion is linearly stable. The linear stability conditions can be stated in terms of the angle $\theta$ as follows: the members of this class of tilted Kovalevskaya tops are linearly stable if $\cos ^{2} \theta<1 / 3$ and unstable if $\cos ^{2} \theta>1 / 3$. This result supplements those of Levi-Civita (1901).

\section{Bifurcations}

A thorough analysis of the possible steady motions of a heavy top is presented in Routh (1884). The heavy top is a sufficiently simple system that it is possible to hypothesize the existence of a particular form of steady motion and then directly determine when, if ever, such motions are possible. For more complicated systems, it can be difficult to 'guess' a priori all possible relative equilibria-abstract results that guarantee the presence (or absence) of particular features can greatly reduce the amount of effort required to locate and characterize families of relative equilibria. Bifurcation theory makes possible the systematic identification of families of relative equilibria; given some initial family of relative equilibria, one can test for the presence of additional families branching from the initial family. An obvious disadvantage of such an approach is its failure to detect isolated families of equilibria; the success of the method depends on the identification of a sufficiently fertile initial branch. (The topological methods of Smale (1970a, b), including the application of Morse theory to the amended potential $V_{\mu}$, are appropriate for the derivation of global information and location of isolated families.) In the presence of symmetries, families of relative equilibria are often organized about some symmetric branch of equilibria: highly symmetric states are likely candidates for stable motions; once such a 'trivial' solution branch has been found, branches of asymmetric relative equilibria can be detected relatively easily by the local bifurcation analysis. In the present treatment, we make use of only the continuous symmetries of the top; this appears to be sufficient to easily obtain the results we seek. However, recent work by Harnad et al (1991) for Hamiltonian systems with both continuous and discrete symmetries, as well the general treatment of bifurcations of symmetric systems described by Golubitsky et al (1988), strongly suggest that the explicit consideration of discrete symmetries can substantially simplify the bifurcation analysis of more complicated systems. Near relative equilibria having positive definite $\widetilde{\mathscr{B}}_{e}$, the Moser-Weinstein theorem guarantees the existence of relative periodic orbits. In fact, one can use discrete symmetries to obtain information on the nature of these periodic orbits using the results of Montaldi et al (1990a, b), as was done for planar coupled rigid bodies by Oh et al (1989). For details of this sort of analysis, see Chong (1991). In the following sections, we show that the tilted steady motions discussed in section 2.3 and section 3 can be found by a simple bifurcation analysis. In particular, we note that the steady precessing motions of the tilted Lagrange top, which combine overall rotation and spin, bifurcate from the steadily rotating sleeping Lagrange top. 


\subsection{Bifurcations from asymmetric sleeping tops}

We first consider bifurcations from the 'sleeping' configurations, i.e. tops for which the axis of gravity is an eigenvector of the equilibrium inertia dyadic, and then show that no bifurcations from the branches of tilted equilibria to relative equilibria occur. In this case, the axis of gravity $e_{3}$ is an eigenvector of the inertia dyadic; we choose the remaining basis vectors so that the inertia dyadic is diagonalized, i.e. $\mathbb{I}_{\Lambda_{\varepsilon}}=\operatorname{diag}\left[\mathrm{I}_{1}, \mathrm{I}_{2}, \mathrm{I}_{3}\right]$.

Recall the family of tilted tops for which the centre of mass lies on the principal axis of inertia with eigenvalue $I_{3}$ that was discussed in section 2.3. If we fix all of the parameters but the angle $\theta$ and the angular velocity $\xi$, then these tops form branches of relative equilibria parameterized by the angle $\theta$ between $\mathbf{m}_{e}$ and $\mathbf{e}_{3}$. As $\theta$ tends towards zero (respectively, $\pi$ ), the family of tops with inertia dyadic (2.36) limit to the sleeping top with inertia dyadic diag $\left[\mathrm{I}_{1}, \mathrm{I}_{2}, \mathrm{I}_{3}\right]$ and angular velocity $\xi^{2}=m g \ell /\left(\mathrm{I}_{3}-\mathrm{I}_{2}\right)$ (respectively $\xi^{2}=m g \ell /\left(\mathrm{I}_{2}-\mathrm{I}_{3}\right)$ ). Analogousiy, there is a one-parameter family of relative equilibria tilted through an angle $\theta$ about the $e_{2}$ axis with angular velocity

$$
\xi^{2}=\frac{m g \ell}{\cos \theta\left(\mathrm{I}_{3}-\mathrm{I}_{1}\right)}
$$

that limits to the sleeping top with angular velocity $\xi^{2}= \pm m g \ell /\left(\mathrm{I}_{3}-\mathrm{I}_{1}\right)$ as $\theta$ approaches 0 or $\pi$.

The sleeping tops with angular velocity $\xi^{2}= \pm m g \ell /\left(\mathrm{I}_{3}-\mathrm{I}_{i}\right)$, where $i=1$ or 2 , lie at the intersections of the curve of sleeping tops, parameterized by $\xi$ with $\theta=0$, with the curves of tilted tops, parameterized by $\theta$, with $\xi$ determined by (2.51). Thus these tops are points of bifurcation. Given the branch of sleeping tops, the existence of the branches of tilted relative equilibria is predicted by the singularity theory techniques described in Golubitsky et al (1985). The underlying principle is the identification of a simple normal form for the function or vector field in question. Possible directions of bifurcation are determined by the Lyapunov-Schmidt reduction procedure; this procedure determines a mapping from the kernel of the linearization of the original mapping into the complement of the image of the linearized mapping. The solutions along the new branch are tangent to the kernel; higher-order correction terms are determined by computing higher-order derivatives of the reduced mapping.

The starting point for the bifurcation analysis in this case is the equation for a relative equilibrium: $D V_{\xi}(\Lambda)=\mathbf{0}$. Since the equilibrium equation is automatically satisfied on the tangent $\mathrm{g} \cdot \Lambda$ to the group orbit, we can restrict our attention to a complement of $g \cdot \Lambda$. Considering (2.33), we see that the second variation $D^{2} V_{\xi}\left(\Lambda_{e}\right)$ changes signature as $\xi^{2}$ passes through the values $m g \ell /\left(\mathrm{I}_{3}-\mathrm{I}_{2}\right)$ and $m g \ell /\left(\mathrm{I}_{3}-\mathrm{I}_{1}\right)$, with null vectors in the direction of the $\mathbf{e}_{1}$ and $\mathbf{e}_{2}$ axes. In the case of a sleeping top with critical angular velocity $\xi_{c}$ satisfying

$$
\xi_{c}^{2}=\frac{m g \ell}{\mathrm{I}_{3}-\mathrm{I}_{2}}
$$

the reduced mapping takes $\operatorname{ker} D^{2} V_{\xi}\left(\Lambda_{e}\right) /\left(g \cdot \Lambda_{e}\right) \approx \operatorname{span}\left\{\mathbf{e}_{1}\right\}$ into itself; hence the reduced bifurcation problem is scalar. As before, let $R_{\theta}$ denote a rotation about $\mathrm{e}_{1}$. Restricting the augmented potential to rotations about $\mathbf{e}_{1}$, we see that

$$
\begin{aligned}
V_{\xi}\left(R_{\theta}\right) & =\frac{1}{4} \xi^{2}\left(\mathrm{I}_{2}+\mathrm{I}_{3}+\left(\mathrm{I}_{3}-\mathrm{I}_{2}\right) \cos 2 \theta\right)-m g \ell \cos \theta \\
& =T_{\xi}(\theta)+\text { higher-order terms }
\end{aligned}
$$


where

$$
T_{\xi}(\theta):=\left(2 \mathrm{I}_{1}-\mathrm{I}_{3}\right) \xi_{c}^{2}+\frac{m g \ell}{2}\left(\frac{3 \theta^{2}}{2}-\frac{\xi^{2}-\xi_{c}^{2}}{\xi_{c}^{2}}\right) \theta^{2}
$$

Clearly, non-zero critical points of $T_{\xi}$ exist if and only if $\xi^{2}>\xi_{c}^{2}$. The general theory guarantees that the behaviour of the full bifurcation problem in a neighbourhood of the possible bifurcation point is described qualitatively by the behaviour of the truncated, reduced system; in particular, the existence of tilted relative equilibria for $\xi^{2}>\xi_{c}^{2}$ follows from the existence of non-zero critical points of $T_{\xi}$. The analysis at the critical velocity $\xi^{2}=m g \ell /\left(\mathrm{I}_{3}-\mathrm{I}_{1}\right)$ is analogous.

We now show that no secondary bifurcations, i.e., bifurcations from the branches of tilted relative equilibria, occur. Âs was previousiy discussed, relative equilibria can be characterized as critical points of the augmented potential $V_{\xi}$; new branches of equilibria must be tangent to a null vector of the second variation of $V_{\xi}$ at the point of bifurcation. On the other hand, relative equilibria are also critical points of the amended potential $V_{\mu}$ and, by the same reasoning, the second variation of $V_{\mu}$ in the direction of the new branch must equal zero. Hence new branches must be tangent to $\operatorname{ker} D \mathbb{I}_{\xi}\left(\Lambda_{e}\right)$. Thus, ignoring the neutral modes associated to the action of the symmetry group, the space of possible directions of bifurcation from a relative equilibrium rotated from the vertical about the $\mathbf{e}_{1}$ axis is given by

$\left(\operatorname{ker} D \mathbb{I}_{\xi}\left(\Lambda_{e}\right)\right) /\left(g_{\mu} \cdot \Lambda_{e}\right)=\operatorname{span}\left\{\mathbb{I}_{\Lambda_{e}} \mathbf{e}_{3}, \mathbf{e}_{3}\right\} / \operatorname{span}\left\{\mathbf{e}_{3}\right\}=\operatorname{span}\left\{\mathbf{e}_{2}\right\}$

since (2.36) implies that

$$
\mathbb{I}_{\Lambda_{e}} \mathbf{e}_{3}=\left(0,\left(I_{2}-I_{3}\right) \cos \theta \sin \theta, I_{2} \sin ^{2} \theta+I_{3} \cos ^{2} \theta\right)
$$

and hence $\operatorname{span}\left\{\mathbf{I}_{\Lambda_{e}} \mathbf{e}_{3}, \mathbf{e}_{3}\right\}=$ span $\left\{\mathbf{e}_{2}, \mathbf{e}_{3}\right\}$. However, (2.47) states that

$$
D^{2} V_{\xi}\left(\Lambda_{e}\right)\left(\mathbf{e}_{2}, \mathbf{e}_{2}\right)=D^{2} V_{\mu}\left(\Lambda_{e}\right)\left(\mathbf{e}_{2}, \mathbf{e}_{2}\right)=\xi^{2}\left(I_{2}-I_{1}\right)>0
$$

so no bifurcation can occur.

We summarize our observations as follows:

Branches of tilted, steadily rotating relative equilibria with angular velocity

$$
\xi^{2}=\frac{m g \ell}{\left(\mathrm{I}_{3}-\mathrm{I}_{i}\right) \cos \theta} \quad i=1,2
$$

where $\theta$ denotes the angle between the axis of gravity and the centre of mass, bifurcate from the branch of sleeping top motions of a heavy top at the angular velocities $\xi^{2}=m g \ell /\left(\mathrm{I}_{3}-\mathrm{I}_{i}\right)$. The tilted equilibria are stable for sufficiently small angles $\theta$ and no secondary bifurcations from these branches occur. 


\subsection{Bifurcations from the sleeping Lagrange top}

Associated to each angular velocity for which the sleeping Lagrange top is stable, there are two one-parameter families of tilted precessing motions that limit to the steadily rotating sleeping top with the specified velocity. These branches, like their asymmetric counterparts, were identified by Routh (1884, sections 102 and 201). The branches that bifurcate from the sleeping Lagrange top are characterized as follows: Assume that the top has rotated out of the vertical by an angle $\theta$; i.e., that the equilibrium inertia dyadic is given by $\mathbb{I}_{\Lambda_{e}}:=R_{\theta} \operatorname{diag}\left[I_{1}, I_{1}, I_{3}\right] R_{\theta}^{T}$, where $R_{\theta}$ is given by (2.37). Replacing $\xi$ with $\xi \lambda$ and $\omega$ with $\xi(\lambda-1)$ in (3.16) and solving for $\lambda$, we find that the top is a relative equilbrium if and only if $\lambda=\lambda(\theta, \xi)$, where

$$
\lambda(\theta, \xi):=\frac{\mathrm{I}_{3} \pm \sqrt{\mathrm{I}_{3}^{2}-4 m g \ell \mathrm{I}_{\theta} / \xi^{2}}}{2 \mathrm{I}_{\theta}}
$$

for $\mathrm{I}_{\theta}:=\mathrm{I}_{3}+\left(\mathrm{I}_{1}-\mathrm{I}_{3}\right) \cos \theta$. The results of section 3 show that the top tipped at an angle $\theta$ with left and right velocities $\pm \xi(\lambda(\theta, \xi), \lambda(\theta, \xi)-1)$ is a stable relative equilibrium. Note that the traditional concept of 'transfer of stability' need not apply to bifurcations of symmetric relative equilibria; the sleeping top is unstable only for angular velocities below those at which bifurcations occur. This is directly related to the non-uniqueness of the variational characterization of relative equilibria, as is discussed below. Since the second variation of the amended potential is always definite along these branches, no secondary bifurcations can occur.

The bifurcations from the sleeping Lagrange top can be detected by singularity theory methods, as was the case for asymmetric tops. The key to the analysis of bifurcations of a symmetric top from the upright 'sleeping' state is the exploitation of the fact that, while left rotations about the axis of gravity cannot be distinguished from right rotations about the axis of symmetry as long as the top is in the upright state, these motions are distinct as soon as the top is tipped out of the vertical. Hence, for a tilted, precessing top, there is a unique pair $(\xi, \omega) \in g$ that yields the specified motion, while for a sleeping Lagrange top, the curve $\xi \mathbf{v}_{\lambda}=\xi(\lambda, \lambda-1)$ in $g$ yields the specified angular velocity $\xi$ for any value of $\lambda$. In section 3.2, we sought conditions under which some value of $\lambda$ could be found for which $V_{\xi \mathbf{v}_{i}}$ had a local minimum at the upright top. The same conditions guarantee that two values of $\lambda$ exist for which $D^{2} V_{\xi \mathbf{v}_{\lambda}}\left(\Lambda_{e}\right)$ is indefinite and thus indicate the possibility of a bifurcation.

This analysis follows a general technique for analysing bifurcations from symmetric relative equilibria, i.e. steady motions for which both the configuration and velocity are fixed by some non-trivial group element, that is described in Lewis (1991b). The following proposition provides sufficient conditions for the bifurcation of an entire surface of relative equilibria from a single symmetric relative equilibrium.

Proposition 1. Assume there is an element $q_{e} \in Q$, algebra elements $\zeta$ and $\alpha \in g$, a differentiable function $f: Q \times \operatorname{span}\{\zeta, \alpha\} \rightarrow \mathbb{R}$, a differentiable one form $\mathbf{h}: Q \rightarrow T^{*} Q$, and a variation $\delta q_{s} \in T_{q_{e}} Q$ satisfying

(i) $D V_{\eta}(q)=f(q, \eta) \mathbf{h}(q)$ for all $q \in Q$ and $\eta \in \operatorname{span}\{\zeta, \alpha\}$

(ii) $\mathbf{h}\left(q_{e}\right)=\mathbf{0}$

(iii) $D\left(\mathbf{h} \cdot \delta q_{s}\right)\left(q_{e}\right) \cdot \delta q_{s} \neq 0$.

Let $I_{\mathrm{b}}$ denote the open set given by

$$
I_{\mathbf{b}}:=\left\{\sigma \in \mathbb{R}: \sigma^{2}\left[\left(\zeta \cdot \delta^{2} \mathbb{I}_{\alpha}\right)^{2}-\left(\alpha \cdot \delta^{2} \mathbb{I}_{\alpha}\right)\left(\zeta \cdot \delta^{2} \mathbb{I}_{\zeta}\right)\right]>-2\left(\alpha \cdot \delta^{2} \mathbb{I}_{\alpha}\right) \delta^{2} V\right\}
$$


where

$$
\delta^{2} V:=D^{2} V\left(q_{e}\right)\left(\delta q_{s}, \delta q_{s}\right) \quad \delta^{2} \mathbb{I}_{\alpha}:=D^{2} \mathbb{I}_{\alpha}\left(q_{e}\right)\left(\delta q_{s}, \delta q_{s}\right) \text {, etc }
$$

Then there is a tubular neighbourhood $\mathscr{W} \subset Q \times \mathbb{R}$ of $\left\{q_{e}\right\} \times I_{\mathrm{b}}$ and a function $\lambda: \mathscr{W} \rightarrow \mathbb{R}$ such that $F L\left(\sigma(\zeta+\lambda(q, \sigma) \alpha)_{Q}(q)\right)$ is a relative equilibrium for all $(q, \sigma) \in \mathscr{W}$.

Condition (iii) is to be interpreted as follows: Let $\mathbf{v}_{s}$ be a vector field satisfying $\mathbf{v}_{s}\left(q_{e}\right)=\delta q_{s}$; the condition that $\mathbf{h}\left(q_{e}\right)=\mathbf{0}$ implies that the first variation at $q_{e}$ of the scalar function $h_{s}(q):=\mathbf{h}(q) \cdot \mathbf{v}_{s}(q)$ does not depend on the choice of $\mathbf{v}_{s}$. We denote the variation $D h_{s}\left(q_{e}\right) \cdot \delta q_{s}$ by $D\left(\mathbf{h} \cdot \delta q_{s}\right)\left(q_{e}\right) \cdot \delta q_{s}$. Proposition 1 is a special case of a more general result in which it is not assumed that $q_{e}$ is a relative equilibrium for all values of $\xi$; in that case, $\xi$ is viewed as a fixed parameter and $\lambda$ can be solved for as a function of $q$ alone. (See Lewis (199̂ 1 b) for the proofs of these results.)

We now apply this result to sleeping Lagrange tops. Let $\mathbb{I}_{\Lambda_{e}}=\operatorname{diag}\left[\mathrm{I}_{1}, \mathrm{I}_{1}, \mathrm{I}_{3}\right]$. Using the $G$ invariance of $V_{(\xi, \omega)}$ and the spatial representation

$$
g \cdot \Lambda=\left\{\mathbf{e}_{3}, \Lambda \mathbf{e}_{3}\right\}
$$

of the tangent space to the group orbit, we see that

$$
D V_{(\xi, \omega)}(\Lambda) \cdot \mathbf{e}_{3} \equiv D V_{(\xi, \omega)}(\Lambda) \cdot \Lambda \mathbf{e}_{3} \equiv 0 .
$$

Hence the one form $\mathbf{h}$ given in the spatial representation by

$$
\mathbf{h}(\Lambda):=\mathbf{e}_{3} \times \Lambda \mathbf{e}_{3}
$$

satisfies $D V_{(\xi, \omega)}(\Lambda)=f(\Lambda,(\xi, \omega)) \mathbf{h}(\Lambda)$ for some function $f: S O(3) \times \mathbb{R}^{2} \rightarrow \mathbb{R}$. Clearly, $\mathbf{h}\left(\Lambda_{e}\right)=\mathbf{0}$ if and only if $\Lambda_{e}$ is a rotation about $\mathbf{e}_{3}$. In this case,

$$
D(\mathbf{h} \cdot \delta \theta)\left(\Lambda_{e}\right) \cdot \delta \theta=\left|\mathbf{e}_{3} \times \delta \theta\right|^{2} .
$$

Hence $D(\mathbf{h} \cdot \delta \theta)\left(\Lambda_{e}\right) \cdot \delta \theta \neq 0$ for $\delta \theta \notin \operatorname{span}\left\{\mathbf{e}_{3}\right\}$.

Let $\delta \theta_{s}$ be an arbitrary unit vector in the $e_{1}-e_{2}$ plane. The second variation of the inertia tensor in the direction of $\delta \theta_{s}$ is

$$
\begin{aligned}
D \mathbb{I}\left(\Lambda_{e}\right)\left(\delta \theta_{s}, \delta \theta_{s}\right) & =\left(\delta \theta_{s} \times \mathbf{e}_{3}\right) \cdot\left(\delta \theta_{s} \times \mathbb{I}_{\Lambda_{e}} \mathbf{e}_{3}\right)\left(\begin{array}{cc}
2 & -1 \\
-1 & 0
\end{array}\right) \\
& -\left(\delta \theta_{s} \times \mathbf{e}_{3}\right) \cdot \mathbb{I}_{\Lambda_{e}}\left(\delta \theta_{s} \times \mathbf{e}_{3}\right)\left(\begin{array}{cc}
2 & 0 \\
0 & 0
\end{array}\right) \\
= & \left(\begin{array}{cc}
2\left(\mathrm{I}_{3}-\mathrm{I}_{1}\right) & -\mathrm{I}_{3} \\
-\mathrm{I}_{3} & 0
\end{array}\right) .
\end{aligned}
$$

We recall the algebra element $\mathbf{v}_{\lambda}=(\lambda, \lambda-1)$ introduced in section $3.2 ; \mathbf{v}_{\lambda}=\xi(\zeta+\lambda \alpha)$ for

$$
\zeta=(0,-1) \quad \text { and } \quad \alpha=(1,1)
$$

The relevant variations of the inertia components are

$$
\alpha \cdot \delta^{2} \mathbb{I}_{\alpha}=-2 \mathrm{I}_{1} \quad \zeta \cdot \delta^{2} \mathbf{I}_{\alpha}=\mathrm{I}_{3} \quad \zeta \cdot \delta^{2} \mathbb{I}_{\zeta}=0 .
$$


The second variation of the potential is $\delta^{2} V=m g \ell$. Hence, using (5.10), we see that

$$
I_{\mathrm{b}}=\left\{\xi: \xi^{2} \mathrm{I}_{3}^{2}>4 \mathrm{I}_{1} m g \ell\right\}
$$

i.e., bifurcations occur from all stable sleeping tops.

In summary:

Tilted spinning, precessing steady motions bifurcate from the branch of sleeping Lagrange tops throughout the range of angular velocities for which the sleeping tops are stable. These tilted motions are always stable.

\section{Geometric derivation of the equations of motion}

In this section, we derive the unreduced (body and spatial) and reduced equations of motions. While these derivations may be of interest to the geometrically oriented reader, a careful reading of this section is by no means necessary for a thorough understanding of the earlier sections of the paper. The derivations of the spatial and body equations (1.32) and (1.33) are included both for the sake of completeness and to introduce the structures necessary for the derivation of the reduced equations. In section 6.2, we attempt to motivate the construction of the shift map (4.1) and prove that the reduced manifold $\mathscr{P}$ defined in (4.5) is, in fact, a Poisson manifold.

\subsection{Derivation of the body and spatial equations of motion}

The symplectic forms on $\mathrm{SO}(3) \times \mathbb{R}^{3}$, thought of as the trivialization of $T^{*} \mathrm{SO}(3)$ via left and right reduction, are

$\omega_{B}(\Lambda, \Pi)\left(\left(\Lambda \widehat{\delta \Theta_{1}}, \delta \Pi_{1}\right),\left(\Lambda \widehat{\delta \Theta}_{2}, \delta \Pi_{2}\right)\right)=\delta \Pi_{2} \cdot \delta \Theta_{1}-\delta \Pi_{1} \cdot \delta \Theta_{2}+\Pi \cdot\left(\delta \Theta_{1} \times \delta \Theta_{2}\right)$

and

$\omega_{S}(\Lambda, \pi)\left(\left(\widehat{\delta \theta}_{1} \Lambda, \delta \pi_{1}\right),\left(\widehat{\delta \theta}_{2} \Lambda, \delta \pi_{2}\right)\right)=\delta \pi_{2} \cdot \delta \theta_{1}-\delta \pi_{1} \cdot \delta \theta_{2}-\pi \cdot\left(\delta \theta_{1} \times \delta \theta_{2}\right)$.

(See Abraham and Marsden (1978), section 4.4 for the derivation of these forms.)

Denoting the Hamiltonian vector field $X_{H}$ defined by $H$ and $\omega_{B}$ by

$$
X_{H}(\Lambda, \Pi)=\left(\Lambda \widehat{X_{\Theta}}(\Lambda, \Pi), X_{\Pi}(\Lambda, \Pi)\right)
$$

we see that $(6.1)$ implies that

$$
\begin{aligned}
\omega_{B}(\Lambda, \Pi)\left(\left(\Lambda \bar{X}_{\Theta}(\Lambda, \Pi), X_{\Pi}(\Lambda, \Pi)\right),(\Lambda \widehat{\delta \Theta}, \delta \Pi)\right) \\
\quad=\delta \Pi \cdot X_{\Theta}(\Lambda, \Pi)-X_{\Pi}(\Lambda, \Pi) \cdot \delta \Theta+\Pi \cdot\left(X_{\Theta}(\Lambda, \Pi) \times \delta \Theta\right) \\
=\left(\Pi \times X_{\Theta}(\Lambda, \Pi)-X_{\Pi}(\Lambda, \Pi)\right) \cdot \delta \Theta+X_{\Theta}(\Lambda, \Pi) \cdot \delta \Pi .
\end{aligned}
$$

If we define the functional derivatives $H_{\Theta}$ and $H_{\Pi} \in \mathbb{R}^{3}$ of a function $H: T^{*} Q \rightarrow \mathbb{R}$ by

$$
D H(\Lambda, \Pi)(\Lambda \widehat{\delta \Theta}, \delta \Pi)=H_{\Theta} \cdot \delta \Theta+H_{\Pi} \cdot \delta \Pi
$$


for all $\delta \Theta, \delta \Pi \in \mathbb{R}^{3}$, then the Hamiltonian vector field $X_{H}$ is determined by requiring that the righthand sides of (6.5) and (6.4) be identical for all $\delta \Theta$ and $\delta \Pi \in \mathbb{R}^{3}$, which yields

$$
X_{H}(\Lambda, \Pi)=\left(\Lambda \widehat{H}_{\Pi}, \Pi \times H_{\Pi}-H_{\Theta}\right)
$$

The differential of the Hamiltonian (1.28) equals

$$
\begin{aligned}
D H(\Lambda, \Pi) \cdot(\Lambda \widehat{\delta \Theta}, \delta \Pi) & \\
= & g(\Lambda \widehat{\delta \Theta})^{T} \mathbf{e}_{3} \cdot \mathbf{M}+\delta \Pi \cdot \mathbf{I}_{\mathrm{ref}}{ }^{-1} \Pi \\
= & -g(\gamma \times \mathbf{M}) \cdot \delta \Theta+\Omega \cdot \delta \Pi .
\end{aligned}
$$

Hence (6.6) yields the Hamiltonian vector field

$$
X_{\Theta}(\Lambda, \Pi)=\Omega \quad \text { and } \quad X_{\Pi}(\Lambda, \Pi)=g \gamma \times \mathbf{M}+\Pi \times \Omega .
$$

The body form (1.32) of the equations of motion follows from the relationship

$$
(\dot{\Lambda}, \dot{\Pi})=X_{H}(\Lambda, \Pi)
$$

The derivation of the spatial form (1.33) of the equations of motion proceeds in a similar manner. If the Hamiltonian vector field associated to the spatial form of the Hamiltonian, given in (1.27), is denoted by $\left(\widehat{Y}_{\theta}(\Lambda, \pi) \Lambda, Y_{\pi}(\Lambda, \pi)\right)$, then

$$
\begin{aligned}
\omega_{S}(\Lambda, \pi)\left(\left(\widehat{Y}_{\theta}(\Lambda, \pi) \Lambda, Y_{\pi}(\Lambda, \pi)\right),(\widehat{\delta \theta} \Lambda, \delta \pi)\right) \\
\quad=-\left(\pi \times Y_{\theta}(\Lambda, \pi)+Y_{\pi}(\Lambda, \pi)\right) \cdot \delta \theta+Y_{\theta}(\Lambda, \pi) \cdot \delta \pi .
\end{aligned}
$$

On the other hand, the first variation of the spatial form (1.27) of the Hamiltonian is

$$
\begin{aligned}
D H(\Lambda, \pi) \cdot( & (\widehat{\delta \theta} \Lambda, \delta \pi) \\
& =\hat{\delta \theta} \mathbf{m} \cdot \mathbf{g}+\delta \pi \cdot \mathbf{I}_{\Lambda}^{-1} \pi-\frac{1}{2} \pi \cdot \mathbb{I}_{\Lambda}^{-1}\left(\widehat{\delta \theta} \mathbb{I}_{\Lambda}-\mathbb{I}_{\Lambda} \widehat{\delta \theta}\right) \mathbb{I}_{\Lambda}^{-1} \pi \\
= & (\mathbf{m} \times \mathbf{g}-\pi \times \omega) \cdot \delta \theta+\omega \cdot \delta \pi .
\end{aligned}
$$

Hence the right hand sides of (6.11) and (6.10) coincide for all variations $\delta \theta$ and $\delta \pi \in \mathbb{R}^{3}$ if and only if the spatial equations of motion (1.33) hold.

For a different derivation of the equations of motion (1.32) and (1.33), obtained by means of the semi-direct product theory, see Marsden, Ratiu and Weinstein (1984).

\subsection{Derivation of the reduced equations}

The shifted reduced equations of motion (4.6) are constructed by Poisson reduction as follows. We first express the symplectic structure on $T^{*} Q$ in terms of the left (body) trivialization, i.e. we identify a configuration-momentum pair $\left(\Lambda, \Pi_{\Lambda}\right) \in T^{*} Q=T^{*}$ $\mathrm{SO}(3)$ with the pair $(\Lambda, \Pi) \in \mathrm{SO}(3) \times \operatorname{so}(3)^{*} \approx \mathrm{SO}(3) \times \mathbb{R}^{3}$ determined by the relationship

$$
\widehat{\Pi}:=\Lambda^{T} \Pi_{\Lambda}
$$

Recall from section 1.3 that in the body representation the Hamiltonian vector field associated to a function $F$ is given by (6.6). Therefore the Poisson bracket $\{F, G\}:=\omega_{B}\left(X_{F}, X_{G}\right)$ of two functions $F, G: T^{*} Q \rightarrow \mathbb{R}$ is given by

$$
\{F, G\}(\Lambda, \Pi)=G_{\Pi} \cdot F_{\Theta}-F_{\Pi} \cdot G_{\Theta}-\Pi \cdot\left(F_{\Pi} \times G_{\Pi}\right) .
$$


We now determine a one-parameter family of induced Poisson brackets on the reduced manifold $\mathscr{P}$. The brackets are parametrized by a momentum $\mu \in \mathrm{g}^{*} \approx \mathbb{R}$ that specifies a 'momentum shift' as follows. Associated to the action of a Lie group $G$ on a manifold $Q$ is a momentum map $\mathbf{J}: T^{*} Q \rightarrow \mathrm{g}^{*}$, where $\mathrm{g}^{*}$ denotes the dual of the Lie algebra of $G$; Noether's theorem guarantees that $\mathbf{J}$ is conserved by the Hamiltonian dynamics associated to a $G$ invariant Hamiltonian $H$ on $T^{*} Q$. The momentum map $\mathbf{J}: T^{*} Q \rightarrow \mathbb{R}$ associated to the $G$ action on $T^{*} Q$ is defined by

$$
\mathbf{J}(\Lambda, \Lambda \widehat{\Pi}) \cdot \xi:=\left\langle\Lambda \widehat{\Pi}, \xi_{Q}(\Lambda)\right\rangle=\xi \Pi \cdot \gamma
$$

where $\gamma=\Lambda^{T} \mathbf{e}_{3}$. The vector field $\zeta: \mathbb{R}^{3} \rightarrow \mathbb{R}^{3}$ given by (4.2) satisfies $\mathbf{J}(\Lambda, \Lambda \widehat{\zeta(\gamma)})=1$. Hence

$$
(\Lambda, \Lambda \widehat{\Pi}) \in \mathbf{J}^{-1}(\mu) \Longleftrightarrow(\Lambda, \Lambda(\widehat{\Pi}-\mu \widehat{\zeta(\gamma)})) \in \mathbf{J}^{-1}(0)
$$

Thus $\Sigma_{\mu}$ maps the momentum level set $\mathbf{J}^{-1}(\mu)$ to the level set $\mathbf{J}^{-1}(0)$. In particular, since (2.2) implies that the body expression for a relative equilibrium in the level $\mathbf{J}^{-1}(\mu)$ is

$$
\boldsymbol{F} L\left(\xi_{Q}(\Lambda)\right)=\boldsymbol{F} L(\Lambda, \xi \Lambda \widehat{\gamma})=\left(\Lambda, \xi \Lambda\left(\widehat{\mathbb{I}_{\mathrm{ref}} \gamma}\right)\right)=(\Lambda, \mu \Lambda \widehat{\zeta(\gamma)})
$$

it follows that relative equilibria are mapped to true equilibria by the appropriate shift map. (The momentum shift is an essential ingredient in the cotangent bundle reduction theorem.)

The induced Poisson bracket of functions on $\mathscr{P}=S^{2} \times \mathbb{R}^{3}$ is defined as follows: A function $f: \mathscr{P} \rightarrow \mathbb{R}$ induces a function $F:=f \circ \Sigma_{\mu}$ on $T^{*} Q$ with functional derivatives

$$
F_{\Theta}=f_{\gamma} \times \gamma-\mu(\nabla \zeta(\gamma) \widehat{\gamma})^{T} f_{\widetilde{\Pi}} \quad \text { and } \quad F_{\Pi}=f_{\widetilde{\Pi}}
$$

where the matrix $\nabla \zeta(\gamma)$ is given by (4.11). Note that $f_{\gamma}$ is defined modulo $\gamma$, since $\gamma \in S^{2}$ implies that all variations $\delta \gamma$ satisfy $\delta \gamma \cdot \gamma=0$. However, the cross product $f_{\gamma} \times \gamma$ is well-defined, so $F_{\Theta}$ is well defined. The Poisson bracket of two such induced functions has the form

$$
\begin{aligned}
\left\{f \circ \Sigma_{\mu}, g \circ \Sigma_{\mu}\right\}(\Lambda, \Pi) & =g_{\widetilde{\Pi}} \cdot\left(f_{\gamma} \times \gamma-\mu(\nabla \zeta \widehat{\gamma})^{T} f_{\widetilde{\Pi}}\right)-f_{\widetilde{\Pi}} \cdot\left(g_{\gamma} \times \gamma-\mu(\nabla \zeta \widehat{\gamma})^{T} g_{\widetilde{\Pi}}\right) \\
& -(\widetilde{\Pi}+\mu \zeta) \cdot\left(f_{\widetilde{\Pi}} \times g_{\widetilde{\Pi}}\right) \\
= & \gamma \cdot\left(g_{\widetilde{\Pi}} \times f_{\gamma}-f_{\widetilde{\Pi}} \times g_{\gamma}\right)-2 \mu f_{\widetilde{\Pi}} \cdot \operatorname{skew}[\nabla \zeta \widehat{\gamma}] g_{\widetilde{\Pi}}-(\widetilde{\Pi}+\mu \zeta) \cdot\left(f_{\widetilde{\Pi}} \times g_{\widetilde{\Pi}}\right)
\end{aligned}
$$

where $(\gamma, \widetilde{\Pi})=\Sigma_{\mu}(\Lambda, \Pi)$. Using the identities

$$
2 \text { skew }[S \widehat{\mathbf{x}}]=(\operatorname{tr} S) \widehat{\mathbf{x}}-\widehat{S \mathbf{x}}
$$

for all symmetric matrices $S$ and all $\mathbf{x} \in \mathbb{R}^{3}$ and

$$
(\nabla \zeta(\gamma)) \gamma=\zeta(\gamma)-2(\zeta(\gamma) \cdot \gamma) \zeta(\gamma)=-\zeta(\gamma)
$$


we obtain

$$
2 \operatorname{skew}[\nabla \zeta(\gamma) \hat{\gamma}]=(\operatorname{tr} \nabla \zeta(\gamma)) \widehat{\gamma}+\widehat{\zeta}(\gamma)=(\operatorname{div} \zeta(\gamma)) \widehat{\gamma}+\widehat{\zeta}(\gamma)
$$

where

$$
\operatorname{div} \zeta(\gamma)=I_{\gamma}^{-1} \operatorname{tr} \mathbb{I}_{\mathrm{ref}}-2|\zeta(\gamma)|^{2}=I_{\gamma}^{-1} r_{\gamma}
$$

is the usual divergence of a vector field on $\mathbb{R}^{3}$. Hence the Poisson bracket $\{,\}_{\mu}$ on $\mathscr{P}$ associated to the momentum $\mu$ is given by

$$
\{f, g\}_{\mu}(\gamma, \widetilde{\Pi})=\gamma \cdot\left(g_{\widetilde{\Pi}} \times f_{\gamma}-f_{\widetilde{\Pi}} \times g_{\gamma}\right)+(\mu(\operatorname{div} \zeta) \gamma-\widetilde{\Pi}) \cdot\left(f_{\widetilde{\Pi}} \times g_{\widetilde{\Pi}}\right) .
$$

Note that the bracket does not depend on the undetermined $\gamma$ component of the partial derivatives $f_{\gamma}$ and $g_{\gamma}$.

The dynamics on the reduced manifold $\mathscr{P}$ are determined by means of the Poisson bracket $\{,\}_{\mu}$ and the induced Hamiltonian $\widetilde{H}_{\mu}$ associated to a specified momentum $\mu$. The total energy $\widetilde{H}_{\mu}$ of the system with 'background momentum' $\mu$, in terms of the reduced variables $(\gamma, \widetilde{\Pi})$, is given by

$$
\widetilde{H}_{\mu}(\gamma, \widetilde{\Pi}):=\frac{1}{2} \widetilde{\Pi} \cdot \mathbb{I}_{\mathrm{ref}}^{-1} \widetilde{\Pi}+\frac{\mu^{2}}{2 I_{\gamma}}+g \gamma \cdot \mathbf{M} .
$$

The reduced Hamiltonian satisfies the condition $\widetilde{H}_{\mu} \circ \Sigma_{\mu}=H$ and has partial derivatives

$$
\left(\widetilde{H}_{\mu}\right)_{\gamma}=g \mathbf{M}-I_{\gamma}^{-2} \mu^{2} \mathbb{I}_{\text {ref }} \gamma \quad(\operatorname{modulo} \gamma)
$$

and

$$
\left(\widetilde{H}_{\mu}\right)_{\widetilde{\Pi}}=\mathbb{I}_{\mathrm{ref}}^{-1} \widetilde{\Pi}=\widetilde{\Omega}
$$

Thus

$$
\begin{gathered}
\dot{f}=\left\{f, H_{\mu}\right\}_{\mu}=\gamma \cdot\left(\tilde{\mathbf{\Omega}} \times f_{\gamma}-f_{\widetilde{\boldsymbol{\Pi}}} \times\left(g \mathbf{M}-2 I_{\gamma}^{-2} \mu^{2} \Pi_{\mathrm{ref}} \gamma\right)\right)+(\mu(\operatorname{div} \zeta) \gamma-\tilde{\Pi}) \cdot\left(f_{\widetilde{\boldsymbol{\Pi}}} \times \widetilde{\mathbf{\Omega}}\right) \\
=f_{\gamma} \cdot(\gamma \times \widetilde{\mathbf{\Omega}})+f_{\widetilde{\Pi}} \cdot\left(\gamma \times\left(g \mathbf{M}-I_{\gamma}^{-2} \mu^{2} \Pi_{\mathrm{ref}} \gamma-\mu(\operatorname{div} \zeta) \widetilde{\Omega}\right)+\widetilde{\Pi} \times \widetilde{\mathbf{\Omega}}\right) .
\end{gathered}
$$

Setting $\xi=I_{\gamma}^{-1} \mu$ in (6.27), we obtain the reduced equations of motion (4.6).

\section{Acknowledgments}

We thank the Mathematical Sciences Institute at Cornell University, where this work was initiated, for its support during the program on Hamiltonian dynamics in the fall of 1989. DL thanks the Institute for Mathematics and its Applications, at the University of Minnesota, Minneapolis, for its support during much of this work and Wolfram Research Incorporated for generously providing Mathematica ${ }^{\mathrm{TM}}$. We thank $\mathrm{J}$ Maddocks for a valuable exchange of ideas which motivated us to analyse in detail relative equilibria other than sleeping tops. 


\section{Appendix. The reduced energy-momentum method}

\section{A1. Glossary: Simple mechanical systems with symmetry}

$Q \quad$ Configuration space, with elements denoted by $q \in Q$

$P=T^{*} Q \quad$ Phase space. Points $z=(q, p) \in P$ are configurations and momenta

$G \quad$ Lie group acting freely on $Q$ on the left; the action of $G$ on $P$ is symplectic, obtained by cotangent lifts

$g \quad$ Lie algebra of $G$, with elements denoted by $\eta \in g$

exp Exponential map exp $: g \rightarrow G$ satisfying $\left.\frac{\mathrm{d}}{\mathrm{d} \epsilon}\right|_{\epsilon=0} \exp (\epsilon \xi)=\xi$

$g^{*} \quad$ Dual of $g$, with elements denoted by $\mu \in g^{*}$

$\operatorname{Ad}_{g} \quad$ Adjoint action of $G$ on $g ; \operatorname{Ad}_{g} \eta=\left.\frac{d}{d \epsilon}\right|_{\epsilon=0} g(\exp (\epsilon \eta)) g^{-1}=[v, \eta]$

$\operatorname{Ad}_{g}^{*} \quad$ Coadjoint action of $G$ on $g^{*} ;\left(\operatorname{Ad}_{g}^{*} \mu\right) \cdot \eta=\mu \cdot \operatorname{Ad}_{g} \eta$

$\operatorname{ad}_{y}$

$\mathrm{ad}_{v}^{*}$

Infinitesimal adjoint action of $g$ on $g ; \operatorname{ad}_{v} \eta=\left.\frac{\mathrm{d}}{\mathrm{d} \epsilon}\right|_{\epsilon=0} \operatorname{Ad}_{(\exp (\epsilon v))} \eta$

$G_{\mu}$

Infinitesimal coadjoint action of $g$ on $g^{*} ;\left(\operatorname{ad}_{v}^{*} \mu\right) \cdot \eta=\mu \cdot \operatorname{ad}_{v} \eta$

Isotropy subgroup of $\mu \in \mathrm{g}^{*}$ under the coadjoint action

$G_{\mu}:=\left\{h \in G: \operatorname{Ad}_{h}^{*} \mu=\mu\right\}$

$g_{\mu} \quad$ Isotropy subalgebra of $\mu \in \mathrm{g}^{*}$ under the infinitesimal coadjoint action $g_{\mu}:=\left\{\eta \in g: \operatorname{ad}_{\eta}^{*} \mu=\mathbf{0}\right\}$

$\eta_{Q} \quad$ Infinitesimal generator $\eta_{Q}(q)=\left.\frac{\mathrm{d}}{\mathrm{d} \epsilon}\right|_{\epsilon=0} \exp (\epsilon \eta) \cdot q$

$g_{\mu} \cdot q_{e} \quad$ Tangent to the orbit of the isotropy subgroup $G_{\mu}$

$\mathrm{g}_{\mu} \cdot q_{e}=\left\{\eta_{Q}\left(q_{e}\right): \eta \in g_{\mu}\right\} \subset T_{q_{e}} Q$

J Momentum map $\mathbf{J}: P \rightarrow \mathrm{g}^{*} ; \mathbf{J}(q, p) \cdot \eta=\left\langle p, \eta_{Q}(q)\right\rangle$

$\langle\langle\cdot, \cdot\rangle\rangle \quad G$ invariant Riemannian metric on $Q$

II Locked inertia tensor II : $Q \rightarrow L\left(\mathrm{~g}, \mathrm{~g}^{*}\right) ;(\mathbb{I}(q) \zeta) \cdot \eta=\left\langle\left\langle\eta_{Q}(q), \zeta_{Q}(q)\right\rangle\right\rangle$

$\mathbb{I}_{\xi} \quad$ Locked momentum map $\mathbb{I}_{\xi}: Q \rightarrow g^{*} ; \mathbb{I}_{\xi}(q)=\mathbb{I}(q) \xi$

$\boldsymbol{F} L \quad$ Legendre transformation $\boldsymbol{F} L: T Q \rightarrow T^{*} Q ;\left\langle\boldsymbol{F} L\left(v_{q}\right), w_{q}\right\rangle=\left\langle\left\langle v_{q}, w_{q}\right\rangle\right\rangle$

$V \quad G$-invariant potential energy $V: Q \rightarrow \mathbb{R}$

$H \quad$ Hamiltonian function $H: P \rightarrow \mathbb{R} ; H(q, p)=\frac{1}{2}\left\|\boldsymbol{F} L^{-1}(p)\right\|^{2}+V(q)$

$V_{\xi} \quad$ Augmented potential $V_{\xi}: Q \rightarrow \mathbb{R} ; V_{\xi}(q)=V(q)-\frac{1}{2} \xi \cdot \mathbb{I}(q) \xi$

$V_{\mu} \quad$ Amended potential $V_{\mu}: Q \rightarrow \mathbb{R} ; V_{\mu}(q)=V(q)+\frac{1}{2} \mu \cdot \mathbb{I}^{-1}(q) \mu$

$\mathscr{B}_{e} \quad$ Stability two form $\mathscr{B}_{e}: T_{q_{e}} Q \times T_{q_{e}} Q \rightarrow \mathbb{R}$

$\mathscr{B}_{e}(\delta q, \Delta q)=D^{2} V_{\xi}\left(q_{e}\right)(\delta q, \Delta q)+\left(D \mathbb{H}_{\xi}\left(q_{e}\right) \cdot \delta q\right) \cdot \mathbb{I}\left(q_{e}\right)^{-1}\left(D \mathbb{H}_{\xi}\left(q_{e}\right) \cdot \Delta q\right)$

$\mathscr{A} \quad$ Generalized Arnold form $\mathscr{A}: \mathbf{g} \times \mathbf{g} \rightarrow \mathbb{R}$

$\mathscr{A}(\zeta, \eta)=\operatorname{ad}_{\zeta}^{*} \mu \cdot\left(\mathbb{I}\left(q_{e}\right)^{-1} \operatorname{ad}_{\eta}^{*} \mu+\operatorname{ad}_{\eta}\left(\mathbb{I}\left(q_{e}\right)^{-1} \mu\right)\right)$

$\mathcal{Q}_{\text {int }} \quad$ Space of 'internal' variations

$\mathcal{Q}_{\text {int }}=\left\{\delta q \in T_{q_{e}} Q: \mathbb{I}\left(q_{e}\right)^{-1}\left(D \mathbb{\Pi}_{\xi}\left(q_{e}\right) \cdot \delta q\right) \in g_{\mu}\right\}$

A2. Summary of the method

An element $z_{e} \in P$ is a relative equilibrium if the trajectory $z_{\mathrm{t}}$ passing through $z_{e}$ determined by the Hamiltonian dynamics satisfies

$$
z_{\mathrm{t}}=\exp (t \xi) \cdot z_{e}
$$

for some $\xi \in g$. Since the $G$ action is free, $z_{e} \in T_{q_{e}^{*}}^{*} Q$ satisfies the above equation if and only if $z_{e}:=F L\left(\xi_{Q}\left(q_{e}\right)\right)$ and $q_{e}$ is a critical point of $V_{\xi}$. The equilibrium configuration $q_{e}$ is also a critical point of $V_{\mu}$ for $\mu:=\mathbb{I}_{\xi}\left(q_{e}\right)$. 
The relative equilibrium $z_{e}$ is (formally) stable modulo $G_{\mu}$ if $D^{2} V_{\mu}\left(q_{e}\right)=\mathscr{B}_{e}$ is positive semi-definite, with kernel $g_{\mu} \cdot q_{e}$. (If the configuration space $Q$ is isomorphic to the symmetry group $G$, e.g., in the free rigid body, then the relative equilibrium is (formally) stable if $\mathscr{B}_{e}$ is either positive or negative definite.) The stability conditions can be decomposed into 'rigid' and 'internal' conditions as follows. The stability form $\mathscr{B}_{e}$ is positive semi-definite with kernel $\mathrm{g}_{\mu} \cdot q_{e}$ if and only if the restriction of $\mathscr{B}_{e}$ to the space $\mathscr{Q}_{\text {int }}$ of 'internal' variations is positive semi-definite with kernel $g_{\mu} \cdot q_{e}$ and the generalized Ârnold form $\mathscr{A}$ is positive semi-definite with kernel $g_{\mu}$. If the symmetry group $G$ is abelian, as in the case of the heavy top, then $g=g_{\mu}$ for all values of $\mu$; hence, in this case, the 'rigid' stability conditions are trivially satisfied and $\mathscr{Q}_{\text {int }}=T_{q_{e}} Q$.

\section{References}

Abraham R and Marsden J E 1978 Foundations of Mechanics second edn (Reading, MA: Benjamin)

Arnold V I 1966 Sur la géométrie différentielle des groupes de Lie de dimension infinie et ses applications à l'hydrodynamique des fluides parfaits Ann. Inst. Fourier XVI 319-61

- 1969 The Hamiltonian nature of the Euler equations in the dynamics of a rigid body and an ideal fluid Usp. Mat. Nauk, 24 225-6

- 1988 Encyclopaedia of Mathematical Sciences Volume 3; Dynamical Systems 11 (New York: Springer)

- Mathematical Methods of Classical Mechanics 2nd edn (New York: Springer)

Chong W D 1991 Quasi-periodic and periodic motions of a heavy rigid body about a fixed point PhD Thesis SUNY, Buffalo

Gantmacher F R 1989 Matrix Theory vol II (New York: Chelsea)

Golubitsky M and Schaeffer D 1985 Singularities and Groups in Bifurcation Theory vol I (New York: Springer)

Golubitsky M, Schaeffer D and Stewart I 1988 Singularities and Groups in Bifurcation Theory vol II (New York: Springer)

Harnad J, Hurtubise J and Marsden J E 1991 Reduction of Hamiltonian systems with discrete symmetries (preprint)

Holm D, Marsden J, Ratiu T and Weinstein A 1985 Nonlinear stability of fluid and plasma equilibria Physics Reports 123 (1 \& 2) 1-116; See also 1983 Physics Lett. A 98 15-21

Iacob A 1971 Invariant manifolds in the motion of a rigid body about a fixed point Rev. Roum. Math. Pures Appl. 16 1497-521

Katok S 1972 Bifurcation sets and integral manifolds in the problem of the motion of a heavy rigid body Usp. Mat. Nauk 27 (2) (Russian)

Klein F and Sommerfeld A 1910 Theorie des Kriesels (Leipzig: Teubner)

Levi-Civita T 1901 Sui moti stazionari di un corpo rigido nel caso Kowalesky Atti Accad. Lincej, Rendiconti, Classe di scienze fisiche, matematiche e naturali 10 I 338-46; II 429-34; III 461-6

Lewis D 1991a Lagrangian block diagonalization J. Dynam. Diff. Eg. 3 619-59

- $1991 \mathrm{~b}$ Bifurcations of liquid drops (preprint)

Lewis D and Simo J C 1990 Nonlinear stability of rotating pseudo-rigid bodies Proc. R. Soc. A 427 281-319

Maddocks J H 1989 On the stability of relative equilibria (preprint)

Marsder J, Ratiu T and Weinstein A 1984 Reduction and Hamiltonian structures on duals of semidirect product Lie algebras Cont. Math. 28 55-100

Montaldi J, Roberts M and Stewart I 1990a Existence of nonlinear normal modes of symmetric Hamiltonian systems Nonlinearity $3695-730$

- 1990b Stability of nonlinear normal mods of symmetric Hamiltonian systems Nonlinearity 3 731-77

Oh Y-G, Sreenath N, Krishnaprasad P S and Marsden J E 1989 The dynamics of coupled planar rigid bodies. II Bifurcations, periodic solutions, and chaos J. Dyn. Diff. Eqn. 3 No $1269-98$

Riemann B 1860 Untersuchungen über die Bewegung eines flüssigen gleichartigen Ellipsoides Abh. d. Königl. Gesell. der Wis. zu Göttingen 9 3-36

Routh E J 1882 Elementary rigid dynamics (London: McMillan)

1884 Advanced rigid dynamics (London: McMillan)

Simo J C, Lewis D and Marsden J E 1991a The stability of relative equilibria. Part I: The reduced energy-momentum method The Archive for Rational Mechanics and Analysis 115 15-59 
Simo J C, Posbergh T A and Marsden J E 1990 Stability of coupled rigid body and geometrically exact rods: Block diagonalization and the Energy-Momentum method Phys. Rep. 193 280-360

_ _ 1991b The stability of relative equilibria. Part II: Application to nonlinear elasticity The Archive for Rational Mechanics and Analysis 115 61-100

Smale S 1970a Topology and Mechanics I Inventiones mathematicae 10 305-31

1970b Topology and Mechanics II Inventiones mathematicae 11 45-64

Tatarinov Ya V 1974 Portraits of the classical integrals of the problem of rotation of a rigid body about a fixed point Vestn. Mosk. Univ., Series 129 No. $699-105$ (Russian)

Van der Meer J C 1985 The Hamiltonian-Hopf bifurcation PhD Thesis Utrecht

1990 Hamiltonian-Hopf bifurcation with symmetry Nonlinearity 3 1041-56 